\title{
Drinfeld-Moduln und elliptische Garben
}

\author{
Dissertation \\ zur Erlangung des Doktorgrades \\ der Mathematisch-Naturwissenschaftlichen Fakultäten \\ der Georg-August-Universität zu Göttingen
}

vorgelegt von

\section{Stefan Wiedmann}

aus

Ochsenhausen

Göttingen 2004 
D7

Referent: Prof. Dr. Ulrich Stuhler

Korreferent: Prof. Dr. Yuri Tschinkel

Tag der mündlichen Prüfung: 28. 10. 2004 


\section{Inhaltsverzeichnis}

1 Einleitung 5

2 Algebraische Kurven und Vektorbündel 9

2.1 Algebraische Hilfsmittel . . . . . . . . . . . . . . . . . 9 9

2.2 Geometrische Hilfsmittel . . . . . . . . . . . . . . . . 12

2.3 Glatte und irreduzible algebraische Kurven . . . . . . . . . . 14

2.4 Projektive algebraische Kurven . . . . . . . . . . . . . 15

2.5 Vektorbündel . . . . . . . . . . . . . . . . . . . . 19

2.6 Kohomologie und Basiswechsel . . . . . . . . . . . . 20

2.7 Twists von Vektorbündeln auf parametrisierten Kurven . . . 24

2.8 Konstruktion von Vektorbündeln auf $X \times S \ldots \ldots$

2.9 Frobenius-Twist von Garben . . . . . . . . . . . . . 31

3 Drinfeld-Moduln und ihre Vektorbündel 34

3.1 Drinfeld-Moduln . . . . . . . . . . . . . . . 34

$3.2 \quad \mathbf{R} \otimes \mathbf{A}$-Modulstruktur auf $\mathbf{R}\{\tau\} \ldots \ldots \ldots . \ldots . \ldots . . \ldots 37$

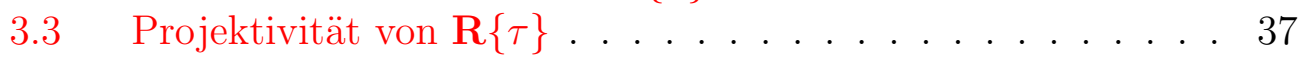

3.3.1 $\mathbf{R}=\mathbf{L}$ ist ein Körper und $\mathbf{A}=\mathbb{F}_{\mathbf{q}}[\mathbf{T}] \ldots \ldots . . .38$

3.3.2 $\mathbf{R}=\mathbf{L}$ ist ein Körper . . . . . . . . . . . . . . 38

3.3.3 $\mathbf{R}$ ist ein reduzierter, noetherscher Ring . . . . . . . . 39

3.3.4 $\mathbf{R}$ ist ein noetherscher Ring . . . . . . . . . . . . 39

3.3.5 $\mathbf{R}$ ist ein beliebiger, kommutativer Ring . . . . . . . 39

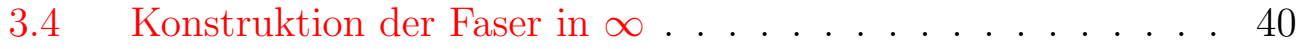

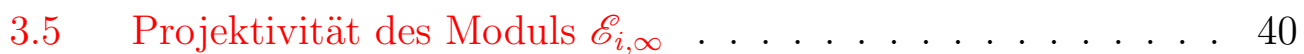

3.5.1 $\mathbf{R}=\mathbf{L}$ ist ein Körper . . . . . . . . . . . . . . 43

3.5.2 $\mathbf{R}$ ist ein reduzierter, noetherscher Ring . . . . . . . . . 44

3.5.3 $\mathrm{R}$ ist ein noetherscher Ring . . . . . . . . . . . . 44

3.5.4 $\mathrm{R}$ ist ein beliebiger Ring . . . . . . . . . . . . 45

3.6 Konstruktion des Vektorbündels . . . . . . . . . . . . 46

3.7 Alternative Konstruktion . . . . . . . . . . . . . . 49

3.8 Konstruktion über einem beliebigen Basisschema . . . . . . . 50

4 Vektorbündel von allgemeinem Typ $\mathbf{5 2}$

4.1 Definition der Vektorbündel von allgemeinem Typ . . . . . . 52

4.2 Drinfelds Theorem . . . . . . . . . . . . . . 52

4.3 Kategorie I $\Longrightarrow$ Kategorie II . . . . . . . . . . . . . . . 53

4.4 Kategorie II $\Longrightarrow$ Kategorie I . . . . . . . . . . . . . . . . . 58

4.4.1 $\mathbf{R}=\mathbf{L}$ ein Körper und $\mathbf{A}=\mathbb{F}_{\mathbf{q}}[\mathbf{T}] \ldots \ldots 1$

4.4.2 $\mathbf{R}=\mathbf{L}$ ein Körper . . . . . . . . . . . . . 61 
4.4.3 R reduzierter, noetherscher Ring . . . . . . . . . . 62

4.4.4 R beliebiger Ring . . . . . . . . . . . . . . . 63

4.5 Kategorienäquivalenz . . . . . . . . . . . . . . 67

4.6 Drinfelds Theorem, erweiterte Fassung . . . . . . . . . . 68

5 Elliptische Garben $\quad 71$

5.1 Definition von elliptischen Garben . . . . . . . . . . . . 71

5.2 Eigenschaften . . . . . . . . . . . . . . . . 72

5.3 Drinfeld-Moduln und elliptische Garben . . . . . . . . . . . . 74

5.3.1 Elliptische Garben $\Longrightarrow$ Drinfeld-Moduln . . . . . . . . 74

5.3.2 Konstruktion eines Drinfeld-Modul . . . . . . . . . 77

5.3.3 Drinfeld-Moduln $\Longrightarrow$ Elliptische Garben . . . . . . . 78

5.4 Beliebiges Basisschema . . . . . . . . . . . . . 79

5.4.1 Drinfeld-Moduln $\Longrightarrow$ Elliptische Garben . . . . . . . . 80

5.4.2 Elliptische Garben $\Longrightarrow$ Drinfeld-Moduln . . . . . . . . 81

5.4.3 Drinfeld-Moduln $\Longrightarrow$ Kategorie II' . . . . . . . . . . . 82

5.4.4 Kategorienäquivalenz . . . . . . . . . . . . 83

6 Teilungspunkte und Level-Strukturen $\quad 84$

6.1 Teilungspunkte und Level-Strukturen von Drinfeld-Moduln . . . . . . . . . . . . . . . . . 84

$6.2 \quad F$-Garben . . . . . . . . . . . . . . . . 87

6.3 Teilungspunkte und Level-Strukturen elliptischer Garben . . 89

6.4 Vergleich der beiden Konzepte . . . . . . . . . . . . . . . . . 91

7 Ausblick $\quad 93$

7.1 D-elliptische Garben . . . . . . . . . . . . . . . 93

7.2 Level-Strukturen . . . . . . . . . . . . . . . . . 94

7.3 Level-Strukturen in der Charakteristik . . . . . . . . . . . 95

$\begin{array}{lll}8 & \text { Literaturverzeichnis } & 97\end{array}$ 


\section{$1 \quad$ Einleitung}

Zum besseren Verständnis des Inhalts der Arbeit möchte der Autor zunächst eine Einführung in die Theorie der Drinfeld-Moduln geben.

\section{Drinfeld-Moduln}

Sei $X$ eine geometrisch zusammenhängende, glatte, algebraische Kurve über dem endlichen Körper $\mathbb{F}_{q}, \infty \in X$ ein abgeschlossener Punkt und $A:=$ $\Gamma\left(X \backslash \infty, \mathcal{O}_{X}\right)$ der Ring der regulären Funktionen außerhalb des Punktes $\infty$. Der Ring $A$ ist in diesem Fall ein Dedekindring. Für ein geeignetes Element $T \in A$ ist $A$ eine endliche $\mathbb{F}_{q}[T]$-Algebra und der ganze Abschluss von $\mathbb{F}_{q}[T]$ in $F$, dem Funktionenkörper der Kurve $X$.

In der klassischen Theorie der algebraischen Zahlkörper (Charakteristik 0) entspricht $\mathbb{F}_{q}[T]$ den ganzen Zahlen $\mathbb{Z}$ und der Ring $A$ entspricht einem Ring ganzer Größen eines algebraischen Zahlkörpers.

Bei der Untersuchung arithmetischer Fragestellungen hat es sich als erfolgreiche Strategie erwiesen, den zahlentheoretischen Objekten algebraisch-geometrische Objekte zuzuordnen. Eine dieser Möglichkeiten ist die Betrachtung elliptischer Kurven, also glatter, geometrisch irreduzibler Kurven von Geschlecht 1 über einem Basisobjekt (z.B. $\mathbb{Q}$ oder $\mathbb{C}$ ). Die hervorragendste Eigenschaft von elliptischen Kurven ist die Struktur einer abelschen Gruppe auf den Punkten der elliptischen Kurve. Die Gruppenstruktur wird hierbei durch algebraische Gleichungen beschrieben, die über dem Basisobjekt definiert sind; wir erhalten ein abelsches Gruppenschema.

Die Untersuchung der Gruppenstruktur und ihren Beziehungen zu Basiswechseln ist außerordentlich reichhaltig. Als Stichworte seien zum Beispiel $N$-Teilungspunkte, $\zeta$-Funktionen, Tate-Moduln, Galois-Darstellungen, Langlands-Korrespondenzen, Modulräume genannt.

Mit Hilfe der Theorie der Weierstrass'schen §-Funktion erhalten wir eine Korrespondenz zwischen den elliptischer Kurven über $\mathbb{C}$ und diskreten $\mathbb{Z}$ Gittern von Rang 2 in $\mathbb{C}$ modulo Multiplikation mit einem Element aus $\mathbb{C}^{\times}$. Im Funktionenkörper-Fall gibt es eine analoge Korrespondenz:

Sei $\mathbb{C}_{\infty}$ die Komplettierung eines algebraischen Abschlusses der Komplettierung von $F$ im Punkt $\infty$. Ein $A$-Gitter von Rang $d$ in $\mathbb{C}_{\infty}$ ist ein endlich erzeugter, diskreter $A$-Untermodul in $\mathbb{C}_{\infty}$. Können wir die Äquivalenzklassen dieser Gitter modulo der $\mathbb{C}_{\infty}^{\times}$-Aktion als Modulraum geeigneter algebraischgeometrischer Objekte beschreiben?

Die Fragestellung wurde von V.G. Drinfeld in seinen Artikeln Elliptic Modules I/II (siehe [Dri76] und [Dri77]) positiv beantwortet. Er führt in seinen Artikeln elliptische Moduln ein. Diese wurden später nach ihrem Erfinder in 
Drinfeld-Moduln umbenannt.

Ein Drinfeld-Modul (über $\mathbb{C}_{\infty}$ ) ist ein nicht trivialer Ringhomomorphismus $e$ von $A$ in den Ring der algebraischen, $\mathbb{F}_{q}$-linearen Endomorphismen der additiven Gruppe $\mathbb{G}_{a / \mathbb{C}_{\infty}}$. Sei $\partial: \operatorname{End}_{\mathbb{F}_{q}}\left(\mathbb{G}_{a / \mathbb{C}_{\infty}}\right) \longrightarrow \mathbb{C}_{\infty}$ die kanonische Abbildung. Wir fordern zusätzlich, dass das Diagramm

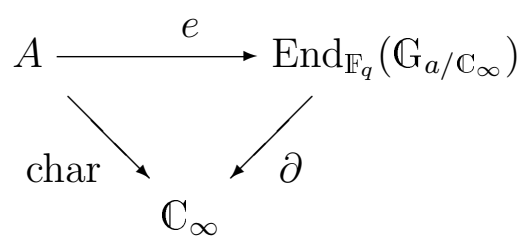

kommutiert. Die Abbildung char ist in diesem Fall die kanonische Inklusion. Anders gesagt, ein Drinfeld-Modul ist eine neue $A$-Modul-Struktur auf der additiven Gruppe $\left(\mathbb{C}_{\infty},+\right)$.

Sei $\mathbb{C}_{\infty}\{\tau\}$ der Schiefpolynomring über $\mathbb{C}_{\infty}$ mit der Relation $\tau \lambda=\lambda^{q} \tau$, dann gibt es einen kanonischen Isomorphismus

$$
\operatorname{End}_{\mathbb{F}_{q}}\left(\mathbb{G}_{a / \mathbb{C}_{\infty}}\right) \cong \mathbb{C}_{\infty}\{\tau\} .
$$

Ein Drinfeld-Modul ist also ein Ringhomomorphismus $e: A \longrightarrow \mathbb{C}_{\infty}\{\tau\}$, so dass das Diagramm

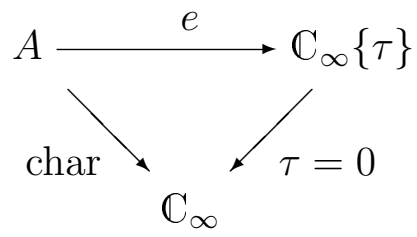

kommutiert.

Die Abbildung $e$ erzwingt für alle $0 \neq a \in A$ eine Bedingung an die Leitkoeffizienten von $e(a)$ : Es gibt eine Zahl $d \in \mathbb{N}$ mit

$$
\operatorname{deg}_{\tau} e(a)=-d \operatorname{deg}(\infty) \infty(a) .
$$

Die Zahl $d$ ist der Rang des Drinfeld-Moduls.

Ist $E$ ein Drinfeld-Modul über $\mathbb{C}_{\infty}$ von Rang $d$ und ist $0 \neq I \subsetneq A$ ein Ideal, dann erhalten wir durch

$$
E[I]:=\left\{x \in \mathbb{C}_{\infty} \mid e_{a}(x)=0 \text { für alle } x \in I\right\}
$$

die Untergruppe der I-Teilungspunkten von $\left(\mathbb{C}_{\infty},+\right)$. Es gilt

$$
\left(I^{-1} / A\right)^{d} \simeq E[I] .
$$

Die Auswahl eines Isomorphismus bezeichen wir als Level-I-Struktur.

Die Definition des Drinfeld-Moduls, der Teilungspunkte und der Level-Strukturen lässt sich, analog zum Fall der elliptischen Kurven, auf ein beliebiges Basisschema $S$ (über $\mathbb{F}_{q}$ ) verallgemeinern. 


\section{Elliptische Garben}

In die Definition eines Drinfeld-Moduls über einem Ring $R$ geht die Kurve $X$ nur durch den Funktionenring $A$ ein. Die Stelle $\infty$ liefert dennoch durch den Grad des Elements $e(a)$ Informationen. Dies führt zur Konstruktion eines auf der parametrisierten Kurve $X \times \operatorname{spec} R$ definierten Objekts, einer elliptischen Garbe. Entscheidend ist hierfür die Beobachtung, dass der Schiefpolynomring $R\{\tau\}$ durch die Drinfeld-Modul-Struktur ein projektiver $R \otimes_{\mathbb{F}_{q}} A$-Modul ist. Drinfeld führt die Konstruktion von elliptischen Garben zu Drinfeld-Moduln in seinem Artikel Commutative subrings of certain noncommutative rings ([Dri86]) durch und zeigt die Äquivalenz der zugehörigen Kategorien. Im Artikel Varieties of Modules of F-Sheaves ([Dri87]) beschreibt er zusätzlich die Konstruktion von Level-Strukturen.

Der Inhalt dieser Arbeit ist eine ausführliche Beschreibung der von Drinfeld in loc. cit. beschriebenen Konstruktionen. Außerdem wird die Konstruktion auf ein beliebiges Basisschema $S$ verallgemeinert. Außerhalb der Charakteristik wird eine Definition von Level-Strukturen für elliptische Garben, die zu einem Drinfeld-Modul gehören, beschrieben. Im Fall, dass die Charakteristik des Drinfeld-Moduls die Punkte des Levels trifft, wird darüber hinaus eine Definition vorgeschlagen.

\section{Gliederung}

In Kapitel 2 werden die grundlegenden Begriffe und Methoden der kommutativen Algebra und der algebraischen Geometrie erläutert, soweit sie in der vorliegenden Arbeit benötigt werden. Aus Gründen der Übersichtlichkeit werden jedoch die meisten Beweise nur zitiert oder es handelt sich gar um Übungsaufgaben ${ }^{1}$ in den angegebenen Büchern.

Von entscheidender Bedeutung sind im Verlauf der Arbeit die Sätze zum Basiswechsel der Kohomologie von parametrisierten Kurven und die Vertauschung des induktiven Limes mit dem globalen Schnittfunktor. Außerdem beschreibt der Autor eine im späteren Verlauf verwendete Konstruktion von Vektorbündeln auf Kurven.

Das Kapitel 3 beschäftigt sich mit der Definition von Drinfeld-Moduln über einem beliebigen Basisschema. Der Autor verwendet hier die präzisen Definitionen und Begriffe der Habilitationsschrift von T. Lehmkuhl ([Leh00]). Mit Hilfe der in der Arbeit von A. Blum und U. Stuhler Drinfeld Modules and Elliptic Sheaves beschriebenen Anleitung werden dann zu einem DrinfeldModul Vektorbündel auf der parametrisierten Kurve konstruiert. Diese werden sich im weiteren Verlauf als elliptische Garben erweisen. Alternativ wird

\footnotetext{
${ }^{1}$ Der Autor ist davon überzeugt, sie gelöst zu haben.
} 
auch eine Proj-Konstruktion der Vektorbündel beschrieben. Der Autor zeigt, dass sie mit der vorherigen Konstruktion übereinstimmt.

Die Ausarbeitung des Artikels von V.G. Drinfeld ([Dri86]) wird in Kapitel 4 durchgeführt. Es werden die auch für sich selbst interessanten Vektorbündel von allgemeinem Typ definiert und einige einfache Eigenschaften vorgestellt. Kapitel 5 definiert die elliptischen Garben. Die Kategorienäquivalenz zwischen Drinfeld-Moduln (in Standardform) und den elliptischen Garben für die eine zusätzliche Bedingung erfüllt ist, wird hier gezeigt.

Die Definition von Level-Strukturen für Drinfeld-Moduln und für elliptische Garben ist der Inhalt von Kapitel 6. Zunächst wird auf ein Problem in der Definition der Level-Struktur eines Drinfeld-Moduls im Fall, dass die Charakteristik des Drinfeld-Moduls die Punkte des Levels trifft, hingewiesen und dieses für eine reduzierte Basis auch gelöst. Im weiteren Verlauf des Kapitels wird dann die kategorielle Äquivalenz zwischen Drinfeld-Moduln mit LevelStruktur und elliptischen Garben mit Level-Struktur bewiesen. Dies bildet das Hauptresultat der vorliegenden Arbeit.

Im abschließenden Kapitel 7 wird mit den $\mathscr{D}$-elliptischen Garben, die erstmalig im Artikel D-elliptic sheaves and the Langlands correspondence [LRS93] definiert worden sind, eine Verallgemeinerung der elliptischen Garben vorgestellt. Sie waren die eigentliche Motivation für die vorliegende Arbeit. Ein Anliegen des Autors war es, eine Definition von Level-Strukturen $\mathscr{D}$-elliptischer Garben zu finden, die auch im Fall, dass die Charakteristik der $\mathscr{D}$-elliptischen Garbe die Punkte des Levels trifft, gilt. Zum Schluss des Kapitels wird eine Idee dieses Problem zu lösen vorgestellt.

\section{Danksagung}

Ich möchte an dieser Stelle Prof. Ulrich Stuhler danken, der mir nicht nur großartige Mathematik nahegebracht hat, sondern mir auch den nötigen Rückhalt gab, die vorliegende Arbeit zu vollenden. Für viele wichtige Anregungen und Kommentare bin ich Dr. Thomas Lehmkuhl sehr dankbar. Bedanken möchte ich mich auch bei Andrea Pfitzner für die sorgfältige Lektüre und orthographische Korrektur meines Textes. 


\section{Algebraische Kurven und Vektorbündel}

\subsection{Algebraische Hilfsmittel}

Alle auftretenden Ringe sind kommutativ mit Einselement.

\section{Definition 2.1}

Sei $A$ ein Ring und sei $R$ eine A-Algebra. Ein Element $x \in R$ heißt ganz über $A$, falls eine der folgenden äquivalenten Bedingungen erfüllt ist:

- $x$ ist Nullstelle eines normierten Polynoms aus $A[X]$.

- Die Unteralgebra $A[x]$ von $R$ ist ein endlich erzeugter $A$-Modul.

- Es gibt einen treuen A[x]-Modul, der als A-Modul endlich erzeugt ist.

\section{Satz 2.2}

1) Sei $B$ ganz über $A$, dann ist $B \otimes_{A} R$ ganz über $R$ (siehe [Bou98], Kapitel $V, \S 1.1$, Proposition 5, Seite 30\%).

2) Sei $A$ ein Ring, $S \subset A$ multiplikativ abgeschlossen, und sei $R$ eine $A$ Algebra. Sei $A^{\prime}$ der ganze Abschluss von $A$ in $R$. Dann ist $S^{-1} A^{\prime}$ der ganze Abschluss von $S^{-1} A$ in $S^{-1} R$ ([Bou98], Kapitel V, $\$ 1.5$., Proposition 16, Seite 314).

3) Sei $A$ ein ganz abgeschlossener Integritätsring, $K=\operatorname{Quot}(A)$ und $L / K$ eine endliche, separable $K$-Algebra. Ist $B$ der ganze Abschluss von $A$ in $L$, dann ist $B$ in einem endlich erzeugten A-Modul enthalten ([Bou98] Kapitel V, \$1.6., Propositon 18, Seite 317).

4) Sei K ein Körper, und sei $R$ eine ganz abgeschlossene K-Algebra. Sei L/K eine separable Erweiterung von $K$. Ist $L \otimes_{K} R$ ein Integritätsring, dann ist $L \otimes_{K} R$ ganz abgeschlossen ([Bou98] Kapitel V, § 1.7., Proposition 19, Seite 318).

5) Sei L/K eine algebraische Körpererweiterung. Sei v eine Bewertung von $K$ mit Bewertungsring A. Sei $B$ der ganze Abschluss von $A$ in L. Sei

$$
\begin{aligned}
\mathcal{B} & :=\left\{\text { Bewertungsringe } B_{w} \text { von } L \text { die über v liegen }\right\} \\
\mathcal{M}: & :=\{\text { Maximale Ideale } \mathfrak{m} \text { von } B\} .
\end{aligned}
$$

Es gibt eine Bijektion:

$$
\begin{aligned}
\mathcal{B} & \longrightarrow \mathcal{M} \\
B_{w} & \longmapsto \mathfrak{m}\left(B_{w}\right) \cap B
\end{aligned}
$$$$
\mathcal{M} \longrightarrow \mathcal{B}
$$$$
\mathfrak{m} \longmapsto B_{\mathfrak{m}}
$$ 
([Bou98] Kapitel VI, \$8.6., Proposition 6, Seite 427).

\section{Definition 2.3}

Ein Ring A heißt Dedekindring, falls eine der folgenden äquivalenten Bedingungen erfüllt ist:

- A ist ein noetherscher Integritätsring und alle Lokalisierungen $A_{\mathfrak{p}}$ sind Hauptidealringe für alle Primideale $\mathfrak{p} \in \operatorname{spec} A$.

- A ist normaler, noetherscher Integritätsring der Dimension 0 oder 1.

Vgl. [Liu02], Kapitel 1, Seite 11 und Kapitel 4, Definition 1.2, Seite 115.

\section{Bemerkung 2.4}

Ist $A$ ein Dedekindring, dann ist jede Lokalisierung von $A$ ein Dedekindring.

\section{Satz 2.5}

Sei $A$ ein Dedekindring und sei $M$ ein $A$-Modul. Der Modul $M$ ist genau dann flach, wenn er torsionsfrei ist.

Beweis Siehe [Liu02], Kapitel 1, Korollar 2.14, Seite 11.

\section{Lemma 2.6}

Sei $K$ ein Körper und sei $A$ eine $K$-Algebra. Sei $R$ eine weitere $K$-Algebra und sei $R \longrightarrow A \otimes_{K} R$ die kanonische Einbettung. Dann gilt:

1) $A \otimes_{K} R$ ist eine flache $R$-Algebra.

2) Ist $M$ ein flacher $A \otimes_{K} R$-Modul, dann ist $M$ ein flacher $R$-Modul.

Beweis Siehe [Mat86], §3, Seite 46.

\section{Satz 2.7}

Sei $R$ ein reduzierter, noetherscher Ring und sei $M$ ein endlich erzeugter $R$-Modul. Ist $\operatorname{dim}_{k(\mathfrak{p})} M(\mathfrak{p})=n$ konstant für alle $\mathfrak{p} \in \operatorname{spec} R$, dann ist $M$ ein projektiver $R$-Modul von Rang $n$. Hierbei sei $k(\mathfrak{p}):=R_{\mathfrak{p}} / \mathfrak{p} R_{\mathfrak{p}}$ und $M(\mathfrak{p}):=$ $M_{\mathfrak{p}} / \mathfrak{p} M_{\mathfrak{p}}$.

Beweis Man zeigt, dass $M_{\mathfrak{p}}$ ein freier $R_{\mathfrak{p}^{-}}$-Modul für alle $\mathfrak{p} \in \operatorname{spec} R$ ist. Sei also $\mathfrak{p} \in \operatorname{spec} R$. Nach [Mat86], §2, Theorem 2.3 gibt es ein minimales Erzeugendensystem $m_{1}, \ldots, m_{n}$ von $M_{\mathfrak{p}}$. Angenommen, es gäbe eine nicht triviale Relation $\sum_{i=1}^{n} r_{i} m_{i}=0$, wobei ohne Einschränkung $r_{1} \neq 0$ gewählt sei. Da mit $R$ auch $R_{\mathfrak{p}}$ reduziert ist, gibt es ein Primideal $\mathfrak{q} \subseteq \mathfrak{p}$ mit $r_{1} \notin \mathfrak{q}$, und $r_{1}$ ist eine Einheit in $R_{\mathfrak{q}}$. Mit Hilfe der Relation ist also $m_{2}, \ldots, m_{n}$ ein Erzeugendensystem von $M_{\mathfrak{q}}$ im Widerspruch zur Minimalität des Erzeugendensystems. 


\section{Bemerkung 2.8}

In Satz 2.7 reicht es aus, die Konstanz der Faserdimension für alle maximalen Ideale $\mathfrak{m} \in \operatorname{spec} R$ zu überprüfen.

Beweis Sei $\mathfrak{p} \in \operatorname{spec} R$ ein Primideal. Dann gibt es ein maximales Ideal $\mathfrak{m} \in \operatorname{spec} R$ mit $\mathfrak{p} \subseteq \mathfrak{m}$. Aus

$$
R_{\mathfrak{p}} \cong\left(R_{\mathfrak{m}}\right)_{\mathfrak{p} R_{\mathfrak{m}}}
$$

folgt die Behauptung.

\section{Theorem 2.9}

Sei $R$ Ring, $I$ ein nilpotentes Ideal in $R$ und $M$ ein $R$-Modul. Dann sind äquivalent:

1) $M$ ist flacher R-Modul.

2) $M / I M$ ist flacher $R / I$-Modul und $I \otimes_{R} M=I M$.

Beweis Siehe [Mat86], §22, Theorem 22.3, Seite 174.

\section{Theorem 2.10}

Sei $M$ ein $R$-Modul. Ist $M$ projektiv, dann ist $M_{\mathfrak{p}}$ ein freier $R_{\mathfrak{p}}$-Modul für alle $\mathfrak{p} \in \operatorname{spec} R$.

Beweis Siehe [Eis94], Aufgabe 4.11 a), Seite 136 und [Kap58], Abschnitt 4, Theorem 2, Seite 374.

\section{Satz 2.11}

Sei $M$ ein $R$-Modul von endlicher Darstellung. Dann ist $M$ genau dann projektiv, wenn $M$ ein flacher $R$-Modul ist. Ein projektiver Modul ist immer flach.

Beweis Siehe [Eis94], Kapitel 6, Aufgabe 6.2, Seite 172.

\section{Bemerkung 2.12}

Ein flacher Modul ist im Allgemeinen nicht projektiv. Beispielsweise ist $\mathbb{Q}$ kein projektiver $\mathbb{Z}$-Modul.

\section{Lemma 2.13}

Sei

$$
0 \longrightarrow L \longrightarrow M \longrightarrow N \longrightarrow 0
$$

eine exakte Sequenz von $R$-Moduln. Sind $M$ und $N$ flache $R$-Moduln, dann ist auch L ein flacher R-Modul (vgl. [Wei94], Kapitel 3, Aufgabe 3.2.2, Seite 69). 
Beweis Sei $P$ ein beliebiger $R$-Modul. Wir tensorieren die Sequenz mit $\otimes_{R} P$ und erhalten die exakte Sequenz

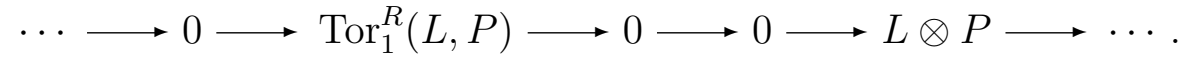

Es folgt $\operatorname{Tor}_{1}^{R}(L, P)=0$ und damit die Behauptung.

\subsection{Geometrische Hilfsmittel}

\section{Definition 2.14}

Sei K ein Körper. Eine affine Varietät ist ein affines Schema, das zu einer endlich erzeugten K-Algebra korrespondiert. Eine algebraische Varietät über einem Körper $K$ ist ein Schema $X$ über $K$, so dass es eine endliche, offene Überdeckung durch affine Varietäten gibt (vgl. [Liu02], Kapitel 2, Definition 3.47, Seite 55).

\section{Definition 2.15}

Sei $X$ ein lokal noethersches Schema. Ein Punkt $x \in X$ heißt regulär, falls $\mathcal{O}_{X, x}$ ein regulärer lokaler Ring ist. $X$ heißt regulär, falls jeder Punkt $x \in X$ regulär ist (vgl. [Liu02], Kapitel 4, Definition 2.8, Seite 128).

\section{Definition 2.16}

Sei $X$ eine algebraische Varietät über K. Sei $K^{\text {alg }}$ ein algebraischer Abschluss von $K$. Ein Punkt $x \in X$ heißt glatt, falls alle Urbilder von $x$ in $X_{K^{\text {alg }}}$ reguläre Punkte sind. $X$ heißt glatt, falls $X_{K^{\text {alg }}}$ regulär ist (vgl. [Liu02], Kapitel 4, Definition 3.28, Seite 141).

\section{Satz 2.17}

1) Sei $X$ eine algebraische Varietät über $K$. Ist $x \in X$ glatt, dann ist $x$ auch regulär (vgl. [Liu02], Kapitel 4, Korollar 3.32, Seite 142).

2) Sei X eine algebraische Varietät über einem perfekten Körper K. Dann ist $X$ genau dann glatt, wenn $X$ regulär ist (vgl. [Liu02], Kapitel 4, Korollar 3.33, Seite 142).

\section{Definition 2.18}

Sei $Y$ ein lokal noethersches Schema, und sei $f: X \longrightarrow Y$ ein Morphismus von endlichem Typ. Die Abbildung $f$ heißt glatt im Punkt $x \in X$, falls die Faser $X_{y} \longrightarrow \operatorname{spec} k(y)$ glatt ist. Hierbei sei $y:=f(x)$ (vgl. [Liu02], Kapitel 4, Definition 3.35, Seite 142).

\section{Satz 2.19}

Glatte Morphismen sind stabil unter Basiswechsel, Komposition und Faserprodukten (vgl. [Liu02] Kapitel 4, Proposition 3.38, Seite 143). 


\section{Definition 2.20}

Sei $X$ ein Schema. Ein Punkt $x \in X$ heißt normal, falls $\mathcal{O}_{X, x}$ ein ganz abgeschlossener Integritätsring ist. $X$ heißt normal, falls $X$ irreduzibel und normal für alle $x \in X$ ist (vgl. [Liu02] Kapitel 4, Definition 1.1, Seite 115).

\section{Satz 2.21}

1) Sei $X$ eine algebraische Varietät über einem Körper $K$ und sei $L$ eine beliebige Körpererweiterung von K. Sei $X$ geometrisch reduziert (oder geometrisch ganz, - irreduzibel, - zusammenhängend). Dann ist auch $X_{L}$ geometrisch reduziert (oder geometrisch ganz, - irreduzibel, - zusammenhängend).

2) Sei $X$ eine geometrisch ganze, algebraische Varietät über einem Körper $K$. Sei $Y$ eine ganze Varietät über K. Dann ist auch $X \times_{K} Y$ ganz. Selbiges gilt für reduziert, irreduzibel, zusammenhängend.

Beweis Siehe [Liu02], Kapitel 3, Aufgabe 2.14, 2.15, Seite 97, 98.

\section{Lemma 2.22}

Sei $: X \longrightarrow Y$ ein Morphismus von Schemata. Sei $\mathscr{F}$ eine quasi-kohärente $\mathcal{O}_{X}$-Modulgarbe und sei $\mathscr{G}$ eine quasi-kohärente $\mathcal{O}_{Y}$-Modulgarbe. Es gilt

1) Ist ८ eine Einbettung, dann gibt einen kanonischen Isomorphismus

$$
\iota^{*} \iota_{*} \mathscr{G} \cong \mathscr{G} .
$$

2) Ist $\iota: Y \longrightarrow X$ eine affine Abbildung, dann gibt es einen kanonischen Isomorphismus

$$
\mathscr{F} \otimes_{\mathcal{O}_{X}} \iota_{*} \mathcal{O}_{Y} \cong \iota_{*} \iota^{*} \mathscr{F}
$$

3) Sei $\mathscr{I} \subseteq \mathcal{O}_{X}$ eine quasi-kohärente Idealgarbe mit $\mathscr{I} \mathscr{F}=0$ und sei $\iota:$ $Y \longrightarrow X$ die zugehörige abgeschlossene Einbettung. Dann gibt es einen kanonischen Isomorphismus $\mathscr{F} \cong \iota_{*} \iota^{*} \mathscr{F}$.

Beweis Die Behauptungen 1) und 2) ergeben sich aus [Liu02], Kapitel 5, Aufgabe 1.1, Seite $171 \mathrm{f}$.

Da eine abgeschlossene Einbettung auch affin ist, können wir zum Beweis von 3) den Teil 2) anwenden. Es folgt

$$
\begin{aligned}
\iota_{*} \iota^{*} \mathscr{F} & \cong \mathscr{F} \otimes_{\mathcal{O}_{X}} \iota_{*} \mathcal{O}_{Y} \\
& \cong \mathscr{F} \otimes_{\mathcal{O}_{X}} \mathcal{O}_{X} / \mathscr{I} \\
& \cong \mathscr{F} / \mathscr{I} \mathscr{F} \\
& \cong \mathscr{F} .
\end{aligned}
$$




\section{Lemma 2.23}

Sei $f: X \longrightarrow S$ ein Morphismus von Schemata und sei $\mathscr{E}$ ein quasikohärenter $\mathcal{O}_{X}$-Modul. Sei $g: T \longrightarrow S$ ein Basiswechsel. Wir erhalten das Diagramm

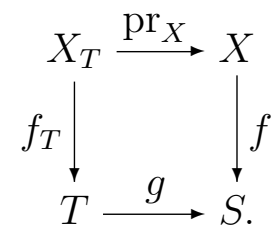

1) Ist $f$ separiert und quasi-kompakt und ist $g$ ein flacher Basiswechsel, dann gibt es einen kanonischen Isomorphismus

$$
g^{*} f_{*} \mathscr{E} \cong f_{T *} \operatorname{pr}_{X}^{*} \mathscr{E}
$$

2) In der Situation von Teil 1) sei $\mathscr{F}$ ein lokal freier $\mathcal{O}_{S}$-Modul. Dann gilt die Projektionsformel

$$
f_{*} \mathscr{E} \otimes_{\mathcal{O}_{S}} \mathscr{F} \cong f_{*}\left(\mathscr{E} \otimes_{\mathcal{O}_{X}} f^{*} \mathscr{F}\right)
$$

Beweis Siehe [Liu02], Kapitel 5, Aufgabe 1.16 b), c) Seite 174 und 175.

\subsection{Glatte und irreduzible algebraische Kurven}

\section{Definition 2.24}

Sei K ein Körper. Eine algebraische Varietät X über $K$ heißt Kurve, falls jede irreduzible Komponente 1-dimensional ist (vgl. [Liu02], Kapitel 2, Definition 5.29, Seite 75).

\section{Satz 2.25}

Sei $X$ irreduzible, glatte Kurve über einem Körper K. Dann sind für alle affinen, offenen Teilmengen $U \subseteq X$ und für alle $x \in X$ die Ringe $\mathcal{O}_{X}(U)$ und $\mathcal{O}_{X, x}$ Dedekindringe.

Beweis Ist $R$ ein regulärer, 0- oder 1-dimensionaler lokaler Ring, dann ist $R$ ein Hauptidealring. Die Behauptung ergibt sich direkt aus den Definitionen.

\section{Folgerung 2.26}

Sei X eine geometrisch irreduzible, glatte Kurve über einem Körper K. Sei $L$ eine Körpererweiterung von $K$. Dann ist auch $X \times_{K} L$ eine geometrisch irreduzible, glatte Kurve über L. 


\subsection{Projektive algebraische Kurven}

Sei im Folgenden $X$ eine glatte, projektive, absolut irreduzible Kurve über einem Körper $K$. Sei $\infty \in X$ ein abgeschlossener Punkt. Sei $\mathcal{O}_{X}(\infty)$ das zum Punkt (Divisor) $\infty$ gehörende Geradenbündel.

\section{Satz 2.27}

Sei $P \in X$ ein abgeschlossener Punkt. Dann ist $X \backslash\{P\}$ eine affine, offene Teilmenge von $X$.

Beweis Siehe [Har], The Theorem of Riemann-Roch, Seite 248.

\section{Satz 2.28}

Es ist $H^{0}\left(X, \mathcal{O}_{X}\right)=K$.

Beweis Siehe [Liu02], Kapitel 3, Folgerung 3.21, Seite 105.

\section{Satz 2.29}

Das Geradenbündel $\mathcal{O}_{X}(\infty)$ ist ampel.

Beweis Es ist $\operatorname{deg} \mathcal{O}_{X}(\infty)=\operatorname{deg}(\infty)>0$. Die Behauptung folgt nun aus [Liu02], Kapitel 7, Satz 5.5, Seite 305.

\section{Satz 2.30}

Es gibt eine Zahl $m \in \mathbb{N}$, so dass $\mathcal{O}_{X}(\infty)^{\otimes m} \cong \mathcal{O}_{X}(m \infty)$ sehr ampel ist. Das heißt, es gibt eine abgeschlossene Einbettung $f: X \longrightarrow \mathbb{P}_{K}^{n}$, so dass gilt

$$
\mathcal{O}_{X}(m \infty) \cong f^{*} \mathcal{O}_{\mathbb{P}_{K}^{n}}(1) \text {. }
$$

Beweis Siehe [Liu02], Kapitel 5, Theorem 1.34, Seite 169.

Satz 2.31

Sei $\mathscr{E}$ ein Vektorbündel ${ }^{2}$ auf $X$ und sei $U:=X \backslash\{\infty\}$. Für alle $n \in \mathbb{Z}$ seien

$$
H^{0}(X, \mathscr{E}(n \infty)) \longrightarrow H^{0}(U, \mathscr{E}(n \infty))=H^{0}(U, \mathscr{E})
$$

die kanonischen Abbildungen, und für alle $n, m \in \mathbb{Z}, n \leq m$, seinen

$$
H^{0}(X, \mathscr{E}(n \infty)) \longrightarrow H^{0}(X, \mathscr{E}(m \infty))
$$

die kanonischen Inklusionen. Dann gilt

$$
\underset{n}{\lim _{n}} H^{0}(X, \mathscr{E}(n \infty)) \cong H^{0}(U, \mathscr{E}) .
$$

\footnotetext{
${ }^{2}$ Die Aussage gilt auch für einen quasi-kohärenten $\mathcal{O}_{X}$-Modul.
} 
Beweis Sei $e:=1 \in H^{0}\left(X, \mathcal{O}_{X}(\infty)\right)$. Dann gilt in der Notation von [Liu02], Kapitel 5, Definition 1.24, Seite 166

$$
X_{e}:=\left\{x \in X \mid \mathcal{O}_{X}(\infty)_{x}=e_{x} \mathcal{O}_{X, x}\right\}=U
$$

Die Behauptung ergibt sich nun direkt aus [Liu02], Kapitel 5, Lemma 1.25., a) und b), Seite 166 .

\section{Lemma 2.32}

Sei $B=\bigoplus_{i=0}^{\infty} B_{i}$ eine graduierte Algebra. Dann gibt es für alle $n \in \mathbb{N}$ einen kanonischen Isomorphismus

$$
\operatorname{Proj} B \cong \operatorname{Proj}\left(\bigoplus_{i=0}^{\infty} B_{i n}\right) .
$$

Ist $B$ eine endlich erzeugte $B_{0}$-Algebra, dann gibt es eine Zahl $n_{0}$, so dass die graduierte Algebra $\bigoplus_{i=0}^{\infty} B_{\text {in }}$ für jedes $n \geq n_{0}$ von $B_{n}$ erzeugt wird.

Beweis Siehe [Liu02], Kapitel 2, Aufgabe 3.11, Seite 57 und [Bou98], Kapitel III, § 1.3, Proposition 3 .

Definition 2.33 ([GD61], Abschnitt 3.3)

Sei $B$ ein graduierter Ring und sei $X:=\operatorname{Proj} B$. Sei $\mathscr{E}$ ein $\mathcal{O}_{X}$-Modul. Wir definieren durch

$$
\Gamma_{*}(\mathscr{E}):=\bigoplus_{n \in \mathbb{Z}} H^{0}(X, \mathscr{E}(n))
$$

den $z u \mathscr{E}$ assoziierten, graduierten $B$-Modul.

\section{Satz 2.34}

Sei $B$ ein graduierter Ring, der als $B_{0}$-Algebra endlich erzeugt ist. Sei $X:=$ Proj $B$ und sei $\mathscr{E}$ eine quasi-kohärente $\mathcal{O}_{X}$-Modulgarbe. Dann gibt es einen kanonischen Isomorphismus

$$
\Gamma_{*}(\mathscr{E})^{\sim} \cong \mathscr{E}
$$

Beweis Nach Lemma 2.32 können wir ohne Einschränkung der Allgemeinheit annehmen, dass $B$ von $B_{1}$ als $B_{0}$-Algebra erzeugt wird. Die Aussage folgt nun aus [Har77], II, Kapitel 5, Proposition 5.15, Seite 119.

\section{Definition 2.35}

Sei

$$
\mathcal{S}_{X}:=\bigoplus_{i=0}^{\infty} H^{0}\left(X, \mathcal{O}_{X}(i \infty)\right)
$$




\section{Lemma 2.36}

$\mathcal{S}_{X}$ ist eine endlich erzeugte, graduierte $K$-Algebra.

Beweis Nach Satz 2.28 gilt $H^{0}\left(X, \mathcal{O}_{X}\right)=K$. Für alle $i, j \in \mathbb{Z}$ gibt es kanonische Abbildungen

$$
\mathcal{O}_{X}(i \infty) \otimes_{\mathcal{O}_{X}} \mathcal{O}_{X}(j \infty) \longrightarrow \mathcal{O}_{X}((i+j) \infty)
$$

Diese definieren die Struktur einer graduierten $K$-Algebra auf $\mathcal{S}_{X}$. Für eine Kurve $X$ über $K$ und eine affine, offene Teilmenge $U$ ist $H^{0}\left(U, \mathcal{O}_{X}\right)$ eine endlich erzeugte $K$-Algebra. Ist $f_{1}, \ldots, f_{n} \in H^{0}\left(U, \mathcal{O}_{X}\right)$ ein Erzeugendensystem, dann gibt es nach Satz 2.31 eine Zahl $n_{0} \in \mathbb{N}$ mit $f_{1}, \ldots, f_{n} \in$ $H^{0}\left(X, \mathcal{O}_{X}\left(n_{0} \infty\right)\right)$. Insbesondere erzeugen die Elemente $\mathcal{S}_{X}$ als graduierte $K$ Algebra.

\section{Satz 2.37}

Es gilt $\operatorname{Proj} \mathcal{S}_{X} \cong X$.

Beweis Nach Satz 2.30 gibt es eine Zahl $m$ und eine Einbettung $f: X \longrightarrow \mathbb{P}_{K}^{n}$ mit $f^{*}\left(\mathcal{O}_{\mathbb{P}_{K}^{n}}(1)\right) \cong \mathcal{O}_{X}(\infty)$. Die Einbettung $f$ induziert eine exakte Sequenz der Form

$$
0 \longrightarrow \mathscr{I} \longrightarrow \mathcal{O}_{\mathbb{P}_{K}^{n}} \stackrel{f^{\sharp}}{\longrightarrow} f_{*} \mathcal{O}_{X} \longrightarrow 0
$$

mit $\mathscr{I}:=\operatorname{Ker} f^{\sharp}$. Für alle $i>0$ tensorieren wir die Sequenz mit $\mathcal{O}_{\mathbb{P}_{K}^{n}}(i)$ und erhalten mit Hilfe der Projektionsformel (vgl. Lemma 2.23, 2)

$$
f_{*}\left(\mathcal{O}_{X} \otimes_{\mathcal{O}_{X}} f^{*} \mathcal{O}_{\mathbb{P}_{K}^{n}}(i)\right) \cong f_{*} \mathcal{O}_{X} \otimes_{\mathcal{O}_{\mathbb{P}_{K}^{n}}^{n}} \mathcal{O}_{\mathbb{P}_{K}^{n}}(i)
$$

die Sequenz

$$
0 \longrightarrow \mathscr{I}(i) \longrightarrow \mathcal{O}_{\mathbb{P}_{K}^{n}}(i) \longrightarrow f_{*}\left(\mathcal{O}_{X}(i)\right) \longrightarrow 0 .
$$

Der Übergang zu den globalen Schnitten liefert mit Hilfe von

$$
f_{*}\left(\mathcal{O}_{X}(i)\right)\left(\mathbb{P}_{K}^{n}\right)=H^{0}\left(X, \mathcal{O}_{X}(m i \infty)\right)
$$

die Sequenz

$$
\begin{aligned}
0 \longrightarrow H^{0}\left(\mathbb{P}_{K}^{n}, \mathscr{I}(i)\right) & \longrightarrow H^{0}\left(\mathbb{P}_{K}^{n}, \mathcal{O}_{\mathbb{P}_{K}^{n}}(i)\right) \longrightarrow H^{0}\left(X, \mathcal{O}_{X}(\operatorname{mi\infty })\right) \longrightarrow \\
& \longrightarrow H^{1}\left(\mathbb{P}_{K}^{n}, \mathscr{I}(i)\right) \longrightarrow \cdots .
\end{aligned}
$$

Es gibt eine Zahl $d>0$ mit $H^{1}\left(\mathbb{P}_{K}^{n}, \mathscr{I}(i)\right)=0$ für alle $i \geq d$. Für $i \geq d$ gilt also

$$
H^{0}\left(\mathbb{P}_{K}^{n}, \mathcal{O}_{\mathbb{P}_{K}^{n}}(i)\right) / H^{0}\left(\mathbb{P}_{K}^{n}, \mathscr{I}(i)\right) \cong H^{0}\left(X, \mathcal{O}_{X}(m i \infty)\right)
$$


Des Weiteren ist $H^{0}\left(\mathbb{P}_{K}^{n}, \mathscr{I}\right)=0$, da anderenfalls $\mathscr{I}=\mathcal{O}_{\mathbb{P}_{K}^{n}}$ gilt. Nach Satz 2.28 gilt $H^{0}\left(X, \mathcal{O}_{X}\right)=K$. Es folgt

$$
\begin{aligned}
\operatorname{Proj}\left(\bigoplus_{i=0}^{\infty} H^{0}\left(X, \mathcal{O}_{X}(i \infty)\right)\right) & \cong \operatorname{Proj}\left(\bigoplus_{i=0}^{\infty} H^{0}\left(X, \mathcal{O}_{X}(\text { mdi })\right)\right) \\
& \cong \operatorname{Proj}\left(\bigoplus_{i=0}^{\infty} H^{0}\left(\mathbb{P}_{K}^{n}, \mathcal{O}_{\mathbb{P}_{K}^{n}}(d i)\right) / H^{0}\left(\mathbb{P}_{K}^{n}, \mathscr{I}(d i)\right)\right) \\
& \cong \operatorname{Proj}\left(\bigoplus_{i=0}^{\infty} H^{0}\left(\mathbb{P}_{K}^{n}, \mathcal{O}_{\mathbb{P}_{K}^{n}}(i)\right) / H^{0}\left(\mathbb{P}_{K}^{n}, \mathscr{I}(i)\right)\right)
\end{aligned}
$$

Nach [Har77], Teil II, Proposition 5.13, 5.15, Folgerung 5.16, Seite 118 ff gilt

$$
\begin{aligned}
\operatorname{Proj}\left(\bigoplus_{i=0}^{\infty} H^{0}\left(\mathbb{P}_{K}^{n}, \mathcal{O}_{\mathbb{P}_{K}^{n}}(i)\right) / H^{0}\left(\mathbb{P}_{K}^{n}, \mathscr{I}(i)\right)\right) & \cong \operatorname{Proj}\left(K\left[x_{0}, \ldots, x_{n}\right] / \Gamma_{*}(\mathscr{I})\right) \\
& \cong X
\end{aligned}
$$

Es folgt die Behauptung.

\section{Folgerung 2.38}

Es gilt für alle $j \in \mathbb{Z}$

$$
\left(\mathcal{S}_{X}[j]\right)^{\sim} \cong \mathcal{O}_{X}(j \infty)
$$

Beweis Nach Satz 2.34 gilt

$$
\Gamma_{*}\left(\mathcal{O}_{X}(j \infty)\right)^{\sim} \cong \mathcal{O}_{X}(j \infty)
$$

Es ist $\mathcal{O}_{X}(1)=\mathcal{O}_{X}(m \infty)$ und es folgt

$$
\bigoplus_{i=0}^{\infty} H^{0}\left(\mathcal{O}_{X}(j \infty) \otimes \mathcal{O}_{X}(i m \infty)\right) \cong \bigoplus_{i=0}^{\infty} H^{0}\left(\mathcal{O}_{X}(j+i m \infty)\right)
$$

Nach [Har77], Teil II, Aufgabe 5.9, Seite 125 gilt

$$
\left(\bigoplus_{i=0}^{\infty} H^{0}\left(X, \mathcal{O}_{X}(i \infty)\right)[j]\right)^{\sim} \cong\left(\bigoplus_{i=0}^{\infty} H^{0}\left(\mathcal{O}_{X}(j+i m \infty)\right)\right)^{\sim}
$$

Folgerung 2.39

Sei $A:=H^{0}\left(X \backslash \infty, \mathcal{O}_{X}\right)$ und $e:=1 \in H^{0}\left(X, \mathcal{O}_{X}(\infty)\right)$. Dann gilt

$$
\mathcal{S}_{X}\left[e^{-1}\right]_{(0)} \cong A
$$


Beweis In der Notation von [Har77], Teil II, Lemma 5.14, Seite 118, gilt $X_{e}=X \backslash \infty$. Mit loc. cit. a), b) folgt, dass die kanonische Abbildung

$$
\mathcal{S}_{X}\left[e^{-1}\right]_{(0)} \ni \frac{f}{e^{i}} \longmapsto f \in A
$$

ein Isomorphismus ist.

Folgerung 2.40

Sei e $:=1 \in H^{0}\left(X, \mathcal{O}_{X}(\infty)\right)$. Dann gilt

$$
\operatorname{Proj}\left(\mathcal{S}_{X} / e \mathcal{S}_{X}\right) \cong \operatorname{spec} K(\infty) .
$$

Beweis Es ist

$$
\left(\mathcal{S}_{X}\right) / e\left(\mathcal{S}_{X}\right) \cong \bigoplus_{i=0}^{\infty} H^{0}\left(X, \mathcal{O}_{X}(i \infty)\right) / H^{0}\left(X, \mathcal{O}_{X}((i-1) \infty)\right)
$$

Sei $\pi_{\infty}$ ein uniformisierendes Element von $\mathcal{O}_{X, \infty}$ und sei $f \in H^{0}\left(X, \mathcal{O}_{X}(i \infty)\right)$ mit $\infty(f)=-i$. Wir definieren für $i \geq 0$ die Abbildungen

$$
\begin{aligned}
H^{0}\left(X, \mathcal{O}_{X}(i \infty)\right) / H^{0}\left(X, \mathcal{O}_{X}((i-1) \infty)\right) & \longrightarrow K(\infty)[T]_{(i)} \\
f & \longmapsto\left(f \pi_{\infty}^{i} \quad \bmod \mathfrak{m}_{\infty}\right) T^{i}
\end{aligned}
$$

Für $i \gg 0$ sind die Abbildungen Isomorphismen und es folgt die Behauptung.

\subsection{Vektorbündel}

\section{Definition 2.41}

Sei $S$ ein Schema. Ein Vektorbündel $\mathscr{E}$ auf $S$ ist eine quasi-kohärente Garbe von $\mathcal{O}_{S^{-}}$Moduln, so dass für alle offenen, affinen Teilmengen $U \subseteq S$ der $\mathcal{O}_{S}(U)$-Modul $H^{0}(U, \mathscr{E})$ projektiv ist (vgl. [Dri03]).

\section{Theorem 2.42}

Sei $\mathscr{E}$ ein quasi-kohärenter $\mathcal{O}_{S}$-Modul. Dann ist $\mathscr{E}$ ein Vektorbündel, wenn es eine offene Überdeckung $\bigcup_{i \in I} U_{i}=S$ gibt, so dass $\left.\mathscr{E}\right|_{U_{i}}$ für alle $i \in I$ ein Vektorbündel über $U_{i}$ ist.

Beweis Siehe [Dri03], [RG71], §3, 3.1 Descent de la projectivité, Seite 81 ff.

\section{Bemerkung 2.43}

Die Aussage des Theorems gilt nicht nur für eine Überdeckung in der ZariskiTopologie, sondern lässt sich nach loc. cit. auch auf eine Überdeckung in der fpqc-Topologie verallgemeinern. 


\section{Lemma 2.44}

Sei $S$ ein Schema und sei

$$
0 \longrightarrow \mathscr{E} \longrightarrow \mathscr{F} \longrightarrow \mathscr{G} \longrightarrow 0
$$

eine exakte Sequenz von quasi-kohärenten $\mathcal{O}_{S}$-Moduln. Sind $\mathscr{F}$ und $\mathscr{G}$ Vektorbündel, dann ist auch $\mathscr{E}$ ein Vektorbündel.

Beweis Die Aussage lässt sich nach Theorem 2.42 lokal in der ZariskiTopologie zeigen. Sei deshalb $S=\operatorname{spec} R$ ein affines Schema. Dann spaltet die Sequenz

$$
0 \longrightarrow \mathscr{E}(S) \longrightarrow \mathscr{F}(S) \longrightarrow \mathscr{G}(S) \longrightarrow 0
$$

und $\mathscr{E}(S)$ ist direkter Summand des projektiven $R$-Moduls $\mathscr{F}(S)$ und damit projektiv.

\subsection{Kohomologie und Basiswechsel von Vektorbündeln auf parametrisierten Kurven}

Sei $S$ ein Schema über $K$, dann ist $X \times S$ eine parametrisierte Kurve über $S$. Sei $s \in S$ und $k(s)$ der Restekörper zum Punkt $s$. Mit $X_{S}$, bzw. $X_{s}$ sei das Faserprodukt $X \times S$, bzw. $X \times \operatorname{spec} k(s)$ bezeichnet. Nach Folgerung 2.26 ist mit $X$ auch $X_{s}$ eine glatte, projektive, absolut irreduzible Kurve über $k(s)$ für alle $s \in S$.

Für einen $\mathcal{O}_{X \times S^{-}}$Modul $\mathscr{E}$ sei mit $\mathscr{E}_{s}$ der Pullback der Garbe $\mathscr{E}$ auf $X \times s$ bezeichnet.

Ziel des Abschnitts ist die Verallgemeinerung des nachfolgenden Theorems auf den Fall eines nicht noetherschen Basisschemas. Um dieses Ziel zu erreichen, müssen wir zusätzliche Voraussetzungen an das Vektorbündel $\mathscr{E}$ stellen. Diese sind günstigerweise für den Fall der später betrachteten elliptischen Garben erfüllt.

Theorem 2.45

Ist $S$ ein lokal noethersches Schema, dann folgt für $k=0,1$ aus

$$
H^{k}\left(X \times s, \mathscr{E}(i \infty)_{s}\right)=0 \forall s \in S
$$

bereits $H^{k}(X \times S, \mathscr{E}(i \infty))=0$.

Beweis Siehe [Liu02], Kapitel 5, Bemerkung 3.21(c), Seite 204). 


\section{Lemma 2.46}

Sei $S=\operatorname{spec} R$ ein affines Schema und sei $\mathscr{E}$ ein Vektorbündel von Rang d auf $X \times S$. Dann gibt es einen noetherschen Unterring $R_{0} \subseteq R$, bzw. ein affines, noethersches Schema $S_{0}=\operatorname{spec} R_{0}$ und ein Vektorbündel $\mathscr{E}_{0}$ auf $X \times S_{0}$ mit

$$
\mathscr{E}_{0} \times_{S_{0}} S \cong \mathscr{E}
$$

Beweis Wesentlich ist hier die Separiertheit und die Quasi-Kompaktheit von $X \times S$. Dann lässt sich $R_{0}$ und das Bündel $\mathscr{E}_{0}$ über die Einträge in den endlich vielen Klebedaten konstruieren.

\section{Lemma 2.47}

Sei $S=\operatorname{spec} R$ ein affines Schema. Seien $U, V \subseteq X$ offene, affine Teilmengen von $X$, die $X$ überdecken. Dann berechnet der Čech-Komplex

$$
0 \longrightarrow \mathscr{E}(U \times S) \times \mathscr{E}(V \times S) \stackrel{\psi}{\longrightarrow} \mathscr{E}((U \cap V) \times S) \longrightarrow 0
$$

die Kohomologie des Vektorbündels $\mathscr{E}$.

Beweis Siehe [Liu02], Kapitel 5, Theorem 2.19, Seite 186.

\section{Lemma 2.48}

Sei $R$ ein Ring und sei $M$ ein projektiver, endlich erzeugter $R$-Modul.

1) Sei $\mathfrak{p}$ ein Primideal von $R$ dann ist

$$
M_{\mathfrak{p}}=0 \quad \Longleftrightarrow \quad M \otimes_{R} k(\mathfrak{p})=0 .
$$

2) Sei $\mathfrak{m}$ ein maximales Ideal von $R$ mit $\mathfrak{p} \subseteq \mathfrak{m}$. Dann gilt

$$
M \otimes_{R} k(\mathfrak{m})=0 \quad \Longleftrightarrow \quad M \otimes_{R} k(\mathfrak{p})=0 .
$$

Beweis zu 1) Als projektiver, endlich erzeugter $R$-Modul ist $M$ lokal frei, das heißt, es gibt eine Zahl $n \in \mathbb{N}$ mit $M_{\mathfrak{p}} \cong R_{\mathfrak{p}}^{n}$. Es folgt

$$
M \otimes_{R} k(\mathfrak{p}) \cong M_{\mathfrak{p}} \otimes_{R_{\mathfrak{p}}} k(\mathfrak{p}) \cong R_{\mathfrak{p}}^{n} \otimes_{R_{\mathfrak{p}}} k(\mathfrak{p}) \cong k(\mathfrak{p})^{n}
$$

zu 2) Nach Teil 1) reicht es zu zeigen, dass

$$
M_{\mathfrak{m}}=0 \quad \Longleftrightarrow \quad M_{\mathfrak{p}}=0
$$

gilt. Es ist

$$
\left(M_{\mathfrak{m}}\right)_{\mathfrak{p}} \cong\left(R_{\mathfrak{m}}^{n}\right)_{\mathfrak{p}} \cong R_{\mathfrak{p}}^{n} \cong M_{\mathfrak{p}}
$$

und wir erhalten die Behauptung. 


\section{Satz 2.49}

In der Situation von Lemma 2.46 sei $H^{1}\left(X \times S_{0}, \mathscr{E}_{0}\right)$ ein projektiver, endlich erzeugter $R_{0}$-Modul. Ist $H^{1}\left(X \times s, \mathscr{E}_{s}\right)=0$ für alle $s \in S$, dann ist auch $H^{1}(X \times S, \mathscr{E})=0$.

Beweis Wir fassen mit Hilfe der kanonischen Abbildung $S \longrightarrow S_{0}$ die Punkte von $S$ als Punkte von $S_{0}$ auf. Es gilt dann

$$
\mathscr{E}_{s}=\left(\mathscr{E}_{0}\right)_{s}
$$

für alle $s \in S$. Da $S_{0}$ noethersch ist, folgt nach [Liu02], Kapitel 5, Bemerkung 3.21, (b), (c), Seite 204,

$$
H^{1}\left(X \times s,\left(\mathscr{E}_{0}\right)_{s}\right)=0 \quad \Longrightarrow \quad H^{1}\left(X \times S_{0}, \mathscr{E}_{0}\right) \otimes_{R_{0}} k(s)=0
$$

für alle $s \in S$. Da $S$ dicht in $S_{0}$ liegt, folgt $H^{1}\left(X \times S_{0}, \mathscr{E}_{0}\right)=0$ aus Lemma $2.48,1)$ und 2 ). Nach Lemma 2.47 berechnet der Čech-Komplex

$$
0 \longrightarrow \mathscr{E}_{0}\left(U \times S_{0}\right) \times \mathscr{E}_{0}\left(V \times S_{0}\right) \stackrel{\psi_{0}}{\longrightarrow} \mathscr{E}_{0}\left((U \cap V) \times S_{0}\right) \longrightarrow 0
$$

die Kohomologie von $\mathscr{E}_{0}$ und deshalb ist $\psi_{0}$ surjektiv. Tensorieren der Sequenz mit $\cdot \otimes_{R_{0}} R$ liefert den Čech-Komplex zur Berechnung der Kohomologie von $\mathscr{E}$. Die Abbildung $\psi_{0} \otimes_{R_{0}} R$ bleibt aufgrund der Rechtsexaktheit des Tensorproduktes surjektiv. Es folgt die Behauptung.

\section{Satz 2.50}

In der Situation von Lemma 2.46 sei $H^{0}\left(X \times S_{0}, \mathscr{E}_{0}\right)$ ein projektiver, endlich erzeugter $R_{0}$-Modul. Sei zusätzlich $H^{1}\left(X \times S_{0}, \mathscr{E}_{0}\right)$ ein flacher $R_{0}$-Modul. Ist $H^{0}\left(X \times s, \mathscr{E}_{s}\right)=0$ für alle $s \in S$, dann ist auch $H^{0}(X \times S, \mathscr{E})=0$.

Beweis Aus dem Čech-Komplex

$$
0 \longrightarrow \mathscr{E}_{0}\left(U \times S_{0}\right) \times \mathscr{E}_{0}\left(V \times S_{0}\right) \stackrel{\psi_{0}}{\longrightarrow} \mathscr{E}_{0}\left((U \cap V) \times S_{0}\right) \longrightarrow 0
$$

entnehmen wir die exakte Sequenz

$$
0 \longrightarrow \operatorname{Im} \psi_{0} \longrightarrow \mathscr{E}_{0}\left((U \cap V) \times S_{0}\right) \longrightarrow H^{1}\left(X \times S_{0}, \mathscr{E}_{0}\right) \longrightarrow 0 .
$$

Mit $H^{1}\left(X \times S_{0}, \mathscr{E}_{0}\right)$ und $\mathscr{E}\left((U \cap V) \times S_{0}\right)(\operatorname{vgl}$. Lemma 2.6) ist nach Lemma 2.13 auch $\operatorname{Im} \psi_{0}$ ein flacher $R_{0}$-Modul. Wir tensorieren die Sequenz

$$
0 \longrightarrow H^{0}\left(X \times S_{0}, \mathscr{E}_{0}\right) \longrightarrow \mathscr{E}_{0}\left(U \times S_{0}\right) \times \mathscr{E}_{0}\left(V \times S_{0}\right) \longrightarrow \operatorname{Im} \psi_{0} \longrightarrow 0
$$

mit $\cdot \otimes_{R_{0}} R$ und erhalten wegen der Flachheit des $R_{0}$-Moduls $\operatorname{Im} \psi_{0}$

$$
H^{0}\left(X \times S_{0}, \mathscr{E}_{0}\right) \otimes_{R_{0}} R \cong H^{0}(X \times S, \mathscr{E}) .
$$

Analog zum Beweis von Satz 2.49 folgt $H^{0}\left(X \times S_{0}, \mathscr{E}_{0}\right)=0$ aus $H^{0}(X \times$ $\left.s,\left(\mathscr{E}_{0}\right)_{s}\right)=0$ für alle $s \in S$. Es folgt die Behauptung. 


\section{Folgerung 2.51}

In der Situation von Lemma 2.46 sei $H^{1}(X \times S, \mathscr{E})$ ein flacher $R$-Modul. Sei $S^{\prime}=\operatorname{spec} R^{\prime}$ ein affines Schema über $S$. Dann gilt

$$
H^{0}(X \times S, \mathscr{E}) \otimes_{R} R^{\prime} \cong H^{0}\left(X \times S^{\prime}, \mathscr{E}_{S^{\prime}}\right)
$$

und

$$
H^{1}(X \times S, \mathscr{E}) \otimes_{R} R^{\prime} \cong H^{1}\left(X \times S^{\prime}, \mathscr{E}_{S^{\prime}}\right)
$$

Beweis Nach Lemma 2.47 berechnen die Sequenzen

$$
0 \longrightarrow H^{0}(X \times S, \mathscr{E}) \longrightarrow \mathscr{E}(U \times S) \times \mathscr{E}(V \times S) \stackrel{\psi}{\longrightarrow} \operatorname{Im} \psi \longrightarrow 0
$$

und

$$
0 \longrightarrow \operatorname{Im} \psi \longrightarrow \mathscr{E}((U \cap V) \times S) \longrightarrow H^{1}(X \times S, \mathscr{E}) \longrightarrow 0 .
$$

die Kohomologie von $\mathscr{E}$. Ist $H^{1}(X \times S, \mathscr{E})$ ein flacher $R$-Modul, dann bleiben die Sequenzen unter Tensorieren mit $\otimes_{R} R^{\prime}$ exakt und berechnen die Kohomologie von $\mathscr{E}_{S^{\prime}}$.

Wir stellen zum Schluss des Abschnitts noch eine Variante des Beweises von Satz 2.50 vor.

\section{Satz 2.52 (Dualisierungssatz)}

Sei $S=\operatorname{spec} R$ ein affines Schema. Dann gibt es eine dualisierende Garbe $\omega_{S}$ bezüglich der Abbildung $X \times S \longrightarrow S$ und es gibt eine nicht ausgeartete Paarung

$$
H^{0}(X \times S, \mathscr{E}) \times H^{1}\left(X \times S, \mathscr{E}^{\vee} \otimes_{\mathcal{O}_{X \times S}} \omega_{S}\right) \longrightarrow R .
$$

Beweis Nach [Liu02], §6, Korollar 4.29, Seite 246 besitzt $X \longrightarrow \operatorname{spec} K$ eine dualisierende Garbe $\omega_{X}$. Flacher Basiswechsel liefert die Behauptung (vgl. [Liu02], Kapitel 6, Aufgabe 4.10, Seite 250). Außerdem ist $\omega_{S}:=\operatorname{pr}_{X}^{*}\left(\omega_{X}\right)$ die dualisierende Garbe.

\section{Folgerung 2.53}

Sei $S=\operatorname{spec} R$ ein affines Schema und sei $\mathscr{E}$ ein Vektorbündel auf $X \times S$. Ist $H^{0}\left(X \times s, \mathscr{E}_{s}\right)=0$ für alle $s \in S$, dann ist auch $H^{0}(X \times S, \mathscr{E})=0$.

Beweis Mit Hilfe des Dualisierungssatzes 2.52 folgt

$$
0=H^{0}\left(X \times s, \mathscr{E}_{s}\right)=H^{1}\left(X \times s, \mathscr{E}_{s}^{\vee} \otimes_{\mathcal{O}_{X \times s}} \omega_{s}\right)
$$

für alle $s \in S$. Nach Satz 2.49 folgt $H^{1}\left(X \times S, \mathscr{E} \vee \otimes_{\mathcal{O}_{X \times S}} \omega_{S}\right)=0$. Wir wenden nun den Dualisierungssatz 2.52 ein weiteres Mal an und erhalten die Behauptung. 


\subsection{Twists von Vektorbündeln auf parametrisierten Kurven}

\section{Definition 2.54}

Sei $\mathscr{E}$ ein $\mathcal{O}_{X \times S}$-Modul. Für alle $n \in \mathbb{Z}$ sei

$$
\mathscr{E}(n \infty):=\mathscr{E} \otimes_{\mathcal{O}_{X \times S}} \operatorname{pr}_{X}^{*} \mathcal{O}_{X}(n \infty)
$$

der Twist mit dem Geradenbündel $\operatorname{pr}_{X}^{*} \mathcal{O}_{X}(n \infty)$. Insbesondere ist

$$
\mathcal{O}_{X \times S}(n \infty) \cong \operatorname{pr}_{X}^{*} \mathcal{O}_{X}(n \infty) \text {. }
$$

\section{Bemerkung 2.55}

Für alle $n, m \in \mathbb{Z}$ gilt

$$
\mathcal{O}_{X \times S}(n \infty) \otimes_{\mathcal{O}_{X \times S}} \mathcal{O}_{X \times S}(m \infty) \cong \mathcal{O}_{X \times S}((n+m) \infty)
$$

und

$$
\mathscr{E}(n \infty) \otimes_{\mathcal{O}_{X \times S}} \mathcal{O}_{X \times S}(m \infty) \cong \mathscr{E}((n+m) \infty)
$$

\section{Satz 2.56}

Es gibt eine Zahl $m \in \mathbb{N}$, so dass $\mathcal{O}_{X \times S}(m \infty)$ ein sehr amples Geradenbündel ist.

Beweis Nach Satz 2.30 gibt es eine Zahl $m \in \mathbb{N}$ und eine abgeschlossene Einbettung $f: X \longrightarrow \mathbb{P}_{K}^{n}$, so dass $\mathcal{O}_{X}(m \infty)=f^{*} \mathcal{O}_{\mathbb{P}_{K}^{n}}(1)$ gilt. Nach [Har77], Teil II, Aufgabe 3.11, Seite 92, ist auch

$$
f \times \operatorname{id}_{S}: X \times S \longrightarrow \mathbb{P}_{K}^{n} \times S=\mathbb{P}_{S}^{n}
$$

eine abgeschlossene Einbettung. Es folgt

$$
\left(f \times \operatorname{id}_{S}\right)^{*} \mathcal{O}_{\mathbb{P}_{S}^{n}}(1)=\left(f \times \operatorname{id}_{S}\right)^{*} \operatorname{pr}_{\mathbb{P}_{K}^{n}}^{*} \mathcal{O}_{\mathbb{P}_{K}^{n}}(1)=\operatorname{pr}_{X}^{*} f^{*} \mathcal{O}_{\mathbb{P}_{K}^{n}}(1)=\operatorname{pr}_{X}^{*} \mathcal{O}_{X}(m \infty)
$$

\section{Lemma 2.57}

Sei $S=\operatorname{spec} R$ ein affines Schema und sei $\mathscr{E}$ ein $\mathcal{O}_{X \times S^{-}}$Vektorbündel von endlichem Rang. Dann gibt es $k, r \in \mathbb{Z}$ und ein $\mathcal{O}_{X \times S}$-Vektorbündel $\mathscr{F}$ von endlichem Rang, so dass es eine exakte Sequenz der Form

$$
0 \longrightarrow \mathscr{F} \longrightarrow \mathcal{O}_{X \times S}(k \infty)^{\oplus r} \longrightarrow \mathscr{E} \longrightarrow 0
$$

gibt. 
Beweis Nach Satz 2.56 ist für geeignetes $m \in \mathbb{Z}$ das Vektorbündel $\mathcal{O}_{X \times S}(m \infty)$ sehr ampel. Nach [Liu02], Kapitel 5, Theorem 1.27, Seite 167, gibt es eine Zahl $n \in \mathbb{Z}$, so dass $\mathscr{E}(n m \infty)$ von globalen Schnitten erzeugt wird, das heißt, es gibt für ein geeignetes $r \in \mathbb{N}$ eine surjektive Abbildung

$$
\mathcal{O}_{X \times S}^{\oplus r} \longrightarrow \mathscr{E}(n m \infty) \longrightarrow 0 .
$$

Tensorieren der Sequenz mit $\mathcal{O}_{X \times S}(-n m \infty)$ ergibt

$$
\mathcal{O}_{X \times S}((-n m) \infty)^{\oplus r} \longrightarrow \mathscr{E} \longrightarrow 0 .
$$

Nach Lemma 2.44 ist auch der Kern dieser Abbildung, wir nennen ihn $\mathscr{F}$, ein Vektorbündel von endlichem Rang.

\section{Lemma 2.58}

Sei $S=\operatorname{spec} R$ ein affines Schema und sei $\mathscr{E}$ ein $\mathcal{O}_{X \times S}$-Vektorbündel von endlichem Rang. Dann gibt es Zahlen $m, n \in \mathbb{Z}$ mit $H^{0}(X \times S, \mathscr{E}(n \infty)) \neq 0$ und $H^{0}(X \times S, \mathscr{E}(m \infty))=0$.

\section{Beweis}

a) Da $\mathcal{O}_{X \times S}(k \infty)$ für ein geeignetes $k \in \mathbb{N}$ ein sehr amples Geradenbündel ist, gibt es ein $n^{\prime} \in \mathbb{N}$, so dass $\mathscr{E}\left(n^{\prime} k \infty\right)$ von globalen Schnitten erzeugt wird. Insbesondere für $n:=n^{\prime} k$ ist $H^{0}(X \times S, \mathscr{E}(n \infty)) \neq 0$.

b) Für $k<0$ hat $\mathcal{O}_{X}(k \infty)$ keine globalen Schnitte. Da $R / K$ ein flacher Basiswechsel ist, gilt für $k<0$

$$
H^{0}\left(X \times S, \mathcal{O}_{X \times S}(k \infty)\right) \cong H^{0}\left(X, \mathcal{O}_{X}(k \infty)\right) \otimes_{K} R=0 .
$$

c) Sei $\mathscr{E}^{\vee}$ das duale Vektorbündel. Nach Lemma 2.57 gibt es Zahlen $n^{\vee}, r \in$ $\mathbb{N}$, so dass es eine exakte Sequenz der Form

$$
\mathcal{O}_{X \times S}\left(-n^{\vee} \infty\right)^{\oplus r} \longrightarrow \mathscr{E}^{\vee} \longrightarrow 0
$$

gibt. Dualisierung ergibt die Sequenz

$$
0 \longrightarrow \mathscr{E} \longrightarrow \mathcal{O}_{X \times S}\left(n^{\vee} \infty\right)^{\oplus r} .
$$

Wir wählen $m<-n^{\vee}$. Dann liefert Tensorieren mit $\mathcal{O}_{X \times S}(m \infty)$

$$
0 \longrightarrow \mathscr{E}(m \infty) \longrightarrow \mathcal{O}_{X \times S}\left(\left(n^{\vee}+m\right) \infty\right)^{\oplus r} \text {. }
$$

Da $\mathcal{O}_{X \times S}\left(\left(n^{\vee}+m\right) \infty\right)$ nach Wahl von $m$ keine globalen Schnitte mehr hat, gilt dies auch für $\mathscr{E}(m \infty)$. 
Sei $\mathscr{E}$ ein $\mathcal{O}_{X \times S^{-}}$Vektorbündel von Rang $d / \operatorname{deg}(\infty)$ und sei für alle $i \in \mathbb{Z}$

$$
\mathscr{E}_{i}:=\mathscr{E}(i \infty)
$$

Im Folgenden zeigen wir, dass für alle $i \in \mathbb{Z}$ die $\mathcal{O}_{S}$-Moduln $\operatorname{pr}_{S *} \mathscr{E}_{i} / \mathscr{E}_{i-1}$ Vektorbündel von Rang $d$ sind.

\section{Lemma 2.59}

Für alle $i \in \mathbb{Z}$ ist

$$
\mathscr{E}_{i} / \mathscr{E}_{i-1} \cong \mathscr{E} \otimes_{\mathcal{O}_{X \times S}} \mathcal{O}_{X \times S}(i \infty) / \mathcal{O}_{X \times S}((i-1) \infty)
$$

Beweis Tensorieren der exakten Sequenz

$$
\begin{aligned}
0 \longrightarrow \mathcal{O}_{X \times S}((i-1) \infty) \longrightarrow \mathcal{O}_{X \times S}(i \infty) \longrightarrow \\
\longrightarrow \mathcal{O}_{X \times S}(i \infty) / \mathcal{O}_{X \times S}((i-1) \infty) \longrightarrow 0
\end{aligned}
$$

mit $\mathscr{E}$ liefert die Behauptung.

\section{Lemma 2.60}

Für alle $i \in \mathbb{Z}$ ist

$$
\mathcal{O}_{X \times S}(i \infty) / \mathcal{O}_{X \times S}((i-1) \infty) \cong \operatorname{pr}_{X}^{*} \mathcal{O}_{X}(i \infty) / \mathcal{O}_{X}((i-1) \infty)
$$

Beweis Der Funktor $\operatorname{pr}_{X}^{*}$ ist ein exakter Funktor, da $\mathcal{O}_{X \times S}$ ein flacher $\mathcal{O}_{X^{-}}$ Modul ist. Anwendung des Funktors auf die Sequenz

$$
0 \longrightarrow \mathcal{O}_{X}((i-1) \infty) \longrightarrow \mathcal{O}_{X}(i \infty) \longrightarrow \mathcal{O}_{X}(i \infty) / \mathcal{O}_{X}((i-1) \infty) \longrightarrow 0
$$

liefert die Behauptung.

\section{Bemerkung 2.61}

Das zur Idealgarbe $\mathcal{O}_{X}(-\infty)$ gehörende abgeschlossene Unterschema ist spec $k(\infty) \hookrightarrow X$. Da $\mathcal{O}_{X}(-\infty)$ ein flacher $\mathcal{O}_{X}$-Modul ist, folgt, dass $\mathcal{O}_{X \times S}$ die Idealgarbe zum abgeschlossenen Unterschema $k(\infty) \times S \hookrightarrow X \times S$ ist.

Lemma 2.62

Sei $\iota: \operatorname{spec} k(\infty) \longrightarrow X$ die kanonische Einbettung. Dann gilt für alle $i \in \mathbb{Z}$

$$
\mathcal{O}_{X}(i \infty) / \mathcal{O}_{X}((i-1) \infty) \cong \iota_{*} k(\infty) .
$$

Beweis Die Idealgarbe $\mathcal{O}_{X}(-\infty)$ annuliert für alle $i \in \mathbb{Z}$ den $\mathcal{O}_{X}$-Modul $\mathcal{O}_{X}(i \infty) / \mathcal{O}_{X}((i-1) \infty)$. Außerdem gilt $\iota^{*} \mathcal{O}_{X}(i \infty) / \mathcal{O}_{X}((i-1) \infty) \cong k(\infty)$. Die Behauptung ergibt sich nun aus Lemma 2.22, 3). 


\section{Folgerung 2.63}

Sei $\iota_{S}: \operatorname{spec} k(\infty) \times S \longrightarrow X \times S$. Für alle $i \in \mathbb{Z}$ ist

$$
\mathcal{O}_{X \times S}(i \infty) / \mathcal{O}_{X \times S}((i-1) \infty) \cong \iota_{S *} \mathcal{O}_{k(\infty) \times S} .
$$

Beweis Mit $S \longrightarrow$ spec $K$ ist auch die Abbildung $X \times S \longrightarrow X$ flach. Die Aussage folgt jetzt aus Lemma 2.23, 1).

\section{Lemma 2.64}

Sei $\iota_{S}: \operatorname{spec} k(\infty) \times S \longrightarrow X \times S$. Für alle $i \in \mathbb{Z}$ gilt

$$
\mathscr{E}_{i} / \mathscr{E}_{i-1} \cong \iota_{S *} \iota_{S}^{*} \mathscr{E}_{i} / \mathscr{E}_{i-1}
$$

Beweis Nach Bemerkung 2.61 ist die Idealgarbe zum abgeschlossenen Unterschema spec $k(\infty) \times S$ gegeben durch $\mathcal{O}_{X \times S}(-\infty)$. Es gilt

$$
\mathscr{E}_{i-1} \cong \mathscr{E}_{i} \otimes_{\mathcal{O}_{X \times S}} \mathcal{O}_{X \times S}(-\infty) \cong \mathcal{O}_{X \times S}(-\infty) \mathscr{E}_{i}
$$

Insbesondere annuliert die Idealgarbe $\mathcal{O}_{X \times S}(-\infty)$ den Modul $\mathscr{E}_{i} / \mathscr{E}_{i-1}$. Die Behauptung folgt nun aus Lemma 2.22, 3).

\section{Lemma 2.65}

Für alle $i \in \mathbb{Z}$ ist

$$
\iota_{S}^{*} \mathscr{E}_{i} / \mathscr{E}_{i-1} \cong \iota_{S}^{*} \mathscr{E}
$$

Beweis Da das Diagramm

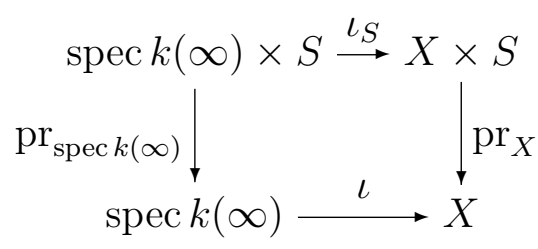

kommutiert, folgt

$$
\begin{aligned}
\iota_{S}^{*}\left(\mathscr{E}_{i} / \mathscr{E}_{i-1}\right) & \cong \iota_{S}^{*}\left(\mathscr{E} \otimes_{\mathcal{O}_{X \times S}} \operatorname{pr}_{X}^{*} \iota_{*} k(\infty)\right) \quad(\operatorname{vgl} . \text { Lemma 2.59, 2.60, 2.62) } \\
& \cong \iota_{S}^{*} \mathscr{E} \otimes_{\mathcal{O}_{\text {spec } k(\infty) \times S}} \iota_{S}^{*} \operatorname{pr}_{X}^{*} \iota_{*} k(\infty) \\
& \cong \iota_{S}^{*} \mathscr{E} \otimes_{\mathcal{O}_{\text {spec } k(\infty) \times S}} \operatorname{pr}_{\mathrm{spec} k(\infty)}^{*} \iota^{*} \iota_{*} k(\infty) \\
& \cong \iota_{S}^{*} \mathscr{E} \otimes_{\mathcal{O}_{\text {spec } k(\infty) \times S}} \operatorname{pr}_{\mathrm{spec} k(\infty)}^{*} k(\infty) \quad(\text { vgl. Lemma 2.22, 1) } \\
& \cong \iota_{S}^{*} \mathscr{E} .
\end{aligned}
$$

Folgerung 2.66

Für alle $i \in \mathbb{Z}$ ist $\operatorname{pr}_{S *} \mathscr{E}_{i} / \mathscr{E}_{i-1}$ ein $\mathcal{O}_{S}$-Vektorbündel von Rang d. 
Beweis Nach Lemma 2.64 gilt

$$
\operatorname{pr}_{S *} \mathscr{E}_{i} / \mathscr{E}_{i-1} \cong \operatorname{pr}_{S *} \iota_{S *} \iota_{S}^{*} \mathscr{E}_{i} / \mathscr{E}_{i-1}
$$

Es reicht deshalb, die Aussage für $\iota_{S}^{*}\left(\mathscr{E}_{i} / \mathscr{E}_{i-1}\right)$ zu zeigen. Als Pullback des Vektorbündels $\mathscr{E}$ ist $\iota_{S}^{*}\left(\mathscr{E}_{i} / \mathscr{E}_{i-1}\right)$ ein $\mathcal{O}_{\text {spec } k(\infty) \times S^{-}}$Vektorbündel von Rang $\frac{d}{\operatorname{deg}(\infty)}$. Die endliche Körpererweiterung $k(\infty) / k$ hat den Grad $\operatorname{deg}(\infty)$. Daraus ergibt sich, dass $\operatorname{pr}_{S *} \iota_{S}^{*}\left(\mathscr{E}_{i} / \mathscr{E}_{i-1}\right)$ ein $\mathcal{O}_{S^{-}}$Vektorbündel von Rang $d$ ist.

\section{Lemma 2.67}

Sei $S=\operatorname{spec} R$ ein affines Schema und sei $\mathscr{E}$ ein $\mathcal{O}_{X \times S^{-}}$Vektorbündel. Dann ist $H^{1}\left(X \times S, \mathscr{E}_{i} / \mathscr{E}_{i-1}\right)=0$ für alle $i \in \mathbb{Z}$.

Beweis Da $S$ ein affines Schema ist, hat $\mathscr{E}_{i} / \mathscr{E}_{i-1}$ einen affinen Träger (vgl. Lemma 2.64). Es folgt die Behauptung (vgl. [Liu02], Kapitel 5, Aufgabe 2.3 (b), Seite 191 und Theorem 2.18, Seite 186).

\section{Folgerung 2.68}

Sei $S=\operatorname{spec} R$ ein affines Schema und sei $\mathscr{E}$ ein $\mathcal{O}_{X \times S^{-}}$Vektorbündel. Dann gibt es eine Zahl $n_{0} \in \mathbb{Z}$ mit $H^{0}(X \times S, \mathscr{E}(i \infty))=0$ für $i \leq n_{0}$ und $H^{0}(X \times$ $S, \mathscr{E}(i \infty)) \neq 0$ für $i>n_{0}$.

Beweis Aus der exakten Sequenz

$$
0 \longrightarrow \mathscr{E}_{i} \longrightarrow \mathscr{E}_{i+1} \longrightarrow \mathscr{E}_{i+1} / \mathscr{E}_{i} \longrightarrow 0
$$

erhalten wir die lange exakte Kohomologiesequenz

$$
\begin{aligned}
0 \longrightarrow H^{0}\left(X \times S, \mathscr{E}_{i}\right) \longrightarrow H^{0}\left(X \times S, \mathscr{E}_{i+1}\right) \longrightarrow H^{0}\left(X \times S, \mathscr{E}_{i} / \mathscr{E}_{i+1}\right) \longrightarrow \\
\longrightarrow H^{1}\left(X \times S, \mathscr{E}_{i}\right) \longrightarrow H^{1}\left(X \times S, \mathscr{E}_{i+1}\right) \longrightarrow 0
\end{aligned}
$$

Aus der Injektivität der ersten Abbildung folgt mit Lemma 2.58 die Behauptung.

\section{Satz 2.69}

Sei $S=\operatorname{spec} R$ ein affines Schema und sei $\mathscr{E}$ ein Vektorbündel auf $X \times S$. Sei $U:=X \backslash\{\infty\}$. Für alle $n \in \mathbb{Z}$ seien

$$
H^{0}(X \times S, \mathscr{E}(n \infty)) \longrightarrow H^{0}(U \times S, \mathscr{E}(n \infty))=H^{0}(U \times S, \mathscr{E})
$$

die kanonischen Abbildungen, und für alle $n, m \in \mathbb{Z}, n \leq m$, seien

$$
H^{0}(X \times S, \mathscr{E}(n \infty)) \longrightarrow H^{0}(X \times S, \mathscr{E}(m \infty))
$$

die kanonischen Inklusionen. Dann gilt

$$
\underset{n}{\lim _{n}} H^{0}(X \times S, \mathscr{E}(n \infty)) \cong H^{0}(U \times S, \mathscr{E}) .
$$


Beweis Die Aussage ist mit flachem Basiswechsel bezüglich des Schemas $S$ verträglich, sei deshalb ohne Einschränkung der Allgemeinheit $R=K$. Die Behauptung ergibt sich nun direkt aus Satz 2.31.

\section{Satz 2.70}

Sei $S=\operatorname{spec} R$ ein affines Schema und sei $\mathscr{E}$ ein Vektorbündel auf $X \times S$. Sei $U:=X \backslash\{\infty\}$. Dann gilt

$$
\left.H^{0}\left(X \times S, \underset{n}{\lim _{n}} \mathscr{E}_{n}\right)\right)=\underset{n}{\lim } H^{0}\left(X \times S, \mathscr{E}_{n}\right)=H^{0}(U \times S, \mathscr{E}) .
$$

Beweis Sei $\mathscr{F}:=\lim _{n} \mathscr{E}_{n}$. Mit $X$ und $S$ ist auch $X \times S$ separiert und quasi-kompakt. Insbesondere gibt es eine endliche, affine, offene Überdeckung $X=\cup_{i=1}^{k} U_{i}$. Wir können deshalb die Kohomologie des quasi-kohärenten $\mathcal{O}_{X \times S^{-}}$Moduln $\mathscr{F}$ mit Hilfe des Čech-Komplexes berechnen. Wir erhalten die exakte Sequenz

$$
0 \longrightarrow H^{0}(X \times S, \mathscr{F}) \longrightarrow \prod_{i=1}^{k} H^{0}\left(U_{i}, \mathscr{F}\right) \longrightarrow \prod_{i, j=1}^{k} H^{0}\left(U_{i j}, \mathscr{F}\right)
$$

wobei wir $U_{i j}:=U_{i} \cap U_{j}$ setzen. Wir zeigen, dass die kanonische Abbildung

$$
u: \underset{n}{\lim _{n}} H^{0}\left(X \times S, \mathscr{E}_{n}\right) \longrightarrow H^{0}(X \times S, \mathscr{F})
$$

ein Isomorphismus ist.

Zum Beweis der Injektivität sei $\left(s_{n}\right) \in \lim _{\longrightarrow} H^{0}\left(X \times S, \mathscr{E}_{n}\right)$ mit $u\left(\left(s_{n}\right)\right)=0$ gegeben. Dann gibt es für jedes $1 \leq i \leq \vec{k}$ eine Zahl $n_{i}$ mit $\left.s_{n}\right|_{U_{i}}=0$ für alle $n \geq n_{i}$. Sei $n^{\prime}:=\max _{i} n_{i}$. Dann ist $\left.s_{n}\right|_{U_{i}}=0$ für alle $1 \leq i \leq k$ und $n \geq n^{\prime}$, und es ist $\left(s_{n}\right)=0$.

Zum Beweis der Surjektivität sei $s \in H^{0}(X \times S, \mathscr{F})$ gegeben. Dann gibt es für jedes $1 \leq i \leq k$ Elemente $s_{i} \in H^{0}\left(U_{i}, \mathscr{F}\right)$ mit $\left.s\right|_{U_{i}}=s_{i}$ und $\left.s_{i}\right|_{U_{i j}}=\left.s_{j}\right|_{U_{i j}}$ für alle $1 \leq i, j \leq k$.

Für jedes $1 \leq i \leq k$ gibt es $n_{i}$ mit $s_{i} \in H^{0}\left(U_{i}, \mathscr{E}_{n}\right)$ für alle $n \geq n_{i}$. Sei $n^{\prime \prime}:=\max _{i} n_{i}$. Dann ist $s_{i} \in H^{0}\left(U_{i}, \mathscr{E}_{n}\right)$ für $1 \leq i \leq k$ und $n \geq n^{\prime \prime}$.

Für alle $1 \leq i, j \leq k$ gibt es nun Zahlen $m_{i j} \geq n^{\prime \prime}$ mit

$$
\left.s_{i}\right|_{U_{i j}}=\left.s_{j}\right|_{U_{i j}} \in H^{0}\left(U_{i}, \mathscr{E}_{n}\right)
$$

für alle $n \geq m_{i j}$. Sei $m^{\prime}:=\max _{i, j} m_{i j}$. Dann gibt es ein Element $s \in H^{0}(X \times$ $\left.S, \mathscr{E}_{n}\right)$ mit $\left.s\right|_{U_{i}}=s_{i}$ für jedes $1 \leq i \leq k$ und alle $n \geq m^{\prime}$. Es folgt die Behauptung.

\section{Bemerkung 2.71}

Satz 2.70 gilt für alle quasi-kompakten, separierten Schemata und induktive, filtrierte Systeme von quasi-kohärenten Moduln. Eine allgemeine Version des Satzes findet sich in [GD66], Théoréme (8.5.2). 


\subsection{Konstruktion von Vektorbündeln auf $X \times S$}

\section{Satz 2.72}

Ein Vektorbündel von Rang d auf $X \times S$ lässt sich durch die folgenden Daten konstruieren:

- $\mathscr{M}$ : Vektorbündel von Rangd auf $\operatorname{spec} A \times S$

- $\mathscr{E}_{\infty}$ : Vektorbündel von Rang d auf $\operatorname{spec} \mathcal{O}_{X, \infty} \times S$

- Isomorphismus: $\mathscr{M} \otimes_{A} F \cong \mathscr{E}_{\infty} \otimes_{\mathcal{O}_{X, \infty}} F$

Sei $P \neq \infty$ ein abgeschlossener Punkt von $X$. Nach Satz 2.27 bildet dann $U:=X \backslash\{\infty\}=\operatorname{spec} A$ und $V:=X \backslash\{P\}=\operatorname{spec} B$ eine offene, affine Überdeckung von $X=U \cup V$.

\section{Lemma 2.73}

In der obigen Notation gilt:

1) $\operatorname{spec} \mathcal{O}_{X, \infty} \times_{X} U \cong \operatorname{spec} F$

2) $\operatorname{spec} \mathcal{O}_{X, \infty} \times_{X} \operatorname{spec} \mathcal{O}_{X, \infty} \cong \operatorname{spec} \mathcal{O}_{X, \infty}$

3) $\operatorname{spec} A \times_{X} \operatorname{spec} A \cong \operatorname{spec} A$

\section{Beweis}

1) Das Faserprodukt $\operatorname{spec} \mathcal{O}_{X, \infty} \times_{X} U$ lässt sich beschreiben durch die Überdeckung von $X$ durch $U=\operatorname{spec} A$ und $V=\operatorname{spec} B$, d.h.:

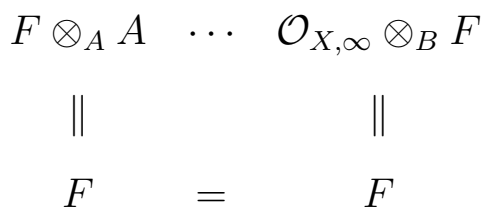

Dies zeigt 1).

2) Wie in 1) liefert die Überdeckung von $X$ durch $U$ und $V$

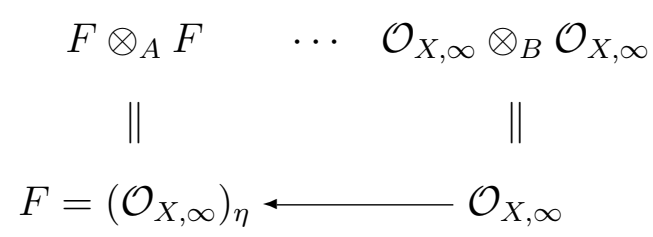


und

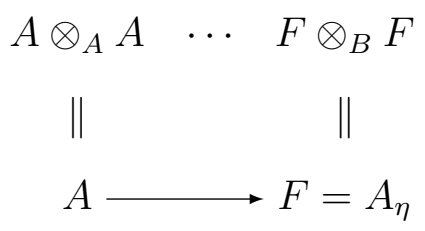

dies zeigt 2) und 3).

Beweis (des Satzes) Nach Aussage 2. des Lemmas ist spec $F$ das Faserprodukt von spec $A$ mit spec $\mathcal{O}_{X, \infty}$ über $X$. Basiswechsel ergibt das folgende Diagramm:

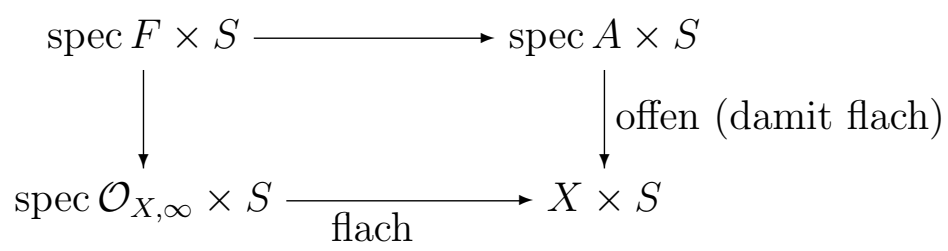

Weiterhin folgt mit 2. und 3. des Lemmas, dass die Bedingungen für den treuflachen Abstieg erfüllt sind (vgl. [Wat79] 17.2). Es folgt die Behauptung.

\subsection{Frobenius-Twist von Garben}

Sei $S$ ein beliebiges Schema über dem endlichen Körper $\mathbb{F}_{q}$. Der arithmetische Frobenius-Endomorphismus Frob $_{S}^{a}: S \longrightarrow S$ ist wie folgt definiert:

$$
\begin{aligned}
S \stackrel{\text { id }}{\longrightarrow} S \text { und } \mathcal{O}_{S}(U) & \longrightarrow \mathcal{O}_{S}(U) \text { für alle } U \subset X \text { offen } \\
f & \longmapsto f^{q} .
\end{aligned}
$$

Sei $X$ ein Schema über $S$. Das kommutative Diagramm

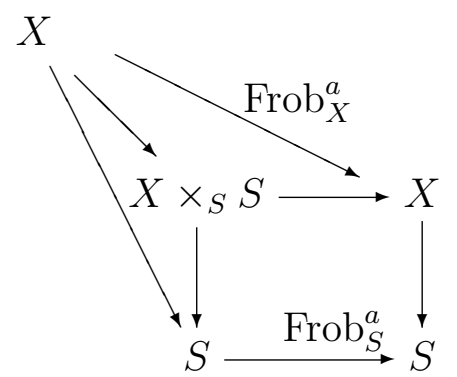

bestimmt den Pullback Frob $_{S}^{*} X:=X \times_{S} S$ und einen Morphismus, den geometrischen Frobenius-Morphismus Frob ${ }^{g}: X \longrightarrow$ Frob $_{S}^{*} X$ von Schemata über $S$. Im Gegensatz zum geometrischen Frobenius-Endomorphismus ist der arithmetische Frobenius-Endomorphismus im Allgemeinen kein $S$ Morphismus (vgl. [Har77], Teil IV, Kapitel 2, Seite 301 f). 


\section{Definition 2.74}

1) Sei $R$ eine $\mathbb{F}_{q}$-Algebra und seien $M, N$ beliebige $R$-Moduln. Eine additive Abbildung $f: M \longrightarrow N$ heißt $q$-linear, falls für alle $r \in R$ und $m \in M$

$$
f(r m)=r^{q} f(m)
$$

gilt.

2) Seien S,T Schemata über $\mathbb{F}_{q}$. Sei $\mathscr{E}$ eine Modulgarbe auf $T \times S$. Man definiert den Frobenius-Twist von $\mathscr{E}$ (bezüglich $S$ ) als

$$
\tau_{\mathscr{E}}:=\left(\mathrm{id}_{T} \times \operatorname{Frob}_{S}^{a}\right)^{*} \mathscr{E} .
$$

3) Seien $\mathscr{E}$, $\mathscr{F}$ zwei $\mathcal{O}_{T \times S}-$ Modulgarben.

Eine additive Abbildung $\mathscr{E} \longrightarrow \mathscr{F}$ heißt q-linear in $\mathcal{O}_{S}$, linear in $\mathcal{O}_{T}$, falls $\operatorname{pr}_{S *} \mathscr{E} \longrightarrow \operatorname{pr}_{S *} \mathscr{F}$ eine $\mathcal{O}_{S}$-q-lineare Abbildung und $\mathrm{pr}_{T *} \mathscr{E} \longrightarrow \mathrm{pr}_{T *} \mathscr{F}$ eine $\mathcal{O}_{T}$-lineare Abbildung ist. Im Folgenden sei dies der Einfachheit halber immer nur kurz mit $\mathcal{O}_{S}$-q-linear bezeichnet.

\section{Bemerkung 2.75}

Es gibt eine kanonische $q$-lineare Abbildung $\mathscr{E} \longrightarrow{ }^{\tau} \mathscr{E}$. Sei $U \subseteq T \times S$ eine offene, affine Teilmenge und $s \in \mathscr{E}(U)$. Dann definieren wir

$$
\begin{gathered}
\mathscr{E}(U) \longrightarrow \mathcal{O}_{T \times S}(U) \otimes_{\mathcal{O}_{T \times S}(U)} \mathscr{E}(U) \\
s \longrightarrow 1 \otimes s .
\end{gathered}
$$

\section{Lemma 2.76}

Sei $S=\operatorname{spec} K$ ein Körper. Dann ist die kanonische Abbildung $\mathscr{E} \longrightarrow{ }^{\tau} \mathscr{E}$ injektiv.

Beweis Sei $U=\operatorname{spec} R \otimes K \subseteq T \times S$ eine affine, offene Teilmenge. Der Basiswechsel Frob $: K \longrightarrow K$ ist, da $K$ ein Körper ist, flach und es gilt $\tau_{\mathscr{E}}(U) \cong K \otimes_{K} \mathscr{E}(U)$. Tensorieren der injektiven Abbildung Frob $: K \longrightarrow K$ mit $\mathscr{E}(U)$ liefert die Behauptung.

\section{Bemerkung 2.77}

Eine additive Abbildung $\mathscr{E} \longrightarrow \mathscr{F}$ von $\mathcal{O}_{T \times S^{-}}$Modulgarben ist genau dann $\mathcal{O}_{T \times S^{-}}$linear, wenn $\operatorname{pr}_{S *} \mathscr{E} \longrightarrow \operatorname{pr}_{S *} \mathscr{F}$ eine $\mathcal{O}_{S}$-lineare und $\operatorname{pr}_{T *} \mathscr{E} \longrightarrow \operatorname{pr}_{T *} \mathscr{F}$ eine $\mathcal{O}_{T}$-lineare Abbildung ist. 
Satz 2.78

Seien $\mathscr{E}, \mathscr{F}$ Modulgarben auf $T \times S$ und sei $f: \mathscr{E} \longrightarrow \mathscr{F}$ eine $\mathcal{O}_{S}-q$-lineare Abbildung. Dann gibt es genau eine $\mathcal{O}_{T \times S}$ lineare Abbildung ${ }^{\tau} f:{ }^{\tau} \mathscr{E} \longrightarrow \mathscr{F}$, so dass das Diagramm

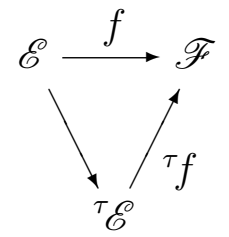

kommutiert.

\section{Beweis}

1) Für eine offene, affine Teilmenge $U \subseteq T \times S$ definieren wir

$$
\begin{aligned}
\mathcal{O}_{T \times S}(U) \otimes_{\mathcal{O}_{T \times S}(U)} \mathscr{E}(U) & \longrightarrow \mathscr{F}(U) \\
x \otimes s & \longmapsto x f(s) .
\end{aligned}
$$

Diese Abbildung ist additiv und linear in $\mathcal{O}_{S}$ und $\mathcal{O}_{T}$, also $\mathcal{O}_{T \times S^{-}}$linear.

2) Die Abbildung ${ }^{\tau} f$ ist auf dem Bild von $\mathscr{E}(U)$ in ${ }^{\tau} \mathscr{E}(U)$ durch das kommutative Diagramm bereits festgelegt. Da ${ }^{\tau} \mathscr{E}(U)$ von diesem Bild erzeugt wird, ist die Abbildung eindeutig festgelegt. 


\section{Drinfeld-Moduln und ihre Vektorbündel}

\subsection{Drinfeld-Moduln}

Sei $X / \mathbb{F}_{q}$ eine glatte, absolut irreduzible Kurve, $F:=F(X)$ der Funktionenkörper von $X$ und $\infty \in X$ ein abgeschlossener Punkt. Sei $\mathcal{O}_{X, P}$ der lokale Ring im Punkt $P \in X$, das zugehörige maximale Ideal sei $\mathfrak{m}_{X, P}$ und $k(P):=\mathcal{O}_{X, P} / \mathfrak{m}_{X, P}$ der Restekörper. Mit $\operatorname{deg}(P):=\left[k(P): \mathbb{F}_{q}\right]$ sei der Grad der Restekörpererweiterung bezeichnet. Aufgrund der Glattheit der Kurve $X$ sind die lokalen Ringe $\mathcal{O}_{X, P}$ diskrete Bewertungsringe. Die zugehörige Bewertung und ihre kanonische Fortsetzung auf dem Funktionenkörper $F$ sei mit $v_{P}$, oder, falls keine Missverständnisse zu befürchten sind, mit $P$ bezeichnet. Der Ring

$$
A:=\Gamma\left(X \backslash \infty, \mathcal{O}_{X}\right)=\left\{x \in F \mid v_{P}(x) \geq 0 \forall P \in X \backslash \infty\right\}
$$

ist ein Dedekindring. Für alle $x \in F$ gilt die Produktformel

$$
\sum_{P \in X} \operatorname{deg}(P) v_{P}(x)=0 .
$$

Es folgt, dass $\infty(a) \leq 0$ für alle $a \in A$ gilt. Wir führen aus diesem Grund die Bezeichnung deg $a:=-\infty(a)$ für alle Elemente $a \in F$ ein.

Sei $S / \mathbb{F}_{q}$ ein Schema, $\mathscr{L}$ ein Geradenbündel über $S$ und sei $\mathbb{G}_{a / \mathscr{L}}$ das additive Gruppenschema zum Geradenbündel $\mathscr{L}$. Für alle offenen Teilmengen $U \subset S$ ist das Gruppenschema gegeben durch

$$
\mathbb{G}_{a / \mathscr{L}}(U)=\mathscr{L}(U)
$$

Die (additiven) Gruppen $\mathscr{L}(U)$ sind in kanonischer Weise $\mathbb{F}_{q}$-Vektorräume.

\section{Satz 3.1}

1) Seien $\mathscr{L}$ und $\mathscr{M}$ Geradenbündel über $S$. Seien $\operatorname{Hom}_{\mathbb{F}_{q}}\left(\mathbb{G}_{a / \mathscr{L}}, \mathbb{G}_{a / \mathscr{M}}\right)$ die $\mathbb{F}_{q}$-linearen Endomorphismen der beiden zugehörigen Gruppenschemata. Sei $\mathscr{H}_{o m_{\mathbb{F}_{q}}}\left(\mathbb{G}_{a / \mathscr{L}}, \mathbb{G}_{a / \mathscr{M}}\right)$ die assoziierte Homomorphismen-Garbe über $S$. Dann gilt

$$
\mathscr{H} o m_{\mathbb{F}_{q}}\left(\mathbb{G}_{a / \mathscr{L}}, \mathbb{G}_{a / \mathscr{M}}\right) \cong \bigoplus_{n=0}^{\infty} \mathscr{M} \otimes_{\mathcal{O}_{S}} \mathscr{L}^{-q^{n}} .
$$

2) Sei $\operatorname{End}_{\mathbb{F}_{q}}\left(\mathbb{G}_{a / \mathscr{L}}\right)$ der Endomorphismenring der $\mathbb{F}_{q}$-linearen Endomorphismen des Gruppenschemas $\mathbb{G}_{a / \mathscr{L}}$ und sei $\mathscr{E} n d_{\mathbb{F}_{q}}\left(\mathbb{G}_{a / \mathscr{L}}\right)$ die assoziierte Endomorphismen-Garbe über $S$. Es gilt

$$
\mathscr{E} n d_{\mathbb{F}_{q}}\left(\mathbb{G}_{a / \mathscr{L}}\right) \cong \bigoplus_{n=0}^{\infty} \mathscr{L}^{1-q^{n}}
$$


Beweis Siehe [Leh00], Kapitel 1, Proposition 2.3, Seite 5.

\section{Folgerung 3.2}

Sei $U=\operatorname{spec} R \subseteq S$ eine affine, offene Trivialisierung des Geradenbündels $\mathscr{L}$. Dann gilt

$$
\mathscr{E} n d_{\mathbb{F}_{q}}\left(\mathbb{G}_{a / \mathscr{L}}\right)(U) \cong \operatorname{End}_{\mathbb{F}_{q}}\left(\mathbb{G}_{a / R}\right) \cong R\{\tau\} .
$$

\section{Bemerkung 3.3}

Eine gute Einführung in die Theorie der Schiefpolynomringe über einem Körper befindet sich in der Arbeit [Thi01], Abschnitt 2.3.

\section{Bemerkung 3.4}

In der Situation von Satz 3.1 sei $\varphi \in \operatorname{End}_{\mathbb{F}_{q}}\left(\mathbb{G}_{a / \mathscr{L}}\right)$ ein endlicher Morphismus. Dann ist für alle $s \in S$ der Rang (rk) von $\varphi$ wohldefiniert. Der Rang von $\varphi$ ist auf jeder Zusammenhangskomponente von $S$ konstant (vgl. [Leh00], Kapitel 1, Proposition 2.6, Seite 6).

\section{Definition 3.5 ([Dri76])}

Sei char $: S \longrightarrow$ spec $A$ ein Morphismus über $\mathbb{F}_{q}$. Ein Drinfeld-Modul $E:=\left(\mathbb{G}_{a / \mathscr{L}}, e\right)$ besteht aus einem additiven Gruppenschema $\mathbb{G}_{a / \mathscr{L}}$ und einem Ringhomomorphismus e $: A \longrightarrow \operatorname{End}_{\mathbb{F}_{q}}\left(\mathbb{G}_{a / \mathscr{L}}\right)$ mit:

1) Der Morphismus e $(a)$ ist endlich für alle $a \in A$ und für alle Punkte von $S$ gibt es ein $a \in A$, so dass rk $e(a)>1$ ist.

2) Das Diagramm

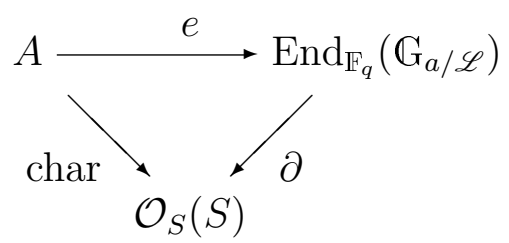

kommutiert.

\section{Satz 3.6}

1) Sei S ein zusammenhängendes Schema. Dann gibt es eine natürliche Zahl $d>0$ mit

$$
\operatorname{rk} e(a)=-d \operatorname{deg}(\infty) \infty(a) .
$$

Die Zahl d heißt Rang des Drinfeld-Moduls. Ist der Rang eines DrinfeldModuls auf allen Zusammenhangskomponenten konstant d, dann sagen wir der Drinfeld-Modul hat Rang d. 
2) Morphismen von Drinfeld-Moduln $E=\left(\mathbb{G}_{a / \mathscr{L}}, e\right)$ und $F=\left(\mathbb{G}_{a / \mathscr{L}^{\prime}}, f\right)$ sind Homomorphismen von Gruppenschemata über $\mathbb{F}_{q}$

$$
u: E \longrightarrow F
$$

mit ue $(a)=f(a)$ u für alle $a \in A$, das heißt, das Diagramm

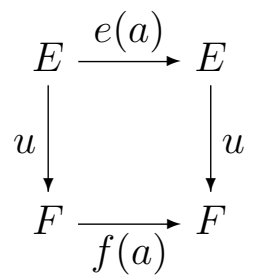

kommutiert.

3) Sei $E=\left(\mathbb{G}_{a / \mathscr{L}}, e\right)$ ein Drinfeld-Modul von Rang d. Dann gibt einen Isomorphismus u von Drinfeld-Moduln mit

$$
u e(a) u^{-1}=: f(a),
$$

so dass für jede affine, offene Trivialisierung spec $R \subseteq S$ von $E$

$$
f(a)=\sum_{i=0}^{-d \operatorname{deg}(\infty) \infty(a)} r_{i}(a) \tau^{i}
$$

gilt und der Leitkoeffizient $r_{-d \operatorname{deg}(\infty) \infty(a)}(a)$ eine Einheit in $R$ ist. Ein solcher Drinfeld-Modul heißt in Standardform. Fordert man zusätzlich $\partial u=1$, dann ist $u$ eindeutig bestimmt ([Leh00] Kapitel 1, 2.8, Kapitel 2, 2.3).

4) Ist $S=\operatorname{spec} R$ ein affines Schema und ist $\mathscr{L}$ trivial, dann ist

$$
\operatorname{End}_{\mathbb{F}_{q}}\left(\mathbb{G}_{a / \mathscr{L}}\right) \cong R\{\tau\}
$$

und wir erhalten das Diagramm:

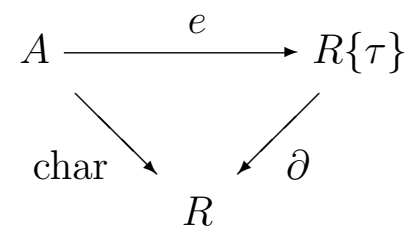

In diesem Fall wird der Drinfeld-Modul mit $(R, e)$ bezeichnet.

5) Sei mit $\operatorname{deg}_{\tau}$ der Grad eines Elements im Schiefpolynomring $R\{\tau\}$ bezeichnet. Dann gibt es für jedes Element $e(\tau) \in R\{\tau\}$ mit invertierbarem Leitkoeffizienten eine Rechtsdivision mit Rest

$$
F(\tau)=H(\tau) e(\tau)+R(\tau) \text { mit } \operatorname{deg}_{\tau} e(\tau)>\operatorname{deg}_{\tau} R(\tau) .
$$




\section{2 $\quad \mathbf{R} \otimes$ A-Modulstruktur auf $\mathbf{R}\{\tau\}$}

Sei $(R, e)$ ein Drinfeld-Modul über $A$. Auf $R\{\tau\}$ ist eine $R \otimes A$-ModulStruktur definiert durch

$$
(\lambda \otimes a) F(\tau):=\lambda F(\tau) e(a) .
$$

\section{Lemma 3.7}

Seien $(R, e)$ und $(R, f)$ Drinfeld-Moduln und $u:(R, e) \longrightarrow(R, f)$ ein Isomorphismus, dann sind die beiden $R \otimes A$-Modul-Strukturen auf $R\{\tau\}$ isomorph.

Beweis Der Isomorphismus $u$ ist gegeben durch ein invertierbares Element $u(\tau) \in R\{\tau\}$, insbesondere gibt es ein Element $u(\tau)^{-1} \in R\{\tau\}$ mit $u(\tau) u(\tau)^{-1}=1$. Sei

$$
\begin{aligned}
\varphi: R\{\tau\} & \longrightarrow R\{\tau\} \\
F(\tau) & \longmapsto F(\tau) u(\tau)^{-1}
\end{aligned}
$$

die durch Rechtsmultiplikation mit $u(\tau)^{-1}$ vermittelte Abbildung. Es folgt

$$
\begin{aligned}
\varphi((r \otimes a) F(\tau)) & =r F(\tau) e(a) u(\tau)^{-1}=r F(\tau) u(\tau)^{-1} f(a) \\
& =(r \otimes a) \varphi(F(\tau)) .
\end{aligned}
$$

\subsection{Projektivität von $\mathbf{R}\{\tau\}$}

\section{Satz 3.8}

Sei $(R, e)$ ein Drinfeld-Modul von Rang d über A. Dann ist der $R \otimes A$-Modul $R\{\tau\}$ endlich erzeugt und lokal frei von Rang $d$.

Aufgrund von Lemma 3.7 und Satz 3.3.3 reicht es, die Aussage für DrinfeldModuln in Standardform zu zeigen. Sei deshalb im Folgenden $(R, e)$ ein Drinfeld-Modul in Standardform.

Nach Definition gibt es ein $a \in A$ mit $\operatorname{deg}_{\tau}(e(a))>0$. Rechtsdivision mit Rest durch $e(a)$ im Ring $R\{\tau\}$ liefert ein Erzeugendensystem $1, \ldots, \tau^{\operatorname{deg}_{\tau}(e(a))-1}$ von $R\{\tau\}$ als $R \otimes A$-Modul. Insbesondere ist $R\{\tau\}$ ein endlich erzeugter $R \otimes A$-Modul.

Wir beweisen Satz 3.8 nun sukzessive für die folgenden fünf Fälle:

3.3.1 $R=L$ ist ein Körper und $A=\mathbb{F}_{q}[T]$.

3.3.2 $R=L$ ist ein Körper. 
3.3.3 $R$ ist ein reduzierter, noetherscher Ring.

3.3.4 $R$ ist ein noetherscher Ring.

3.3.5 $R$ ist ein beliebiger, kommutativer Ring.

\subsection{1 $R=L$ ist ein Körper und $A=\mathbb{F}_{\mathbf{q}}[\mathbf{T}]$}

In diesem Fall ist $L \otimes \mathbb{F}_{q}[T] \cong L[T]$ ein Hauptidealring. Ist $f(T) \in L[T]$ und $F(\tau) \in L\{\tau\}$, dann gilt

$$
\operatorname{deg}_{\tau}(f(T) F(\tau))=\operatorname{deg}_{\tau} F(\tau)+d \operatorname{deg}(f(T)) .
$$

Man zeigt, dass die Elemente $1, \ldots, \tau^{d-1}$ eine $L[T]$-Basis von $L\{\tau\}$ bilden. Rechtsdivision mit Rest durch $e(T)$ zeigt, dass die Elemente ein Erzeugendensystem bilden. Ist

$$
\sum_{i=0}^{d-1} f_{i}(T) \tau^{i}=0
$$

eine Darstellung der Null, dann gilt $\operatorname{deg}_{\tau}\left(f_{i}(T) \tau^{i}\right)=i+d \operatorname{deg}\left(f_{i}(T)\right)$. Da $0 \leq i<d$ gilt, ist nur die triviale Darstellung möglich.

\subsection{2 $\mathrm{R}=\mathrm{L}$ ist ein Körper}

Es gibt ein Element $T \in A$, so dass $F$ eine endliche, separable Körpererweiterung von $\mathbb{F}_{q}(T)$ und $A$ der ganze Abschluss von $\mathbb{F}_{q}[T]$ in $F$ ist (vgl. [Wie98], Abschnitt 2.2, Seite 5 und [Koc00], Abschnitt 5.1, Seite 142). Nach Satz 2.2, 1) und 4), Folgerung 2.26 und Satz 2.21 ist $L \otimes A$ der ganze Abschluss von $L[T]$ im Quotientenkörper $\operatorname{Quot}(L \otimes A)$.

Die Einschränkung des Drinfeld-Moduls über $A$ auf $\mathbb{F}_{q}[T]$ liefert einen Drinfeld-Modul über $\mathbb{F}_{q}$ von Rang $-d \operatorname{deg}(\infty) \infty(T)$ und der $L[T]$-Modul $L\{\tau\}$ ist nach 3.3.1 frei von diesem Rang.

Sei $m \in L\{\tau\}$ ein Torsionselement, das heißt, es gibt ein $y \in L \otimes A$ mit $y m=0$. Aus der Ganzheit von $y$ über $L[T]$ ergibt sich die Existenz einer Gleichung $\sum_{i=0}^{n-1} x_{i} y^{i}+y^{n}=0$ mit $x_{i} \in L[T]$. Da $L \otimes A$ ein Integritätsring ist, sei ohne Einschränkung $x_{0} \neq 0$. Es folgt:

$$
\begin{aligned}
0 & =-y^{n} m=\sum_{i=0}^{n-1} x_{i} y^{i} m \\
& =x_{0} m .
\end{aligned}
$$

Da die Operation von $L[T]$ auf $L\{\tau\}$ torsionsfrei ist, folgt $m=0$.

Nach Satz 2.5 und Satz 2.11 ist $L\{\tau\}$ ein projektiver Modul endlichen Ranges über $L \otimes A$. 
Für die Berechnung des Ranges reicht es, den Rang von

$$
[L \otimes A: L[T]]=\left[A: \mathbb{F}_{q}[T]\right]
$$

zu kennen, denn

$$
[L \otimes A: L[T]] \operatorname{rang}_{L \otimes A} L\{\tau\}=\operatorname{rang}_{L[T]} L\{\tau\} .
$$

Sei $\infty^{\prime}$ die Einschränkung der Bewertung $\infty$ auf $\mathbb{F}_{q}(T)$. Da $x(T) \geq 0$ für alle Bewertungen $x \neq \infty$, ist die Erweiterung unzerlegt in $\infty^{\prime}$, d.h. $\infty$ ist die einzige Bewertung über $\infty^{\prime}$. Nach der Fundamentalen Gleichung der algebraischen Zahlentheorie ([Neu92] Kapitel 2, Satz 8.5) gilt

$$
\left[F: \mathbb{F}_{q}(T)\right]=\left[A: \mathbb{F}_{q}[T]\right]=-\infty(T) \operatorname{deg}(\infty) .
$$

Es folgt die Behauptung.

\subsection{3 $R$ ist ein reduzierter, noetherscher Ring}

Verwendung findet das Projektivitäts-Kriterium Satz 2.7 für reduzierte Ringe.

Sei $\tilde{\mathfrak{p}} \in \operatorname{spec}(R \otimes A)$, sei $\tilde{\pi}: \operatorname{spec}(R \otimes A) \longrightarrow \operatorname{spec} R$ die kanonische Projektion und $\mathfrak{p}:=\tilde{\pi}(\tilde{\mathfrak{p}})$. Ist $k(\mathfrak{p})$ der zu $\mathfrak{p}$ gehörende Restekörper, dann ist $\tilde{\mathfrak{p}} \in \operatorname{spec}(k(\mathfrak{p}) \otimes A)$. Ist $\rho: \operatorname{spec}(k(\mathfrak{p}) \otimes A) \longrightarrow \operatorname{spec}(R \otimes A)$ die Faser über $\mathfrak{p}$, dann ist $\rho^{*} R\{\tau\}$ der $k(\mathfrak{p}) \otimes A$-Modul $k(\mathfrak{p})\{\tau\}$. Dieser ist nach 3.3.2 lokal frei von Rang $d$. Dann ist $\operatorname{dim}_{k(\tilde{\mathfrak{p}})} k(\tilde{\mathfrak{p}}) \otimes_{R \otimes A} R\{\tau\}=d$. Da $\tilde{\mathfrak{p}}$ beliebig gewählt ist, sind die Voraussetzungen von Satz 2.7 erfüllt.

\subsection{4 $R$ ist ein noetherscher Ring}

Dieser Fall wird mit Hilfe von Theorem 2.9 auf den Fall 3.3.3 zurückgeführt. Sei $R$ ein beliebiger Ring und sei $\mathfrak{n}$ sein Nilradikal. Dann ist auch $\mathfrak{n} \otimes A$ ein nilpotentes Ideal in $R \otimes A$. Es ist $R\{\tau\} /(\mathfrak{n} \otimes A) R\{\tau\} \cong(R / \mathfrak{n})\{\tau\}$ und $R \otimes A /(\mathfrak{n} \otimes A) \cong R / \mathfrak{n} \otimes A$. Da $R\{\tau\}$ ein freier $R$-Modul ist, gilt außerdem

$$
(\mathfrak{n} \otimes A) \otimes_{R \otimes A} R\{\tau\} \cong \mathfrak{n} \otimes_{R} R\{\tau\} \cong \mathfrak{n} R\{\tau\} .
$$

Für den reduzierter Ring $R / \mathfrak{n}$ ist nach 3.3.3 die Voraussetzung des Theorems 2.9 erfüllt. Der Modul $R\{\tau\}$ ist folglich ein flacher Modul, und da er endlich erzeugt ist, auch projektiv.

\subsection{5 $R$ ist ein beliebiger, kommutativer Ring}

Da der Dedekindring $A$ eine endlich erzeugte $\mathbb{F}_{q}$-Algebra ist, gibt es eine endlich erzeugte $\mathbb{F}_{q^{-}}$Unteralgebra $R^{\prime}$ von $R$, so dass die Bilder von $A$ bereits in $R^{\prime}\{\tau\}$ liegen. Nach 3.3.4 ist $R^{\prime}\{\tau\}$ ein lokal freier $R^{\prime} \otimes A$-Modul von Rang $d$. Die Behauptung folgt nun durch Skalarerweiterung. 


\subsection{Konstruktion der Faser in $\infty$}

Sei $(R, e)$ ein Drinfeld-Modul in Standardform über dem affinen Schema $S=$ $\operatorname{spec} R$ von Rang $d$.

Im Folgenden soll die Konstruktion eines Vektorbündels aus Abschnitt 2.8 angewandt werden. Nach Satz 3.8 ist $R\{\tau\}$ ein projektiver $R \otimes A$-Modul von Rang $d$. Gesucht ist ein projektiver $R \otimes \mathcal{O}_{X, \infty}$-Modul $\mathscr{E}_{\infty}$ von Rang $d$ und ein Isomorphismus

$$
\mathscr{E}_{\infty} \otimes_{\mathcal{O}_{X, \infty}} F \stackrel{\sim}{\longrightarrow} R\{\tau\} \otimes_{A} F .
$$

Mit Hilfe dieses Isomorphismus lässt sich $\mathscr{E}_{\infty}$ als Teilmenge von $R\{\tau\} \otimes_{A} F$ auffassen.

Durch Bildung des Hauptnenners ist jedes Element in $R\{\tau\} \otimes_{A} F$ von der Form $F(\tau) \otimes x$ mit $F(\tau) \in R\{\tau\}$ und $x \in F$. Man definiert eine Fortsetzung der natürlichen Gradabbildung auf $R\{\tau\}$ durch

$$
\begin{aligned}
\operatorname{deg}_{\tau}: R\{\tau\} \otimes_{A} F & \longrightarrow \mathbb{Z} \cup\{-\infty\} \\
F(\tau) \otimes x & \longmapsto \operatorname{deg}_{\tau} F(\tau)-d \operatorname{deg}(\infty) \infty(x) .
\end{aligned}
$$

Dies ist wohldefiniert, denn für alle $a \in A$ gilt

$$
\operatorname{deg}_{\tau} F(\tau) e(a)=\operatorname{deg}_{\tau} F(\tau)+\operatorname{deg}_{\tau} e(a),
$$

da der Leitkoeffizient von $e(a)$ eine Einheit in $R$ ist. Es gilt die starke Dreiecksungleichung

$$
\operatorname{deg}_{\tau}(\alpha+\beta) \leq \max \left(\operatorname{deg}_{\tau} \alpha, \operatorname{deg}_{\tau} \beta\right)
$$

und genau dann ist $F(\tau) \otimes x=0$ falls $F(\tau)=0 \vee x=0$ gilt.

Definition 3.9 ([BS97])

Für alle $i \in \mathbb{Z}$ sei $\mathscr{E}_{i, \infty}:=\left\{\alpha \in R\{\tau\} \otimes_{A} F \mid \operatorname{deg}_{\tau} \alpha \leq i\right\}$.

Sei $r \otimes x \in R \otimes \mathcal{O}_{X, \infty}$ (d.h. $\left.\infty(x) \geq 0\right)$ und $F(\tau) \otimes y \in \mathscr{E}_{i, \infty}$. Dann ist

$$
\begin{aligned}
\operatorname{deg}_{\tau}((r \otimes x) F(\tau) \otimes y) & =\operatorname{deg}_{\tau}(r F(\tau) \otimes x y) \\
& \leq \operatorname{deg}_{\tau}(F(\tau) \otimes y)-d \operatorname{deg}(\infty) \infty(x) \\
& \leq \operatorname{deg}_{\tau}(F(\tau) \otimes y) \leq i
\end{aligned}
$$

Insbesondere ist $\mathscr{E}_{i, \infty}$ ein $R \otimes \mathcal{O}_{X, \infty}$-Modul für alle $i \in \mathbb{Z}$.

\subsection{Projektivität des Moduls $\mathscr{E}_{i, \infty}$}

Satz 3.10

Für jedes $i \in \mathbb{Z}$ ist $\mathscr{E}_{i, \infty}$ ein endlich erzeugter, projektiver $R \otimes \mathcal{O}_{X, \infty}$-Modul von Rang $d$. 
Wir zeigen zunächst, dass $\mathscr{E}_{i, \infty}$ endlich erzeugt ist. Dann zeigen wir Satz 3.10 sukzessive für die folgenden vier Fälle:

3.5.1 $R=L$ ist ein Körper

3.5.2 $R$ ist ein reduzierter, noetherscher Ring.

3.5.3 $R$ ist ein noetherscher Ring.

3.5.4 $R$ ist ein beliebiger, kommutativer Ring.

\section{Lemma 3.11}

Sei $a \in A$ mit $k:=\operatorname{deg}_{\tau} e(a)>0$ und sei $\pi_{\infty} \in F$ mit $\infty\left(\pi_{\infty}\right)=1$. Dann ist der $R \otimes \mathcal{O}_{X, \infty}$-Modul $\mathscr{E}_{i, \infty}$ erzeugt von den Elementen

$$
\left\{\begin{array}{c|c}
\tau^{r} \otimes \pi_{\infty}^{s} & \begin{array}{c}
0 \leq r<k \\
\frac{-i}{d \operatorname{deg}(\infty)} \leq s \leq \frac{-i}{d \operatorname{deg}(\infty)}+\frac{k-1}{d \operatorname{deg}(\infty)}+1 \\
r-d \operatorname{deg}(\infty) s \leq i
\end{array}
\end{array}\right\}
$$

Insbesondere sind die $R \otimes \mathcal{O}_{X, \infty}-$ Moduln $\mathscr{E}_{i, \infty}$ endlich erzeugt für alle $i \in \mathbb{Z}$.

Beweis Die Nummerierung bezieht sich auf die zu zeigenden Ungleichungen.

1) Sei $F(\tau) \otimes x \in \mathscr{E}_{i, \infty}$. Ist $\operatorname{deg}_{\tau} F(\tau) \geq k$, dann liefert Division mit Rest $F(\tau)=H(\tau) e(a)+R(\tau) \operatorname{mit} \operatorname{deg}_{\tau} H(\tau) e(a)=\operatorname{deg}_{\tau} F(\tau)$ und $\operatorname{deg}_{\tau} R(\tau)<$ $k$. Es folgt

$$
\begin{aligned}
F(\tau) \otimes x & =H(\tau) e(a) \otimes x+R(\tau) \otimes x \\
& =H(\tau) \otimes a x+R(\tau) \otimes x .
\end{aligned}
$$

Beide Summanden liegen in $\mathscr{E}_{i, \infty}$. Nach Voraussetzung ist $\operatorname{deg}_{\tau} R(\tau)<$ $k . \mathrm{Da} \operatorname{deg}_{\tau} H(\tau)<\operatorname{deg}_{\tau} F(\tau)$ ist, können wir Induktion nach $\operatorname{deg}_{\tau} F(\tau)$ anwenden. Der $R \otimes \mathcal{O}_{X, \infty}$-Modul $\mathscr{E}_{i, \infty}$ wird also von Elementen der Form $\tau^{r} \otimes x$ mit $0 \leq r<k$ erzeugt.

2) Jedes $x \in F$ lässt sich schreiben als $x=\varepsilon \pi_{\infty}^{s}$ mit einem $\varepsilon \in \mathcal{O}_{X, \infty}^{\star}$ und $s=\infty(x)$. Das Element $F(\tau) \otimes x \in \mathscr{E}_{i, \infty}$ liegt folglich im Erzeugnis von $\tau^{r} \otimes \pi_{\infty}^{s}$ für $r-d \operatorname{deg}(\infty) s \leq i$.

3) Aus $0 \leq r<k$ folgt $s \geq-\frac{i}{d \operatorname{deg}(\infty)}$.

4) Ist $r-d \operatorname{deg}(\infty)(s-1) \leq i$, dann gilt

$$
\tau^{r} \otimes \pi_{\infty}^{s}=\left(1 \otimes \pi_{\infty}\right) \tau^{r} \otimes \pi_{\infty}^{s-1} \operatorname{mit} \tau^{r} \otimes \pi_{\infty}^{s-1} \in \mathscr{E}_{i, \infty} .
$$

Das heißt, $\tau^{r} \otimes \pi_{\infty}^{s}$ liegt im Erzeugnis von $\tau^{r} \otimes \pi_{\infty}^{s-1}$. Da $r<k$ ist, gilt dies insbesondere für $s \geq \frac{-i}{d \operatorname{deg}(\infty)}+\frac{k-1}{d \operatorname{deg}(\infty)}+1$. 


\section{Lemma 3.12}

Für alle $i \in \mathbb{Z}$ ist der $R$-Modul $\mathscr{E}_{i, \infty} / \mathscr{E}_{i-1, \infty}$ frei von Rang 1 .

Sei $\tau^{r} \otimes \pi_{\infty}^{s} \in L\{\tau\} \otimes_{A} F$ mit $r-d \operatorname{deg}(\infty) s=i$, also $\operatorname{deg}_{\tau} \tau^{r} \otimes \pi_{\infty}^{s}=i$. Dann ist $\tau^{r} \otimes \pi_{\infty}^{s}$ ein Basiselement von $\mathscr{E}_{i, \infty} / \mathscr{E}_{i-1, \infty}$ als $R$-Modul.

Beweis Man zeigt zunächst, dass $\tau^{r} \otimes \pi_{\infty}^{s}$ ein erzeugendes Element des Quotientenmoduls ist. Sei $F(\tau) \otimes x \in \mathscr{E}_{i, \infty} \backslash \mathscr{E}_{i-1, \infty}$ mit $F(\tau)=\sum_{t=0}^{n} \rho_{n} \tau^{n}$ und $\rho_{n} \neq 0$. Es gilt $F(\tau) \otimes x \equiv \rho_{n} \tau^{n} \otimes x \bmod \mathscr{E}_{i-1, \infty}$. Sei $\lambda \in F \operatorname{mit} \lambda \pi_{\infty}^{s}=x$. Es gibt $a, b \in A$ mit $\lambda=\frac{a}{b}$. Sei

$$
y:=\frac{\pi_{\infty}^{s}}{b}=\frac{x}{a} .
$$

Es folgt

$$
\begin{aligned}
\tau^{r} \otimes \pi_{\infty}^{s} & =\tau^{r} \otimes \frac{b}{b} \pi_{\infty}^{s}=\tau^{r} e(b) \otimes y \\
& \equiv \rho^{\prime} \tau^{r+\operatorname{deg}_{\tau}(e(b))} \otimes y \quad \bmod \mathscr{E}_{i-1, \infty} \\
\rho_{n} \tau^{n} \otimes x & =\rho_{n} \tau^{n} \otimes \frac{a}{a} x=\rho_{n} \tau^{n} e(a) \otimes y \\
& \equiv \rho^{\prime \prime} \tau^{n+\operatorname{deg}_{\tau}(e(a))} \otimes y \quad \bmod \mathscr{E}_{i-1, \infty}
\end{aligned}
$$

mit $\rho^{\prime}, \rho^{\prime \prime} \in R$. Nach Voraussetzung ist $\rho^{\prime}$ sogar eine Einheit. Weiterhin gilt

$$
\begin{gathered}
r+\operatorname{deg}_{\tau}(e(b))-d \operatorname{deg}(\infty) \infty(y)=i \\
n+\operatorname{deg}_{\tau}(e(a))-d \operatorname{deg}(\infty) \infty(y)=i
\end{gathered}
$$

also

$$
m:=r+\operatorname{deg}_{\tau}(e(b))=n+\operatorname{deg}_{\tau}(e(a)) .
$$

Da $\rho^{\prime}$ eine Einheit ist, gibt es $\rho \in R$ mit $\rho \rho^{\prime}=\rho^{\prime \prime}$. Dann gilt

$$
\rho \rho^{\prime} \tau^{m} \otimes y=\rho^{\prime \prime} \tau^{m} \otimes y
$$

und es folgt

$$
\rho\left(\tau^{r} \otimes \pi_{\infty}^{s}\right) \equiv F(\tau) \otimes x \quad \bmod \mathscr{E}_{i-1, \infty}
$$

Ist $\rho\left(\tau^{r} \otimes \pi_{\infty}^{s}\right)=0$, dann ist $\rho=0$, da $R$ torsionsfrei operiert. Es folgt die Behauptung.

\section{Lemma 3.13}

Für alle $\mathfrak{p} \nsupseteq R \otimes \mathfrak{m}_{X, \infty}$ und alle $i \in \mathbb{Z}$ gilt

$$
\left(\mathscr{E}_{i, \infty}\right)_{\mathfrak{p}}=\left(\mathscr{E}_{i+1, \infty}\right)_{\mathfrak{p}}=\left(R\{\tau\} \otimes_{A} F\right)_{\mathfrak{p}}
$$


Beweis Sei $\mathfrak{p} \in \operatorname{spec}\left(R \otimes \mathcal{O}_{X, \infty}\right)$ mit $\mathfrak{p} \nsupseteq R \otimes \mathfrak{m}_{X, \infty}$. Dann ist $1 \otimes \pi_{\infty} \notin \mathfrak{p}$, und $1 \otimes \pi_{\infty}$ ist eine Einheit in $\left(R \otimes \mathcal{O}_{X, \infty}\right)_{\mathfrak{p}}$. Aus $\mathscr{E}_{i, \infty} \subseteq \mathscr{E}_{i+1, \infty}$ folgt $\left(\mathscr{E}_{i, \infty}\right)_{\mathfrak{p}} \subseteq$ $\left(\mathscr{E}_{i+1, \infty}\right)_{\mathfrak{p}}$. Sei $\beta \in \mathscr{E}_{i+1, \infty}$. Dann ist $\left(1 \otimes \pi_{\infty}\right) \beta \in \mathscr{E}_{i, \infty}$ und es gilt

$$
\beta=\left(1 \otimes \pi_{\infty}\right)^{-1}\left(1 \otimes \pi_{\infty}\right) \beta \in\left(\mathscr{E}_{i, \infty}\right)_{\mathfrak{p}} .
$$

Es folgt $\left(\mathscr{E}_{i+1, \infty}\right)_{\mathfrak{p}} \subseteq\left(\mathscr{E}_{i, \infty}\right)_{\mathfrak{p}}$. Aus $\bigcup_{i \in \mathbb{Z}} \mathscr{E}_{i, \infty}=R\{\tau\} \otimes_{A} F$ folgt schließlich

$$
\bigcup_{i \in \mathbb{Z}}\left(\mathscr{E}_{i, \infty}\right)_{\mathfrak{p}}=\left(\mathscr{E}_{i, \infty}\right)_{\mathfrak{p}}=\left(R\{\tau\} \otimes_{A} F\right)_{\mathfrak{p}}
$$

\subsection{1 $\mathrm{R}=\mathrm{L}$ ist ein Körper}

Sei $P \in X, P \neq \infty$ und sei $B:=\Gamma\left(X \backslash\{P\}, \mathcal{O}_{X}\right)$. Die Ringe $L \otimes \mathcal{O}_{X, \infty}$ und $L \otimes F$ sind Lokalisierungen des Dedekindringes $L \otimes B$ und deshalb Dedekindringe (vgl. Satz 2.21, Folgerung 2.26 und Bemerkung 2.4).

Nach Satz 3.8 ist $L\{\tau\}$ ein projektiver $L \otimes A$-Modul. Dann ist auch $L\{\tau\} \otimes_{A}$ $F$ ein projektiver, insbesondere torsionsfreier $L \otimes F$-Modul. Der Unterring $L \otimes \mathcal{O}_{X, \infty}$ operiert also torsionsfrei auf $L\{\tau\} \otimes_{A} F$ und damit auch auf $\mathscr{E}_{i, \infty}$ für alle $i \in \mathbb{Z}$.

Als endlich erzeugte, torsionsfreie Moduln über dem Dedekindring $L \otimes \mathcal{O}_{X, \infty}$ sind die Moduln $\mathscr{E}_{i, \infty}$ projektiv (vgl. 2.5).

$\mathrm{Da}(L \otimes F)_{(0)}=\left(L \otimes \mathcal{O}_{X, \infty}\right)_{(0)}$ und $(0) \nsupseteq R \otimes \mathfrak{m}_{X, \infty}$ gilt, ist nach Lemma $3.13\left(\mathscr{E}_{i, \infty}\right)_{(0)}=\left(L\{\tau\} \otimes_{A} F\right)_{(0)}$. Der $(L \otimes F)_{(0)}$-Modul $\left(L\{\tau\} \otimes_{A} F\right)_{(0)}$ hat als Lokalisierung des $L \otimes F$-Moduls $L\{\tau\} \otimes_{A} F$ den Rang $d$.

\section{Lemma 3.14}

1) Sei $R$ ein beliebiger Ring über $\mathbb{F}_{q}$, sei $\mathfrak{a}$ ein Ideal und sei $\bar{R}:=R / \mathfrak{a}$. Ist $(R, e)$ ein Drinfeld-Modul in Standardform von Rang d, dann ist auch $(\bar{R}, \bar{e})$ ein Drinfeld-Modul von Rang d in Standardform.

Sei

$$
\overline{\mathscr{E}}_{i, \infty}:=\left\{\bar{\alpha} \in \bar{R}\{\tau\} \otimes_{A} F \mid \operatorname{deg}_{\tau} \bar{\alpha} \leq i\right\},
$$

dann ist die Abbildung

$$
\begin{gathered}
\mathscr{E}_{i, \infty} /\left(\mathfrak{a} \otimes \mathcal{O}_{X, \infty}\right) \mathscr{E}_{i, \infty} \longrightarrow \overline{\mathscr{E}}_{i, \infty} \\
F(\tau) \otimes x \bmod \left(\mathfrak{a} \otimes \mathcal{O}_{X, \infty}\right) \mathscr{E}_{i, \infty} \longmapsto \bar{F}(\tau) \otimes x
\end{gathered}
$$

ein Isomorphismus von $\bar{R} \otimes \mathcal{O}_{X, \infty}-$ Moduln.

2) Sei $S$ eine multiplikativ abgeschlossene Teilmenge von $R$.

Sei $S \otimes 1:=\left\{s \otimes 1 \in R \otimes \mathcal{O}_{X, \infty} \mid s \in S\right\}$. Dann ist auch $S \otimes 1$ multiplikativ abgeschlossen und es gilt

$$
(S \otimes 1)^{-1}\left(R \otimes \mathcal{O}_{X, \infty}\right)=S^{-1} R \otimes \mathcal{O}_{X, \infty}
$$


Ist $(R, e)$ ein Drinfeld-Modul von Rang d in Standardform, dann ist auch die kanonische Fortsetzung auf $\left(S^{-1} R, e\right)$ ein Drinfeld-Modul von Rangd in Standardform. Sei

$$
\tilde{\mathscr{E}}_{i, \infty}:=\left\{\alpha \in S^{-1} R\{\tau\} \otimes F \mid \operatorname{deg}_{\tau} \alpha \leq i\right\},
$$

dann ist die Abbildung

$$
\begin{gathered}
(S \otimes 1)^{-1} \mathscr{E}_{i, \infty} \longrightarrow \tilde{\mathscr{E}}_{i, \infty} \\
(s \otimes 1)^{-1}(F(\tau) \otimes x) \longmapsto s^{-1} F(\tau) \otimes x
\end{gathered}
$$

ein Isomorphismus von $S^{-1} R \otimes \mathcal{O}_{X, \infty}-$ Moduln.

\section{Beweis}

1) Die Abbildung ist wohldefiniert, da $\operatorname{deg}_{\tau} \bar{F}(\tau) \leq \operatorname{deg}_{\tau} F(\tau)$ gilt. Mit $\tau^{r} \otimes$ $\pi_{\infty}^{s}$ für $r-d \operatorname{deg}(\infty) s \leq i$ ist auch $\bar{\tau}^{r} \otimes \pi_{\infty}^{s}$ für $r-d \operatorname{deg}(\infty) s \leq i$ ein Erzeugendensystem von $\overline{\mathscr{E}}_{i, \infty}$, es folgt die Surjektivität der Abbildung.

Sei $\bar{F}(\tau) \otimes x=0$. Aus $x \neq 0$ folgt, dass $\bar{F}(\tau)=0$ ist, das heißt $F(\tau) \in$ $\mathfrak{a}\{\tau\}$. Es folgt die Injektivität der Abbildung.

2) Der Beweis von Teil 2) ist analog zu Teil 1).

\subsection{2 $\mathrm{R}$ ist ein reduzierter, noetherscher $\mathrm{Ring}$}

Ist $\tilde{y} \in \operatorname{spec}\left(R \otimes \mathcal{O}_{X, \infty}\right)$, dann gibt es ein $y \in \operatorname{spec} R$, so dass $\tilde{y}$ in der Faser über $y$ bezüglich der Projektion $\pi: \operatorname{spec}\left(R \otimes \mathcal{O}_{X, \infty}\right) \longrightarrow \operatorname{spec} R$ liegt. Ist $k(y)$ der zu $y$ gehörende Restekörper, dann ist $\tilde{y} \in \operatorname{spec}\left(k(y) \otimes \mathcal{O}_{X, \infty}\right)$. Ist $\rho: \operatorname{spec}\left(k(y) \otimes \mathcal{O}_{X, \infty}\right) \longrightarrow \operatorname{spec}\left(R \otimes \mathcal{O}_{X, \infty}\right)$ die Faser über $y$, dann ist $\rho^{*} \mathscr{E}_{i, \infty}$ $\operatorname{der} k(y) \otimes \mathcal{O}_{X, \infty}$-Modul $\tilde{\bar{E}}_{i, \infty}$ für ein geeignetes Ideal $\mathfrak{p}$ in $R$ und eine geeignete multiplikativ abgeschlossene Teilmenge $S$ nach Lemma 3.14,1) und 2). Dieser ist nach 3.5.1 lokal frei von Rang $d$. Dann ist $\operatorname{dim}_{k(\tilde{y})} k(\tilde{y}) \otimes_{R \otimes \mathcal{O}_{X, \infty}} \mathscr{E}_{i, \infty}=d$. Da $\tilde{y}$ beliebig gewählt werden kann, sind die Voraussetzungen von Satz 2.7 erfüllt.

\subsection{3 $\mathrm{R}$ ist ein noetherscher Ring}

Wie im Beweis zu 3.8, Abschnitt 3.3.4, ist die Injektivität der kanonischen Abbildung $\mathfrak{n} \otimes_{R} \mathscr{E}_{i, \infty} \longrightarrow \mathfrak{n} \mathscr{E}_{i, \infty}$ für das Nilradikal $\mathfrak{n}$ von $R$ zu zeigen. Man zeigt sogar, dass $\mathscr{E}_{i, \infty}$ ein flacher $R$-Modul für alle $i \in \mathbb{Z}$ ist.

Da $R\{\tau\}$ ein projektiver $R \otimes A$-Modul ist, folgt, dass auch $R\{\tau\} \otimes F$ ein projektiver $R \otimes F$-Modul ist. Andererseits ist auch $R \otimes F$ ein projektiver 
(sogar freier) $R$-Modul. Es folgt, dass $R\{\tau\} \otimes F$ ein projektiver und damit flacher $R$-Modul ist.

Sei

$$
0 \longrightarrow \mathscr{E}_{i, \infty} \longrightarrow \mathscr{E}_{i+1, \infty} \longrightarrow \mathscr{E}_{i+1, \infty} / \mathscr{E}_{i, \infty} \longrightarrow 0
$$

die kanonische exakte Sequenz und sei $\mathfrak{a}$ ein Ideal in $R$. Tensorieren der Sequenz mit $\mathfrak{a}$ liefert

$$
\begin{array}{r}
\longrightarrow \operatorname{Tor}^{1}\left(\mathfrak{a}, \mathscr{E}_{i+1, \infty} / \mathscr{E}_{i, \infty}\right) \longrightarrow \mathfrak{a} \otimes_{R} \mathscr{E}_{i, \infty} \longrightarrow \\
\mathfrak{a} \otimes_{R} \mathscr{E}_{i+1, \infty} \longrightarrow \mathfrak{a} \otimes_{R} \mathscr{E}_{i+1, \infty} / \mathscr{E}_{i, \infty} \longrightarrow 0
\end{array}
$$

In 3.12 wurde gezeigt, dass $\mathscr{E}_{i+1, \infty} / \mathscr{E}_{i, \infty}$ ein projektiver $R$-Modul ist. Die Torsion ist also gleich Null. Also ist die Abbildung

$$
\mathfrak{a} \otimes_{R} \mathscr{E}_{i, \infty} \longrightarrow \mathfrak{a} \otimes_{R} \mathscr{E}_{i+1, \infty}
$$

injektiv.

Ist $K_{i}:=\operatorname{Ker}\left(\mathfrak{a} \otimes_{R} \mathscr{E}_{i, \infty} \longrightarrow \mathfrak{a} \mathscr{E}_{i, \infty}\right)$, dann folgt aus obigem, dass die natürliche Abbildung $K_{i} \longrightarrow K_{i+1}$ ebenfalls injektiv ist.

Ist

$$
0 \longrightarrow K_{i} \longrightarrow \mathfrak{a} \otimes_{R} \mathscr{E}_{i, \infty} \longrightarrow \mathfrak{a} \mathscr{E}_{i, \infty} \longrightarrow 0
$$

die kanonische exakte Sequenz, dann liefert die Anwendung des exakten Funktors $\underline{\lim }$ die exakte Sequenz

$$
0 \longrightarrow \lim _{\longrightarrow} K_{i} \longrightarrow \underline{\lim }\left(\mathfrak{a} \otimes_{R} \mathscr{E}_{i, \infty}\right) \longrightarrow \lim _{\mathfrak{a}} \mathfrak{\mathscr { E } _ { i , \infty }} \longrightarrow 0
$$

Außerdem gilt $\lim \left(\mathfrak{a} \otimes_{R} \mathscr{E}_{i, \infty}\right)=\mathfrak{a} \otimes_{R} \lim \mathscr{E}_{i, \infty}=\mathfrak{a} \otimes_{R}(R\{\tau\} \otimes F)$. Da $R\{\tau\} \otimes F$ projektiver $R$-Modul ist, ist $\lim _{i} K_{i}=0$. Die im Limes auftretenden Abbildungen sind alle injektiv, daher ist $K_{i}=0$ für alle $i \in \mathbb{Z}$. Nach [Mat86] §7, Theorem 7.7, sind die $R$-Moduln $\mathscr{E}_{i, \infty}$ flach.

\subsection{4 $R$ ist ein beliebiger Ring}

Nach 3.11 ist $\mathscr{E}_{i, \infty}$ ein endlich erzeugter $R \otimes \mathcal{O}_{X, \infty}$-Modul. Seien $m_{1}, \ldots, m_{r} \in$ $\mathscr{E}_{i, \infty} \subseteq R\{\tau\} \otimes_{A} F$ Erzeuger. Dann gibt es eine endlich erzeugte $\mathbb{F}_{q}$-Unteralgebra $R^{\prime}$ von $R$, so dass $m_{1}, \ldots, m_{r}$ bereits in $R^{\prime}\{\tau\} \otimes_{A} F$ liegen. Sei $\mathscr{E}_{i, \infty}^{\prime}$ der zum Ring $R^{\prime}$ gehörende Untermodul von $R^{\prime}\{\tau\} \otimes_{A} F$. Die kanonische Abbildung

$$
R \otimes_{R^{\prime}} \mathscr{E}_{i, \infty}^{\prime} \longrightarrow \mathscr{E}_{i, \infty}
$$

ist in diesem Fall ein Isomorphismus. Da $\mathscr{E}_{i, \infty}$ eine Skalarerweiterung des Moduls $\mathscr{E}_{i, \infty}^{\prime}$ ist, folgt die Behauptung und Satz 3.10 ist bewiesen. 


\subsection{Konstruktion des Vektorbündels}

Mit Hilfe der Ergebnisse aus den Abschnitten 3.3 und 3.5 können wir für alle $i \in \mathbb{Z}$ ein $\mathcal{O}_{X \times S^{-}}$Vektorbündel konstruieren. Außerdem erhalten wir kanonische Abbildungen

$$
s_{i}: \mathscr{E}_{i} \longrightarrow \mathscr{E}_{i+1}
$$

und

$$
t_{i}:{ }^{\tau} \mathscr{E}_{i} \longrightarrow \mathscr{E}_{i+1}
$$

für alle $i \in \mathbb{Z}$.

Lemma 3.15

Sei $\pi_{\infty} \in F$ mit $\infty\left(\pi_{\infty}\right)=1$. Dann ist die Abbildung

$$
\begin{gathered}
\mathscr{E}_{i, \infty} \longrightarrow \mathscr{E}_{i+d \operatorname{deg}(\infty), \infty} \\
\alpha \longmapsto \alpha \pi_{\infty}^{-1}
\end{gathered}
$$

ein Isomorphismus von $R \otimes \mathcal{O}_{X, \infty}$-Moduln. Die Umkehrabbildung ist

$$
\begin{aligned}
\mathscr{E}_{i+d \operatorname{deg}(\infty), \infty} \longrightarrow \mathscr{E}_{i, \infty} \\
\alpha \longmapsto \alpha \pi_{\infty} .
\end{aligned}
$$

Beweis Die Behauptung folgt aus der Gültigkeit der Gleichung

$$
\operatorname{deg}_{\tau}(\alpha x)=\operatorname{deg}_{\tau} \alpha-d \operatorname{deg}(\infty) \infty(x)
$$

für alle $\alpha \in R\{\tau\} \otimes_{A} F$ und $x \in F$.

Sei $F(\tau) \otimes x \in R\{\tau\} \otimes_{A} F$ von Grad $i$. Dann ist $\operatorname{deg}_{\tau}(\tau F(\tau) \otimes x) \leq i+1$. Dies induziert für alle $i \in \mathbb{Z}$ eine Abbildung

$$
\begin{gathered}
\mathscr{E}_{i, \infty} \longrightarrow \mathscr{E}_{i+1, \infty} \\
F(\tau) \otimes x \longmapsto \tau F(\tau) \otimes x .
\end{gathered}
$$

Sie ist linear in $\mathcal{O}_{X, \infty}$ und $q$-linear in $R$. Nach Satz 2.78 faktorisiert sie über $\tau_{\mathscr{E}_{i, \infty}}$. Es gilt:

\section{Lemma 3.16}

Sei $\mathfrak{p}$ Primideal in $R \otimes \mathcal{O}_{X, \infty}$ mit $\mathfrak{p} \supseteq R \otimes \mathfrak{m}_{X, \infty}$. Dann ist

$$
\begin{aligned}
\left({ }^{\tau} \mathscr{E}_{i, \infty}\right)_{\mathfrak{p}} & \longrightarrow\left(\mathscr{E}_{i+1, \infty}\right)_{\mathfrak{p}} \\
1 \otimes \alpha & \longmapsto \tau \alpha
\end{aligned}
$$

ein Isomorphismus von $\left(R \otimes \mathcal{O}_{X, \infty}\right)_{\mathfrak{p}}-\operatorname{Moduln}$. 
Beweis Der Modul $\left(\mathscr{E}_{i, \infty}\right)_{\mathfrak{p}}$ ist ein endlich erzeugter $\left(R \otimes \mathcal{O}_{X, \infty}\right)_{\mathfrak{p}^{-}}$-Modul und das Ideal $R \otimes \mathfrak{m}_{X, \infty} \subseteq \operatorname{Rad}\left(\left(R \otimes \mathcal{O}_{X, \infty}\right)_{\mathfrak{p}}\right)=\mathfrak{p}$. Nach Lemma 3.11 wird $\mathscr{E}_{i, \infty}$ erzeugt von $\tau^{r} \otimes \pi_{\infty}^{s}$ mit $r, s$ wie in 3.11. Sei $M$ der von $\tau^{r+1} \otimes \pi_{\infty}^{s}$, erzeugte Untermodul in $\left(\mathscr{E}_{i+1, \infty}\right)_{\mathfrak{p}}$. Nach dem Nakayama-Lemma ([Mat86], Korollar zu Theorem 2.2) ist zu zeigen, dass

$$
\left(\mathscr{E}_{i+1, \infty}\right)_{\mathfrak{p}}=M+\left(R \otimes \mathfrak{m}_{X, \infty}\right)\left(\mathscr{E}_{i+1, \infty}\right)_{\mathfrak{p}}
$$

gilt. Da $\tau^{r} \otimes \pi_{\infty}^{s}$ ein Erzeugendensystem von $\left(\mathscr{E}_{i+1, \infty}\right)_{\mathfrak{p}}$ bildet und die Elemente $\tau^{r} \otimes \pi_{\infty}^{s}$ für $r>0$ bereits in $M$ sind, reicht es zu zeigen, dass $1 \otimes \pi_{\infty}^{s} \in$ $\left(R \otimes \mathfrak{m}_{X, \infty}\right)\left(\mathscr{E}_{i+1, \infty}\right)_{\mathfrak{p}}$ gilt. Man betrachtet nun die folgenden beiden Fälle:

Fall I Ist $-d \operatorname{deg} \infty(s-1) \leq i+1$. Dann ist

$$
1 \otimes \pi_{\infty}^{s}=\left(1 \otimes \pi_{\infty}\right) 1 \otimes \pi_{\infty}^{s-1} \in\left(R \otimes \mathfrak{m}_{X, \infty}\right)\left(\mathscr{E}_{i+1, \infty}\right)_{\mathfrak{p}}
$$

Fall II Ist $-d \operatorname{deg} \infty(s-1)>i+1$. Dann ist

$$
\begin{aligned}
1 \otimes \pi_{\infty}^{s} & =1 \otimes \pi_{\infty}^{s} \frac{a}{a} \\
& =e(a) \otimes \frac{\pi_{\infty}^{s}}{a} \\
& =\sum_{n=0}^{k} r_{n} \tau^{n} \otimes \frac{\pi_{\infty}^{s}}{a} .
\end{aligned}
$$

Für $n>0$ liegen die Summanden bereits in $M$. Ist $r_{0}=0$, dann ist nichts mehr zu zeigen. Andernfalls ist $-\left(s-\frac{k}{d \operatorname{deg} \infty}\right) d \operatorname{deg}(\infty) \leq i+1$, insbesondere $-(s-1) d \operatorname{deg}(\infty) \leq i+1$ und man kann Fall I anwenden.

\section{Lemma 3.17}

Sei $\pi_{\infty} \in F$ mit $\infty\left(\pi_{\infty}\right)=1$. Dann ist die Abbildung

$$
\begin{aligned}
& \mathscr{E}_{i, \infty} \otimes_{\mathcal{O}_{X, \infty}} \pi_{\infty}^{-1} \mathcal{O}_{X, \infty} \longrightarrow \mathscr{E}_{i+d \operatorname{deg} \infty, \infty} \\
& \alpha \otimes \pi_{\infty}^{-1} x \longmapsto \alpha \pi_{\infty}^{-1} x
\end{aligned}
$$

ein Isomorphismus von $R \otimes \mathcal{O}_{X, \infty}-$ Moduln. Der Twist mit $\pi_{\infty} \mathcal{O}_{X, \infty}$ ergibt einen Isomorphismus mit $\mathscr{E}_{i-d \operatorname{deg} \infty, \infty}$.

Beweis Sei $\alpha \in \mathscr{E}_{i+d \operatorname{deg} \infty, \infty}$. Dann ist $\alpha \pi_{\infty} \in \mathscr{E}_{i, \infty}$ ein Urbild. Mit $\mathscr{E}_{i, \infty}$ ist auch $\mathscr{E}_{i, \infty} \otimes_{\mathcal{O}_{X, \infty}} \pi_{\infty}^{-1} \mathcal{O}_{X, \infty}$ projektiv von Rang $d$. Da surjektive Abbildungen projektiver Moduln von selbem Rang Isomorphismen sind, folgt die Behauptung. Die zweite Behauptung folgt analog. 


\section{Bemerkung 3.18}

Sei $(R, e)$ ein beliebiger Drinfeld-Modul über $A$. Nach Satz 3.6, 3), gibt es einen Drinfeld-Modul $(R, f)$ in Standardform und einen eindeutigen Isomorphismus $u:(R, e) \longrightarrow(R, f)$ von Drinfeld-Moduln mit $\partial u=1$.

Nach Lemma 3.7 induziert $u$ einen Isomorphismus der beiden $R \otimes A$ ModulStrukturen auf $R\{\tau\}$, also auch einen Isomorphismus

$$
\begin{aligned}
R\{\tau\} \otimes_{A, e} F & \stackrel{\sim}{\longrightarrow} R\{\tau\} \otimes_{A, f} F \\
F(\tau) \otimes x & \longmapsto F(\tau) u^{-1} \otimes x
\end{aligned}
$$

von $R \otimes F$-Moduln. Für den Drinfeld-Modul $(R, e)$ definiert man nun die $R \otimes \mathcal{O}_{X, \infty}$-Moduln $\mathscr{E}_{i, \infty} \subseteq R\{\tau\} \otimes_{A, e} F$ als Urbilder der bereits definierten Moduln $\mathscr{E}_{i, \infty} \subseteq R\{\tau\} \otimes_{A, f} F$. Insbesondere übertragen sich alle gezeigten Eigenschaften.

\section{Bemerkung 3.19}

Mit Hilfe von Satz 2.72, Satz 3.8 und Satz 3.10 können wir für alle $i \in \mathbb{Z}$ das $\mathcal{O}_{X \times S^{-}}$Vektorbündel $\left\{\mathscr{E}_{i}\right\}_{i \in \mathbb{Z}}$ von Rang $d$ konstruieren.

Für alle $i \in \mathbb{Z}$ wird durch die Inklusion der Moduln $\mathscr{E}_{i, \infty} \longrightarrow \mathscr{E}_{i+1, \infty}$ eine Abbildung

$$
s_{i}: \mathscr{E}_{i} \longrightarrow \mathscr{E}_{i+1}
$$

vermittelt. Die Linksmultiplikation mit $\tau$ definiert für alle $i \in \mathbb{Z}$ eine $\mathcal{O}_{S^{-}} q$ lineare Abbildung $\mathscr{E}_{i, \infty} \longleftrightarrow \mathscr{E}_{i+1, \infty}$. Mit Hilfe von Satz 2.78 erhalten wir also für alle $i \in \mathbb{Z}$ eine Abbildung

$$
t_{i}: \mathscr{E}_{i} \longrightarrow \mathscr{E}_{i+1}
$$

\section{Folgerung 3.20}

Sei $(R, e)$ ein Drinfeld-Modul in Standard-Form. Dann gilt für alle $i \in \mathbb{Z}$

$$
\mathscr{E}_{i}(X \times S)=\left\{F(\tau) \in R\{\tau\} \mid \operatorname{deg}_{\tau} F(\tau) \leq i\right\} .
$$

Beweis Nach Konstruktion ist das Klebedatum von $\mathscr{E}_{i, \infty}$ und $R\{\tau\}$ durch die kanonischen Abbildungen

$$
\mathscr{E}_{i, \infty} \longrightarrow R\{\tau\} \otimes_{A} F \longleftarrow R\{\tau\}
$$

gegeben. Aus der Definition von $\mathscr{E}_{i, \infty}$ folgt die Behauptung.

\section{Folgerung 3.21}

In der Situation von Lemma 3.16 gilt:

1) $H^{0}\left(X \times S, \mathscr{E}_{i}\right)=H^{0}\left(X \times s,\left(\mathscr{E}_{i}\right)_{s}\right)=0$ für $i<0$ und $s \in S$. 
2) $H^{1}\left(X \times S, \mathscr{E}_{i}\right)=H^{1}\left(X \times s,\left(\mathscr{E}_{i}\right)_{s}\right)=0$ für $i \geq-1$ und $s \in S$.

3) $\lim _{i \in \mathbb{Z}} H^{0}\left(X \times S, \mathscr{E}_{i}\right)=R\{\tau\}$.

\section{Beweis}

1) Folgt aus Folgerung 3.20.

2) Nach Lemma 3.13 hat $\mathscr{E}_{i} / \mathscr{E}_{i-1}$ nur Träger in $\operatorname{spec} \mathcal{O}_{X, \infty} \otimes R$ und es gilt nach Konstruktion

$$
\left.\left(\mathscr{E}_{i} / \mathscr{E}_{i-1}\right)\right|_{\operatorname{spec} \mathcal{O}_{X, \infty} \otimes R} \cong \mathscr{E}_{i, \infty} / \mathscr{E}_{i-1, \infty}
$$

Nach Lemma 3.12 ist für alle $i \geq 0$ das Element $\tau^{i} \in R\{\tau\}$ ein globaler Schnitt, der $\mathscr{E}_{i, \infty} / \mathscr{E}_{i-1, \infty}$ erzeugt. Insbesondere ist

$$
H^{0}\left(X \times S, \mathscr{E}_{i}\right) \longrightarrow H^{0}\left(X \times S, \mathscr{E}_{i} / \mathscr{E}_{i-1}\right)
$$

eine surjektive Abbildung und also $H^{1}\left(X \times S, \mathscr{E}_{i-1}\right)=0$ für alle $i \geq 0$.

3) Folgt aus Folgerung 3.20.

\subsection{Alternative Konstruktion}

Anstatt der expliziten Konstruktion der Halme im Punkt $\infty$ können wir die Vektorbündel $\mathscr{E}_{i}$ auch durch eine Proj-Konstruktion erhalten.

Sei $\mathcal{S}_{X}:=\bigoplus_{i=0}^{\infty} H^{0}\left(X, \mathcal{O}_{X}(i \infty)\right)$. Nach Satz 2.37 gilt Proj $\mathcal{S}_{X} \cong X$. Sei für alle $k \in \mathbb{Z}$

$$
M_{k}:=\left\{f(\tau) \in R\{\tau\} \mid \operatorname{deg}_{\tau} f(\tau) \leq k\right\}
$$

und sei für alle $i \in \mathbb{Z}$

$$
\mathcal{M}_{i}:=\bigoplus_{k=0}^{\infty} M_{k d \operatorname{deg}(\infty)+i}
$$

Für alle $i \in \mathbb{Z}$ ist $\mathcal{M}_{i}$ in natürlicher Weise ein graduierter $R \otimes \mathcal{S}_{X}$-Modul. Sei

$$
\mathscr{E}_{i}^{\prime}:=\mathcal{M}_{i}^{\sim}
$$

für alle $i \in \mathbb{Z}$ der durch die Proj-Konstruktion entstandene $\mathcal{O}_{X \times S^{-}}$Modul.

\section{Satz 3.22}

Für alle $i \in \mathbb{Z}$ sind die $\mathcal{O}_{X \times S}-$ Moduln $\mathscr{E}_{i}^{\prime}$ und $\mathscr{E}_{i}$ kanonisch isomorph. 
Beweis Sei $U:=X \backslash \infty$. Trivialerweise gilt

$$
\left.\mathscr{E}_{i}^{\prime}\right|_{U \times S}=\mathcal{M}_{i}\left[e^{-1}\right]_{(0)}=R\{\tau\}=\left.\mathscr{E}_{i}\right|_{U \times S}
$$

für alle $i \in \mathbb{Z}$. Sei $\mathfrak{p}_{\infty} \subseteq \mathcal{S}_{X}$ das zum Punkt $\infty$ gehörende homogene Primideal. Wir erhalten

$$
\begin{aligned}
\left.\mathscr{E}_{i}^{\prime}\right|_{\operatorname{spec} \mathcal{O}_{X, \infty} \times S} & \cong \mathcal{M}_{i\left(\mathfrak{p}_{\infty}\right)} \\
& \cong\left\{\frac{f(\tau)}{a} \mid \operatorname{deg}_{\tau} f(\tau) \leq \operatorname{deg}_{\tau}\left(e_{a}\right)+i, 0 \neq a \in A, f(\tau) \in R\{\tau\}\right\} \\
& \left.\cong \mathscr{E}_{i}\right|_{\operatorname{spec} \mathcal{O}_{X, \infty} \times S}
\end{aligned}
$$

für alle $i \in \mathbb{Z}$. Es folgt die Behauptung.

\subsection{Konstruktion über einem beliebigen Basisschema}

Im Folgenden wird die beschriebene Konstruktion auf den Fall eines beliebigen Basisschemas $S$ ausgedehnt. Sei $(E, e)$ ein Drinfeld-Modul von Rang $d$ über $S$ in Standardform. Sei

$$
\mathscr{E}_{U}:=\mathscr{H}_{o m_{\mathbb{F}_{q}}}\left(\mathbb{G}_{a / \mathscr{L}}, \mathbb{G}_{a / S}\right)
$$

Da $\mathcal{O}_{S} \subseteq \mathscr{E} n d_{\mathbb{F}_{q}}\left(\mathbb{G}_{a / S}\right)$ ist, operiert $\mathcal{O}_{S}$ in kanonischer Weise von rechts auf $\mathscr{E}_{U}$. Mittels der durch die Drinfeld-Modul-Struktur gegebenen Abbildung

$$
e: A \longrightarrow \mathscr{E} n d_{\mathbb{F}_{q}}\left(\mathbb{G}_{a / \mathscr{L}}\right)
$$

trägt $\mathscr{E}_{U}$ auch eine kanonische, von links operierende $A$-Modul-Struktur. Beide Operationen sind miteinander verträglich und machen $\mathscr{E}_{U} \mathrm{zu}$ einem $\mathcal{O}_{\text {spec } A \times S^{-M o d u l} \text {. }}$

Nach Satz 3.1 ist

$$
\mathscr{H}_{0 m_{\mathbb{F}_{q}}}\left(\mathbb{G}_{a / \mathscr{L}}, \mathbb{G}_{a / S}\right) \cong \bigoplus_{n \geq 0} \mathscr{L}^{-q^{n}}
$$

Wir definieren

$$
\mathcal{M}:=\bigoplus_{k=0}^{\infty}\left(\bigoplus_{n=0}^{k d \operatorname{deg} \infty} \mathscr{L}^{-q^{n}}\right)
$$

Seien

$$
\begin{aligned}
f: \mathscr{L} \longrightarrow \mathscr{L}^{-q^{n}} & \in \mathscr{E} n d_{\mathbb{F}_{q}}\left(\mathbb{G}_{a / \mathscr{L}}\right) \\
g: \mathcal{O}_{S} \longrightarrow \mathscr{L}^{-q^{m}} & \in \mathscr{H} o m_{\mathbb{F}_{q}}\left(\mathbb{G}_{a / \mathscr{L}}, \mathbb{G}_{a / S}\right)
\end{aligned}
$$


dann ist die Komposition der beiden Abbildungen

$$
g \circ f: \mathcal{O}_{S} \longrightarrow \mathscr{L}^{-q^{m+n}} \in \mathscr{H}_{o m_{\mathbb{F}_{q}}}\left(\mathbb{G}_{a / \mathscr{L}}, \mathbb{G}_{a / S}\right)
$$

wohldefiniert und in kanonischer Weise wird $\mathcal{M}$ zu einem graduierten $\mathcal{S}_{X} \otimes$ $\mathcal{O}_{S}$-Modul. Wir erhalten durch Anwendung des Proj-Funktors den quasikohärenten $\mathcal{O}_{X \times S^{-}}$Modul $\mathscr{E}$. Die Konstruktion der Garbe $\mathscr{E}$ stimmt lokal in $S$ mit der Konstruktion der Garbe $\mathscr{E}_{0}^{\prime}$ in Abschnitt 3.7 überein. Insbesondere ist Garbe $\mathscr{E}$ ein $\mathcal{O}_{X \times S^{-}}$Vektorbündel von Rang $d$.

Für alle $i \in \mathbb{Z}$ lassen sich mit Hilfe des Proj-Funktors, angewandt auf die $\mathcal{O}_{\text {spec } A \times S^{-}}$Moduln

$$
\mathcal{M}_{i}:=\bigoplus_{k=0}^{\infty}\left(\bigoplus_{n=0}^{i+k d \operatorname{deg} \infty} \mathscr{L}^{-q^{n}}\right),
$$

die $\mathcal{O}_{X \times S^{-}}$Moduln $\mathscr{E}_{i}$ gewinnen. Auch sie stimmen lokal in $S$ mit der Konstruktion in Abschnitt 3.7 überein. 


\section{Vektorbündel von allgemeinem Typ}

\subsection{Definition der Vektorbündel von allgemeinem Typ}

Definition 4.1 (Drinfeld, [Dri86])

Ein $\mathcal{O}_{X \times S^{-}}$Vektorbündel $\mathscr{E}$ heißt von allgemeinem Typ, falls für alle $i \in \mathbb{Z}$

$$
\begin{aligned}
H^{0}\left(X \times s, \mathscr{E}(i \infty)_{s}\right)=0 & \forall s \in S \\
\text { oder } & \\
H^{1}\left(X \times s, \mathscr{E}(i \infty)_{s}\right)=0 & \forall s \in S .
\end{aligned}
$$

gilt.

\section{Lemma 4.2}

1) Sei $\mathscr{E}$ ein $\mathcal{O}_{X \times S^{-V}}$ Vektorbündel von allgemeinem Typ. Dann ist für alle $k \in \mathbb{Z}$ auch $\mathscr{E}(k \infty)$ von allgemeinem Typ.

2) Sei $\mathscr{E}$ ein $\mathcal{O}_{X \times S^{-}}$Vektorbündel mit

$$
\begin{aligned}
& H^{0}\left(X \times s, \mathscr{E}_{s}\right)=0 \forall s \in S \\
& \text { und } \\
& H^{1}\left(X \times s, \mathscr{E}_{s}\right)=0 \quad \forall s \in S .
\end{aligned}
$$

dann ist $\mathscr{E}$ von allgemeinem Typ.

Beweis 1) ergibt sich direkt aus der Definition. Behauptung 2) ergibt sich aus der kanonischen Injektion

$$
H^{0}\left(X \times s, \mathscr{E}(i \infty)_{s}\right) \smile H^{0}\left(X \times s, \mathscr{E}((i+1) \infty)_{s}\right)
$$

und aus der Surjektion

$$
H^{1}\left(X \times s, \mathscr{E}(i \infty)_{s}\right) \longrightarrow H^{1}\left(X \times s, \mathscr{E}((i+1) \infty)_{s}\right)
$$

für alle $i \in \mathbb{Z}$ und alle $s \in S$.

\subsection{Drinfelds Theorem}

Theorem 4.3 (Drinfeld, [Dri86])

Die folgenden beiden Kategorien sind äquivalent:

I) Kategorie der $\mathcal{O}_{X \times S}$-Vektorbündel $\mathscr{E}$ von Rang $\frac{d}{\operatorname{deg}(\infty)}$, von allgemeinen Typ und mit Euler-Charakteristik $\chi\left(\mathscr{E}_{s}\right)=0$ für alle $s \in S$. 
II) Kategorie der $\mathcal{O}_{S}$-Moduln $\mathscr{M}$ mit einer aufsteigenden Filtrierung von $\mathcal{O}_{S}-$ Moduln $\mathscr{M}_{i}$ und einem Ringhomomorphismus $A \longrightarrow \operatorname{End}_{\mathcal{O}_{S}}(\mathscr{M})$ mit:

a) Es ist $\lim _{\longrightarrow} \mathscr{M}_{i}=\mathscr{M}$.

b) Für alle $i \in \mathbb{Z}$ sind die $\mathcal{O}_{S}$-Moduln $\mathscr{M}_{i}$ Vektorbündel von Rang $\max (d i, 0)$. Die $\mathcal{O}_{S}$-Moduln $\mathscr{M}_{i} / \mathscr{M}_{i-1}$ sind Vektorbündel von Rang $d$ für $i>0$, von Rang 0 für $i \leq 0$.

c) Für alle $a \in A, a \neq 0$ ist $a \mathscr{M}_{i} \subseteq \mathscr{M}_{i+\operatorname{deg}(a)}$ und die Abbildung

$$
\mathscr{M}_{i+1} / \mathscr{M}_{i} \stackrel{a \cdot}{\longrightarrow} \mathscr{M}_{i+1+\operatorname{deg}(a)} / \mathscr{M}_{i+\operatorname{deg}(a)}
$$

ist ein Isomorphismus für alle $i \geq 0$.

\subsection{Kategorie I $\Longrightarrow$ Kategorie II}

Sei $\mathscr{E}$ eine $\mathcal{O}_{X \times S^{-}}$Modulgarbe von allgemeinem Typ und für alle $i \in \mathbb{Z}$ sei $\mathscr{E}_{i}:=\mathscr{E}(i \infty)$. Wir definieren für alle $i \in \mathbb{Z}$ die $\mathcal{O}_{S^{-}}$Moduln

$$
\mathscr{M}_{i}:=\operatorname{pr}_{S *} \mathscr{E}_{i} \text { und } \mathscr{M}:=\operatorname{pr}_{S *}\left(\left.\mathscr{E}\right|_{\operatorname{spec} A \times S}\right) .
$$

\section{Bemerkung 4.4}

Für $i \leq j$ definieren die kanonischen Inklusionen

$$
\mathcal{O}_{X}(i \infty) \hookrightarrow \mathcal{O}_{X}(j \infty)
$$

ein induktives System von Abbildungen

$$
\mathscr{E}_{i} \longrightarrow \mathscr{E}_{j}
$$

Die Anwendung des Funktors $\mathrm{pr}_{S *}$ induziert also injektive Abbildungen

$$
\mathscr{M}_{i}=\operatorname{pr}_{S *} \mathscr{E}_{j} \longrightarrow \operatorname{pr}_{S *} \mathscr{E}_{j}=\mathscr{M}_{j}
$$

Diese definieren ein induktives System.

\section{Lemma 4.5}

Für alle $i \in \mathbb{Z}$ gilt $\left.\mathscr{E}_{i}\right|_{\operatorname{spec} A \times S}=\left.\mathscr{E}\right|_{\operatorname{spec} A \times S}$.

Beweis Sei $\iota: \operatorname{spec} A \times S \longrightarrow X \times S$ die kanonische Einbettung. Es ist

$$
\begin{aligned}
\left.\mathscr{E}_{i}\right|_{\operatorname{spec} A \times S} & \left.\left.\cong \mathscr{E}\right|_{\operatorname{spec} A \times S} \otimes_{\mathcal{O}_{\mathrm{spec} A \times S}}\left(\operatorname{pr}_{X}^{*} \mathcal{O}_{X}(i \infty)\right)\right|_{\mathrm{spec} A \times S} \\
& \left.\cong \mathscr{E}\right|_{\operatorname{spec} A \times S} \otimes_{\mathcal{O}_{\mathrm{spec} A \times S}} \operatorname{pr}_{\mathrm{spec} A}^{*}\left(\left.\mathcal{O}_{X}(i \infty)\right|_{\operatorname{spec} A}\right) \\
& \left.\cong \mathscr{E}\right|_{\operatorname{spec} A \times S} \otimes_{\mathcal{O}_{\mathrm{spec} A \times S}} \operatorname{pr}_{\mathrm{spec} A}^{*} \mathcal{O}_{\mathrm{spec} A} \\
& \left.\cong \mathscr{E}\right|_{\operatorname{spec} A \times S}
\end{aligned}
$$




\section{Folgerung 4.6}

Es gilt $\lim _{\longrightarrow} \mathscr{M}_{i} \cong \mathscr{M}$.

Beweis Es ist

$$
\begin{array}{rlr}
\lim _{\longrightarrow} \mathscr{M}_{i} & \cong \lim _{\lim _{S *} \mathscr{E}_{i}} & \\
& \cong \operatorname{pr}_{S *}{\stackrel{\lim }{\longrightarrow} \mathscr{E}_{i}} \quad(\text { Satz 2.70) } \\
& \left.\cong \operatorname{pr}_{S *} \iota_{*} \mathscr{E}\right|_{\operatorname{spec} A \times S} \quad(\text { Satz 2.70) } \\
& \left.\cong \operatorname{pr}_{S *} \mathscr{E}\right|_{\text {spec } A \times S} \\
& \cong \mathscr{M} .
\end{array}
$$

Wir definieren im Folgenden die $A$-Modul-Struktur auf dem $\mathcal{O}_{S^{-}}$Modul $\mathscr{M}$.

\section{Bemerkung 4.7}

1) Für alle $i, j \in \mathbb{Z}$ definieren die kanonischen Isomorphismen

$$
\mathcal{O}_{X}(i \infty) \otimes_{\mathcal{O}_{X}} \mathcal{O}_{X}(j \infty) \longrightarrow \mathcal{O}_{X}((i+j) \infty)
$$

Isomorphismen

$$
\operatorname{pr}_{X}^{*} \mathcal{O}_{X}(i \infty) \otimes_{\mathcal{O}_{X \times S}} \mathscr{E}_{j} \longrightarrow \mathscr{E}_{i+j}
$$

2) Sei $a \in A$. Für alle $j \geq \operatorname{deg}(a)$ gilt $a \in \mathcal{O}_{X}(j \infty)(X)$. Insbesondere vermittelt das Element $a$ mit Hilfe der Abbildungen aus Teil 1) für alle $j \geq \operatorname{deg}(a)$ die $\mathcal{O}_{X}$-linearen injektiven Abbildungen

$$
\mathcal{O}_{X}(i \infty) \stackrel{a \cdot}{\longrightarrow} \mathcal{O}_{X}((i+j) \infty)
$$

und damit $\mathcal{O}_{X \times S^{-}}$lineare injektive Abbildungen

$$
\mathscr{E}_{i} \stackrel{a \cdot}{\longrightarrow} \mathscr{E}_{i+j}
$$

Anwendung des Funktors $\mathrm{pr}_{S *}$ induziert injektive Abbildungen

$$
\mathscr{M}_{i} \cong \operatorname{pr}_{S *} \mathscr{E}_{i} \stackrel{a \cdot}{\longrightarrow} \operatorname{pr}_{S *} \mathscr{E}_{i+j} \cong \mathscr{M}_{i+j}
$$

3) Die Abbildungen aus Teil 2) sind kompatibel mit dem induktiven System auf den Garben $\mathscr{M}_{i}$. Sie definieren $\mathcal{O}_{S^{-}}$lineare Abbildungen auf dem Modul $\mathscr{M}$.

4) $\left.\mathrm{Da} \mathscr{M} \cong \operatorname{pr}_{S *} \mathscr{E}\right|_{\operatorname{spec} A \times S}$ gilt, gibt es in kanonischer Weise eine $A$-ModulStruktur auf dem $\mathcal{O}_{S^{-}}$Modul $\mathscr{M}$. Diese stimmt mit der in Teil 3) definierten $A$-Modul-Struktur überein. 


\section{Lemma 4.8}

Die Garbe $\mathscr{M}$ ist ein flacher $\mathcal{O}_{S}-$ Modul.

Beweis Die Aussage ist lokal in $S$ ([Liu02], Kapitel 5, Lemma 2.31, Seite 190). Sei $S=\operatorname{spec} R$, und sei $M:=H^{0}(S, \mathscr{M})$. Aus dem Beweis von Folgerung 4.6 entnehmen wir

$$
M=H^{0}(S, \mathscr{M})=H^{0}\left(\operatorname{spec} A \times S,\left.\mathscr{E}\right|_{\operatorname{spec} A \times S}\right) .
$$

Als Rückzug des $\mathcal{O}_{X \times S^{-}}$Vektorbündels $\mathscr{E}$ ist auch $\left.\mathscr{E}\right|_{\operatorname{spec} A \times S}$ ein $\mathcal{O}_{\text {spec } A \times S^{-}}$ Vektorbündel. Nach Voraussetzung ist spec $A \times S=\operatorname{spec} A \otimes R$ ein affines Schema. Der $A \otimes R$-Modul $H^{0}\left(\operatorname{spec} A \times S,\left.\mathscr{E}\right|_{\operatorname{spec} A \times S}\right)$ ist deshalb projektiv, insbesondere flach. Die Aussage folgt nun aus Lemma 2.6.

\section{Satz 4.9}

Sei $\mathscr{E}$ ein Vektorbündel von allgemeinem Typ über einem Körper K, von Rang $\frac{d}{\operatorname{deg}(\infty)}$ und mit Euler-Charakteristik $\chi(\mathscr{E})=0$. Sei $n_{0}$ maximal mit $H^{0}\left(X, \mathscr{E}_{n_{0}}\right)=0$, dann ist $n_{0}=0$.

Zum Beweis des Satzes benötigen wir das folgende Lemma.

\section{Lemma 4.10}

Es gilt $\chi\left(\mathscr{E} \otimes_{\mathcal{O}_{X}} \mathcal{O}_{X}(\infty)\right)=\chi(\mathscr{E})+d$.

Beweis Siehe [LRS93], (2.9).

\section{Folgerung 4.11}

Für alle $i \in \mathbb{Z}$ gilt $\chi\left(\mathscr{E}_{i}\right)=i d$.

Beweis (von Satz 4.9) Wir betrachten die für alle $i \in \mathbb{Z}$ exakte Sequenz

$$
\begin{aligned}
0 \longrightarrow H^{0}\left(X, \mathscr{E}_{i}\right) \longrightarrow H^{0}\left(X, \mathscr{E}_{i+1}\right) \longrightarrow H^{0}\left(X, \mathscr{E}_{i+1} / \mathscr{E}_{i}\right) \longrightarrow \\
\longrightarrow H^{1}\left(X, \mathscr{E}_{i}\right) \longrightarrow H^{1}\left(X, \mathscr{E}_{i+1}\right) \longrightarrow 0
\end{aligned}
$$

Aus der Injektivität der ersten Abbildung und der Surjektivität der letzten Abbildung folgt

$$
h^{0}\left(X, \mathscr{E}_{i}\right) \leq h^{0}\left(X, \mathscr{E}_{i+1}\right) \text { und } h^{1}\left(X, \mathscr{E}_{i}\right) \geq h^{1}\left(X, \mathscr{E}_{i+1}\right)
$$

Nach Folgerung 2.66 gilt $h^{0}\left(X, \mathscr{E}_{i+1} / \mathscr{E}_{i}\right)=d$. Aus der obigen Sequenz folgt dann

$$
h^{0}\left(X, \mathscr{E}_{i}\right)-h^{0}\left(X, \mathscr{E}_{i+1}\right)+d \underbrace{-h^{1}\left(X, \mathscr{E}_{i}\right)+h^{1}\left(X, \mathscr{E}_{i+1}\right)}_{\leq 0}=0
$$


Also folgt

$$
h^{0}\left(X, \mathscr{E}_{i}\right)-h^{0}\left(X, \mathscr{E}_{i+1}\right)+d \geq 0
$$

und insgesamt

$$
0 \leq h^{0}\left(X, \mathscr{E}_{i+1}\right)-h^{0}\left(X, \mathscr{E}_{i}\right) \leq d .
$$

Insbesondere folgt nach Wahl von $n_{0}$, Lemma 4.10 und da $\mathscr{E}$ von allgemeinem Typ ist

$$
h^{0}\left(X, \mathscr{E}_{n_{0}+1}\right)-h^{0}\left(X, \mathscr{E}_{n_{0}}\right)=\left(n_{0}+1\right) d \leq d \quad \Longrightarrow \quad n_{0} \leq 0 .
$$

Aus $\chi\left(\mathscr{E}_{n_{0}+1}\right)=h^{0}\left(\mathscr{E}_{n_{0}+1}\right)=\left(n_{0}+1\right) d>0$ folgt $n_{0} \geq 0$. Es folgt die Behauptung.

\section{Bemerkung 4.12}

Ist in Satz $4.9 \chi(\mathscr{E})=l$ für ein $l \in \mathbb{Z}$, dann ergibt die obige Rechnung

$$
0<l+\left(n_{0}+1\right) d \leq d
$$

und es folgt $n_{0}=\left\lceil\frac{-l}{d}\right\rceil$.

Folgerung 4.13

Da nach Definition $\mathscr{E}_{0}=\mathscr{E}$ ist, folgt

$$
\left.H^{0}\left(X \times s, \mathscr{E}_{s}\right)=H^{1}\left(X \times s, \mathscr{E}_{s}\right)\right)=0 \quad \forall s \in S .
$$

\section{Satz 4.14}

Sei $S$ ein lokal noethersches Schema. Dann sind für alle $i \in \mathbb{Z}$ die $\mathcal{O}_{S}-$ Moduln $R^{0} \operatorname{pr}_{S *} \mathscr{E}_{i}$ und $R^{1} \operatorname{pr}_{S *} \mathscr{E}_{i}$ projektiv.

Beweis Es reicht aus, die Aussage für $S=\operatorname{spec} R$ zu zeigen. Nach Theorem 2.45 folgt aus

$$
H^{k}\left(X \times s, \mathscr{E}_{s}\right)=0 \quad \forall s \in S
$$

auch

$$
H^{k}(X \times S, \mathscr{E})=0
$$

für $k=0,1$. Aus der langen Kohomologiesequenz

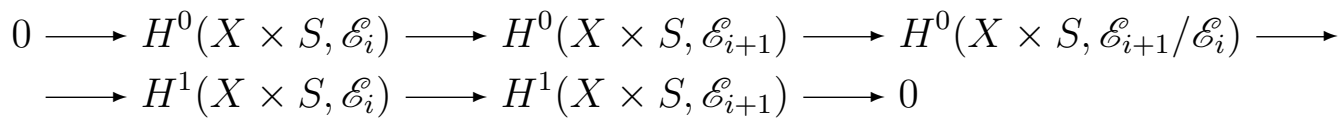

ergibt sich für $i=0$

$$
H^{0}\left(X, \mathscr{E}_{1}\right) \cong H^{0}\left(X, \mathscr{E}_{1} / \mathscr{E}_{0}\right)
$$


Nach Folgerung 2.66 ist für alle $i \in \mathbb{Z}$ der $R$-Modul $H^{0}\left(X, \mathscr{E}_{i+1} / \mathscr{E}_{i}\right)$ projektiv von Rang $d$.

Analog ergibt sich für $i=-1$

$$
H^{0}\left(X, \mathscr{E}_{0} / \mathscr{E}_{-1}\right) \cong H^{1}\left(X, \mathscr{E}_{-1}\right)
$$

Für $i>0$ ist die Sequenz

$$
0 \longrightarrow H^{0}\left(X, \mathscr{E}_{i}\right) \longrightarrow H^{0}\left(X, \mathscr{E}_{i+1}\right) \longrightarrow H^{0}\left(X, \mathscr{E}_{i+1} / \mathscr{E}_{i}\right) \longrightarrow 0
$$

exakt und spaltend. Da die direkte Summe zweier projektiver Moduln wieder projektiv ist, ergibt sich die Behauptung induktiv.

Analog ist für $i<0$ die Sequenz

$$
0 \longrightarrow H^{0}\left(X, \mathscr{E}_{i+1} / \mathscr{E}_{i}\right) \longrightarrow H^{1}\left(X, \mathscr{E}_{i}\right) \longrightarrow H^{1}\left(X, \mathscr{E}_{i+1}\right) \longrightarrow 0
$$

exakt. Die Behauptung ergibt sich wieder durch Induktion.

\section{Satz 4.15}

Sei $S$ ein beliebiges Schema. Dann sind für alle $i \in \mathbb{Z}$ die $\mathcal{O}_{S}$-Moduln $R^{0} \operatorname{pr}_{S *} \mathscr{E}_{i}$ und $R^{1} \operatorname{pr}_{S *} \mathscr{E}_{i}$ projektiv.

Beweis Es reicht, die Aussage für $S=\operatorname{spec} R$ zu zeigen. Mit Hilfe von Satz 4.14 können wir die Ergebnisse von Abschnitt 2.6 anwenden. Die Voraussetzungen von Satz 2.49 und Satz 2.50 sind erfüllt. Wir können folglich den Beweis von Folgerung 4.14 auch auf die Situation eines beliebigen Ringes $R$ übertragen.

\section{Folgerung 4.16}

Für alle $i \in \mathbb{Z}$ sind die $\mathcal{O}_{S}$-Moduln $R^{0} \operatorname{pr}_{S *} \mathscr{E}_{i}$ und $R^{1} \mathrm{pr}_{S *} \mathscr{E}_{i}$ Vektorbündel und es gilt

$$
\operatorname{rk}\left(R^{0} \operatorname{pr}_{S *} \mathscr{E}_{i}\right)=\left\{\begin{array}{l}
i d \text { für } i \geq 0 \\
0 \text { für } i \leq 0
\end{array}\right.
$$

und

$$
\operatorname{rk}\left(R^{1} \operatorname{pr}_{S *} \mathscr{E}_{i}\right)=\left\{\begin{array}{l}
0 \text { für } i \geq 0 \\
-i d \text { für } i \leq 0
\end{array}\right.
$$

\section{Lemma 4.17}

Für alle $i \in \mathbb{Z}$ und alle $a \in A, a \neq 0$ sind die Abbildungen

$$
\mathcal{O}_{X}(i \infty) / \mathcal{O}_{X}((i-1) \infty) \stackrel{a \cdot}{\longrightarrow} \mathcal{O}_{X}((i+\operatorname{deg}(a)) \infty) / \mathcal{O}_{X}((i-1+\operatorname{deg}(a)) \infty)
$$

Isomorphismen. 
Beweis Die Aussage lässt sich halmweise auf den abgeschlossenen Punkten von $X$ testen. Sei $x \in X$ ein abgeschlossener Punkt. Für $x \neq \infty$ ist $\mathcal{O}_{X}(i \infty)_{x}=\mathcal{O}_{X, x}$ und es ist nichts zu zeigen. Sei $\pi_{\infty} \in \mathcal{O}_{X, \infty}$ ein uniformisierendes Element. Es gibt ein $\varepsilon \in \mathcal{O}_{X, \infty}^{\star}$ mit $a=\varepsilon \pi^{\infty(a)}$. Es gilt dann

$$
\mathcal{O}_{X}(i \infty)_{\infty}=\pi_{\infty}^{-i} \mathcal{O}_{X, \infty}
$$

und für alle $i \in \mathbb{Z}$ sind die Abbildungen

$$
\pi^{-i} \mathcal{O}_{X, \infty} / \pi^{-i+1} \mathcal{O}_{X, \infty} \stackrel{\varepsilon \pi^{\infty(a)}}{\longrightarrow} \pi^{-i+\infty(a)} \mathcal{O}_{X, \infty} / \pi^{-i+1+\infty(a)} \mathcal{O}_{X, \infty}
$$

Isomorphismen.

Folgerung 4.18

Für alle $i \geq 0$ und alle $a \in A, a \neq 0$ sind die Abbildungen

$$
\operatorname{pr}_{S *} \mathscr{E}_{i+1} / \operatorname{pr}_{S *} \mathscr{E}_{i} \stackrel{a \cdot}{\longrightarrow} \operatorname{pr}_{S *} \mathscr{E}_{i+1+\operatorname{deg}(a)} / \operatorname{pr}_{S *} \mathscr{E}_{i+\operatorname{deg}(a)}
$$

Isomorphismen.

Beweis Nach Lemma 4.17 vermittelt die Multiplikation mit $a$ für alle $i \in \mathbb{Z}$ Isomorphismen

$$
\mathscr{E}_{i+1} / \mathscr{E}_{i} \stackrel{a \cdot}{\longrightarrow} \mathscr{E}_{i+1+\operatorname{deg}(a)} / \mathscr{E}_{i+\operatorname{deg}(a)}
$$

Für $i \geq 0$ gilt $R^{1} \operatorname{pr}_{S *} \mathscr{E}_{i}=0$ und es folgt

$$
\operatorname{pr}_{S *} \mathscr{E}_{i+1} / \operatorname{pr}_{S *} \mathscr{E}_{i}=\operatorname{pr}_{S *}\left(\mathscr{E}_{i+1} / \mathscr{E}_{i}\right)
$$

Es folgt die Behauptung.

\subsection{Kategorie II $\Longrightarrow$ Kategorie I}

Wir betrachten zunächst den affinen Fall $S=\operatorname{spec} R$. Die Daten der Kategorie II bedeuten dann:

Sei $M$ ein $A \otimes R$-Modul mit einer aufsteigenden Filtrierung von $R$-Moduln $M_{i}$ mit:

a) Es ist $\lim _{\longrightarrow} M_{i}=M$.

b) Für alle $i \in \mathbb{Z}$ sind die $R$-Moduln $M_{i}$ projektiv von $\operatorname{Rang} \max (d i, 0)$ und die Moduln $M_{i+1} / M_{i}$ sind projektiv von Rang $d$ für $i \geq 0$, von Rang 0 für $i<0$. 
c) Für alle $a \in A, a \neq 0$ ist $a M_{i} \subseteq M_{i+\operatorname{deg}(a)}$ und die Abbildung

$$
M_{i+1} / M_{i} \stackrel{a \cdot}{\longrightarrow} M_{i+1+\operatorname{deg}(a)} / M_{i+\operatorname{deg}(a)}
$$

ist ein Isomorphismus für alle $i \geq 0$.

\section{Bemerkung 4.19}

Die Daten der Kategorie II sind verträglich mit Basiswechsel in $R$.

Nach Satz 2.37 und mit Hilfe des flachen Basiswechsels $S / \mathbb{F}_{q}$ gilt

$$
\begin{aligned}
\operatorname{Proj}\left(\bigoplus_{i=0}^{\infty} H^{0}\left(X \times S, \mathcal{O}_{X \times S}(i \infty)\right)\right. & \cong \operatorname{Proj}\left(\bigoplus_{i=0}^{\infty} H^{0}\left(X, \mathcal{O}_{X}(i \infty)\right) \otimes R\right) \\
& \cong X \times S
\end{aligned}
$$

Sei $\mathcal{S}_{X}:=\bigoplus_{i=0}^{\infty} H^{0}\left(X, \mathcal{O}_{X}(i \infty)\right)$. Der Modul $\mathcal{M}:=\bigoplus_{i=0}^{\infty} M_{i}$ ist in kanonischer Weise ein graduierter $\mathcal{S}_{X} \otimes R$-Modul, und wir konstruieren mit Hilfe des Proj-Funktors eine $\mathcal{O}_{X \times S^{-}}$Modulgarbe $\mathscr{E}$. Wir zeigen im Folgenden, dass sie den Bedingungen der Kategorie I genügt.

Satz 4.20

Der $\mathcal{O}_{X \times S}-$ Modul $\mathcal{M}^{\sim}$ ist ein Vektorbündel von allgemeinem Typ und von Rang $d / \operatorname{deg}(\infty)$.

Zum Beweis des Satzes zeigen wir im Folgenden zunächst, dass im Falle eines reduzierten, noetherschen Ringes $R$ der $A \otimes R$-Modul $\left.\mathcal{M}\right|_{\text {spec } A \times S}$ projektiv und endlich erzeugt ist und berechnen seinen Rang (4.22 -4.28). Dann zeigen wir dieselbe Aussage für $\left.\mathcal{M}\right|_{\text {spec } k(\infty) \times S}$ (4.29). Beide Aussagen zusammen ergeben mit Hilfe der Konstanz der Faserdimension (Satz 2.72) die Aussage von Satz 4.20 im Falle eines reduzierten, noetherschen Ringes $R$. Den Fall eines beliebigen, noetherschen Ringes $R$ zeigen wir in 4.31 mit Hilfe von Theorem 2.9. Für den allgemeinen Fall konstruieren wir in 4.32 explizit ein Abstiegsdatum.

\section{Lemma 4.21}

Der $R$-Modul $\mathcal{M}$ ist projektiv.

Beweis Als direkte Summe projektiver $R$-Moduln ist $\mathcal{M}=\bigoplus_{i=0}^{\infty} M_{i}$ auch ein projektiver $R$-Modul.

\section{Lemma 4.22}

$\operatorname{Der} \mathcal{S}_{X} \otimes R$-Modul $\mathcal{M}$ ist endlich erzeugt. 
Beweis Für alle $i \geq 0$ spaltet nach Voraussetzung die Sequenz

$$
0 \longrightarrow M_{i} \longrightarrow M_{i+1} \longrightarrow M_{i+1} / M_{i} \longrightarrow 0
$$

und es folgt

$$
M_{i+1} \cong \bigoplus_{k=0}^{i} M_{k+1} / M_{k} .
$$

Sei $0 \neq a \in H^{0}\left(X, \mathcal{O}_{X}(\operatorname{deg}(a) \infty) \subset A\right.$. Dann gilt nach Voraussetzung für alle $i \geq 0$

$$
M_{i+1} / M_{i} \cong M_{i+1+\operatorname{deg} a} / M_{i+\operatorname{deg} a} .
$$

Die $R$-Moduln $M_{0}, \ldots, M_{\operatorname{deg} a}$ erzeugen folglich $\mathcal{M}$ als $\mathcal{S}_{X} \otimes R$-Modul. Da die Moduln $M_{0}, \ldots, M_{\operatorname{deg} a}$ nach Voraussetzung endlich erzeugte $R$-Moduln sind, folgt die Behauptung.

Wir betrachten nun die Einschränkung von $\mathcal{M}^{\sim}$ auf $\operatorname{spec} A \times S$.

\section{Lemma 4.23}

Es gilt

$$
\left.(\mathcal{M})^{\sim}\right|_{\operatorname{spec} A \times \operatorname{spec} R} \cong M^{\sim} .
$$

Beweis Mit den Bezeichnungen aus Folgerung 2.39 betrachten wir die Abbildung

$$
\begin{aligned}
\left(\mathcal{M}\left[e^{-1}\right]\right)_{(0)} & \longrightarrow M \\
\frac{m}{e^{i}} & \longmapsto m .
\end{aligned}
$$

Diese ist ein Isomorphismus.

\section{Lemma 4.24}

Der R-Modul $M$ ist projektiv.

Beweis Für alle $i \geq 0$ spaltet nach Voraussetzung die Sequenz

$$
0 \longrightarrow M_{i} \longrightarrow M_{i+1} \longrightarrow M_{i+1} / M_{i} \longrightarrow 0
$$

und es folgt

$$
M_{i+1} \cong \bigoplus_{k=0}^{i} M_{k+1} / M_{k}
$$

Übergang zum Limes liefert

$$
M \cong \bigoplus_{k=0}^{\infty} M_{k+1} / M_{k}
$$

Als direkte Summe projektiver $R$-Moduln ist $M$ ein projektiver $R$-Modul. 


\section{Lemma 4.25}

Der $R \otimes A$-Modul $M$ ist endlich erzeugt.

Beweis Sei $a \in A$ mit $\operatorname{deg} a \neq 0$. Nach Voraussetzung gilt für alle $i \geq 0$

$$
M_{i+1} / M_{i} \cong M_{i+1+\operatorname{deg} a} / M_{i+\operatorname{deg} a}
$$

und mit Hilfe der Zerlegung aus Lemma 4.24 folgt, dass $M$ als $R \otimes A$-Modul bereits von $M_{1} / M_{0}, \ldots, M_{\operatorname{deg} a} / M_{\operatorname{deg} a-1}$ erzeugt wird. Diese sind nach Voraussetzung endlich erzeugte $R$-Moduln. Es folgt die Behauptung.

\section{Definition 4.26}

Sei

$$
\begin{aligned}
\operatorname{deg}: M & \longrightarrow \mathbb{Z} \\
m & \longmapsto \min \left\{i \mid m \notin M_{i-1}\right\} .
\end{aligned}
$$

\section{Lemma 4.27}

Sei $0 \neq a \in A$. Dann gilt $\operatorname{deg}(a m)=\operatorname{deg} a+\operatorname{deg} m$.

Beweis Sei $\operatorname{deg} m=i$. Dann ist $m \notin M_{i-1}$. Da die Abbildung

$$
M_{i} / M_{i-1} \stackrel{a}{\longrightarrow} M_{i+\operatorname{deg} a} / M_{i-1+\operatorname{deg} a}
$$

nach Voraussetzung ein Isomorphismus ist, folgt $a m \in M_{i+\operatorname{deg} a}$ und $a m \notin$ $M_{i+\operatorname{deg} a-1}$. Es folgt die Behauptung.

\section{Satz 4.28}

Der Modul $M$ ist ein projektiver $R \otimes A$-Modul von Rang $\frac{d}{\operatorname{deg}(\infty)}$.

Zum Beweis des Satzes gehen wir wie im Beweis von Satz 3.8 vor.

\subsection{1 $\mathrm{R}=\mathrm{L}$ ein Körper und $\mathrm{A}=\mathbb{F}_{\mathbf{q}}[\mathrm{T}]$}

Sei $T \in \mathbb{F}_{q}[T]$. Dann ist $\operatorname{deg} T=1$. Nach Lemma 4.25 ist der $L[T]$-Modul $M$ erzeugt von einer $L$-Basis des $L$-Vektorraumes $M_{1}$ und dieser hat nach Voraussetzung die Dimension $d$.

\subsection{2 $\mathrm{R}=\mathrm{L}$ ein Körper}

Die Behauptung ergibt sich analog zu Unterabschnitt 3.3.2. 


\subsection{3 $\mathrm{R}$ reduzierter, noetherscher Ring}

Die Behauptung ergibt sich analog zu Unterabschnitt 3.3.3.

Satz 4.29

$\left.\mathcal{M}\right|_{\operatorname{spec} k(\infty) \times S}$ ist ein projektiver $k(\infty) \otimes R$-Modul von Rang $\frac{d}{\operatorname{deg}(\infty)}$.

Beweis Sei $e:=1 \in H^{0}\left(X, \mathcal{O}_{X}(\infty)\right)$. Dann gilt

$$
\left.\mathcal{M}\right|_{\text {spec } k(\infty) \times S} \cong \mathcal{M} / e \mathcal{M} \cong \bigoplus_{i=0}^{\infty} M_{i+1} / M_{i}
$$

Nach Folgerung 2.40 gilt

$$
\operatorname{Proj}\left(\mathcal{S}_{X} \otimes R / e\left(\mathcal{S}_{X} \otimes R\right)\right) \cong \operatorname{spec} k(\infty) \otimes R .
$$

Sei $a \in A$ mit $\operatorname{deg} a>0$. Es gilt

$$
\operatorname{Proj}\left(\bigoplus_{i=0}^{\infty} M_{i+1} / M_{i}\right) \cong \operatorname{Proj}\left(\bigoplus_{i=0}^{\infty} M_{1+i \operatorname{deg} a} / M_{i \operatorname{deg} a}\right) \text {. }
$$

Für alle $i \geq 0$ gilt nach Voraussetzung

$$
M_{1} \cong M_{1+\operatorname{deg} a} / M_{\operatorname{deg} a} \cong M_{1+i \operatorname{deg} a} / M_{i \operatorname{deg} a}
$$

und es folgt

$$
\bigoplus_{i=0}^{\infty}\left(M_{1+i \operatorname{deg} a} / M_{i \operatorname{deg} a}\left[a^{-1}\right]\right)_{(0)} \cong M_{1} .
$$

$\mathrm{Zu}$ zeigen ist also, dass $M_{1}$ ein projektiver $k(\infty) \otimes R$-Modul von Rang $\frac{d}{\operatorname{deg}(\infty)}$ ist.

Nach Voraussetzung ist $M_{1}$ ein projektiver $R$-Modul von Rang $d$. Sei zunächst $R$ ein lokaler Ring. Dann ist $M_{1}$ ein freier $R$-Modul von Rang $d$. Sei $\mathfrak{p}$ das maximale Ideal von $R$ und sei $K:=R / \mathfrak{p}$. Es folgt, dass $\bar{M}_{1}:=M_{1} / \mathfrak{p} M_{1}$ ein $k(\infty) \otimes K$-Modul ist. Wir erhalten durch die $k(\infty)$-Operation auf $\bar{M}_{1}$ eine Einbettung

$$
k(\infty) \longrightarrow \operatorname{Aut}_{K}\left(\bar{M}_{1}\right) \simeq \mathrm{GL}_{d}(K) \stackrel{\operatorname{det}}{\longrightarrow} K .
$$

Da $k(\infty)$ eine endliche, separable Körpererweiterung von $\mathbb{F}_{q}$ ist, zerfällt also $k(\infty) \otimes K \cong \prod_{i=1}^{\operatorname{deg}(\infty)} K$ in ein endliches Produkt. Der $\left(\prod_{i=1}^{\operatorname{deg}(\infty)} K\right)$-Modul $\bar{M}_{1}$ zerfällt folglich mit Hilfe der Galois-Aktion von $\operatorname{Gal}\left(k(\infty) / \mathbb{F}_{q}\right)$ für $1 \leq$ $i \leq \operatorname{deg}(\infty)$ in äqui-dimensionale $K$-Vektorräume $V_{i}$ der Dimension $\frac{d}{\operatorname{deg}(\infty)}$. Insbesondere gilt

$$
\operatorname{rk}_{k(\infty) \otimes K} \bar{M}_{1}=\frac{d}{\operatorname{deg}(\infty)} .
$$

Für einen beliebigen Ring $R$ folgt die Behauptung nun aus der Konstanz der Faserdimension (Satz 2.72). 


\section{Folgerung 4.30}

Sei $R$ ein noetherscher, reduzierter Ring. Der $\mathcal{O}_{X \times S^{-}}$Modul Proj $\mathcal{M}$ ist lokal frei von Rang $\frac{d}{\operatorname{deg} \infty}$.

Beweis Die Behauptung ergibt sich aus der Konstanz der Faserdimension.

\section{Folgerung 4.31}

Sei $R$ ein noetherscher Ring. Der $\mathcal{O}_{X \times S}-$ Modul Proj $\mathcal{M}$ ist lokal frei von Rang $\frac{d}{\operatorname{deg} \infty}$.

Beweis Da $\mathcal{M}$ ein projektiver, insbesondere flacher, $R$-Modul ist, können wir das Theorem 2.9 aus Unterabschnitt 3.3.4 anwenden (vgl. [Mat86], § 22, Bemerkung zu Theorem 22.6, Seite 177f). Sei also $I:=\operatorname{Rad}(R)$. Dann folgt

$$
\mathcal{M} \otimes_{\mathcal{S}_{X} \otimes R} \mathcal{S}_{X} \otimes I \cong \mathcal{M} \otimes_{R} I \cong I M
$$

\subsubsection{R beliebiger Ring}

Den allgemeinen Fall führen wir durch Basiswechsel auf den noetherschen Fall zurück. Wir benötigen zunächst das folgende Lemma.

\section{Lemma 4.32}

Sei $R$ eine $K$-Algebra und sei $M$ ein projektiver, endlich erzeugter $R$-Modul von Rang d. Dann gibt es einen noetherschen Unterring $R^{\prime} \subseteq R$ und einen projektiven, endlich erzeugten $R^{\prime}-$ Modul $M^{\prime} \subseteq M$ mit

$$
M=R \otimes_{R^{\prime}} M^{\prime} \text { und } \operatorname{rang} M=\operatorname{rang} M^{\prime} .
$$

Beweis Sei $M$ erzeugt von $m_{1}, \ldots, m_{k} \in M$. Dann gibt es eine surjektive Abbildung

$$
\varphi: R^{k} \longrightarrow M, \quad e_{i} \longmapsto m_{i} .
$$

Da $M$ ein projektiver $R$-Modul ist, gibt es einen Schnitt $s: M \longrightarrow R^{k}$. Sei für $1 \leq i \leq k, s\left(m_{i}\right)=\left(\lambda_{i 1}, \ldots, \lambda_{i k}\right) \in R^{k}$ und sei $R^{\prime}:=K\left[\lambda_{11}, \ldots, \lambda_{k k}\right]$. Als endlich erzeugte $K$-Algebra ist $R^{\prime}$ noethersch. Sei $M^{\prime}$ der von $m_{1} \ldots, m_{k}$ erzeugte $R^{\prime}$-Modul in $M$. Wir betrachten die surjektive Abbildung

$$
\varphi^{\prime}: R^{\prime k} \longrightarrow M^{\prime}, \quad e_{i} \longmapsto m_{i} .
$$

Nach Konstruktion landet die Einschränkung des Schnittes $\left.s\right|_{M^{\prime}}$ in $R^{\prime k}$ und ist ein Schnitt der Abbildung $\varphi^{\prime}$. Insbesondere ist $M^{\prime}$ ein projektiver $R^{\prime}$-Modul. 
Aus dem Diagramm

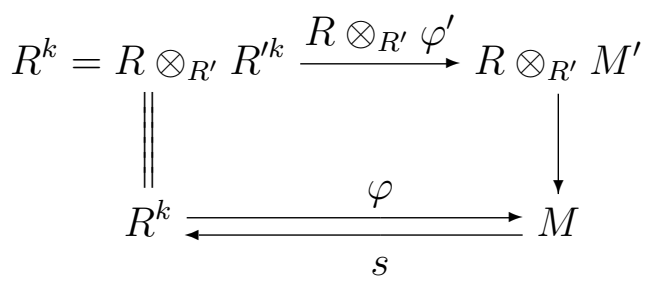

folgt die kanonische Isomorphie der $R$-Moduln $R \otimes_{R^{\prime}} M^{\prime} \cong M$.

Da $M^{\prime}$ ein endlich erzeugter, projektiver $R^{\prime}$-Modul ist, gibt es eine offene Überdeckung von spec $R^{\prime}=\bigcup U_{i}$, so dass rang $M^{\prime}=d^{\prime}$ konstant ist. Da $R^{\prime}$ ein Unterring von $R$ ist, hat die Abbildung spec $R \longrightarrow \operatorname{spec} R^{\prime}$ ein dichtes Bild. Insbesondere gibt es in jeder der offenen Mengen $U_{i}$ einen Bildpunkt $\mathfrak{p} \in \operatorname{spec} R^{\prime}$ eines Primideals $\mathfrak{P} \in \operatorname{spec} R$. Es folgt

$$
R_{\mathfrak{P}} \otimes_{R_{\mathfrak{p}}^{\prime}} M_{\mathfrak{p}}^{\prime} \cong M_{\mathfrak{P}}
$$

und $d^{\prime}=\operatorname{rang} M$.

Mit Hilfe von Lemma 4.32 können wir die Aussage von Satz 4.20 auf den noetherschen Fall zurückführen:

Nach Voraussetzung gibt es für alle $i \geq 1$ eine exakte Sequenz

$$
0 \longrightarrow M_{i} \longrightarrow M_{i+1} \longrightarrow M_{i+1} / M_{i} \longrightarrow 0
$$

projektiver $R$-Moduln $M_{i}$ von Rang $d i$. Der $R$-Modul $M_{i+1} / M_{i}$ ist projektiv von Rang $d$ für alle $i \in \mathbb{Z}$. Insbesondere spaltet die Sequenz und wir können für alle $i \in \mathbb{Z}$ den Modul $N_{i+1}:=M_{i+1} / M_{i}$ als direkten Summanden des Moduls $M_{i+1}$ auffassen. Nach Lemma 4.32 gibt es für alle $0<i \leq \operatorname{deg} a$ einen projektiven, endlich erzeugten $R^{\prime}$-Modul $N_{i}^{\prime}$ mit $R \otimes_{R^{\prime}} N_{i}^{\prime}=N_{i}$. Wir definieren induktiv $R^{\prime}$-Moduln $M_{i}^{\prime}$ wie folgt. Für $i=1$ wählen wir $M_{1}^{\prime}=N_{1}^{\prime}$. Für $i>1$ sei $M_{i}^{\prime}$ der von $N_{i}^{\prime}$ und $M_{i-1}^{\prime}$ erzeugte $R^{\prime}$-Modul in $M_{i}$. Wir erhalten für alle $i$ die exakte Sequenz

$$
0 \longrightarrow M_{i}^{\prime} \longrightarrow M_{i+1}^{\prime} \longrightarrow N_{i+1}^{\prime} \longrightarrow 0 \text {. }
$$

Induktiv folgt, dass die Sequenz spaltet und $M_{i}^{\prime}$ ein projektiver $R^{\prime}$-Modul von Rang $i d$ ist. Tensorieren der Sequenz mit $R \otimes_{R^{\prime}}$ ergibt

$$
R \otimes_{R^{\prime}} M_{i}^{\prime} \cong M_{i}
$$

Für $i>\operatorname{deg} a$, definieren wir die $R^{\prime}$-Moduln $M_{i}^{\prime}$ mit Hilfe der durch Multiplikation mit $a$ vermittelten Abbildungen. Die Konstruktion liefert einen 
$R^{\prime}$-Modul $M^{\prime}$ mit Filtrierung $M_{i}^{\prime}$ der Kategorie II. Basiswechsel liefert nun die Behauptung.

Damit ist Satz 4.20 bewiesen.

Abschließend zeigen wir, dass die Kohomologie von $\mathcal{M}^{\sim}$ die Bedingung eines Vektorbündels von allgemeinem Typ erfüllt.

Wir gehen analog zur Konstruktion in Abschnitt 3.4 vor. Zunächst setzen wir die Gradfunktion von $M$ auf $M \otimes_{A} F$ fort.

$$
\begin{aligned}
\operatorname{deg}: M \otimes_{A} F & \longrightarrow \mathbb{Z} \\
m \otimes x & \longmapsto \operatorname{deg} m+\operatorname{deg} x
\end{aligned}
$$

Dies ist nach Lemma 4.27 wohldefiniert.

Wir definieren für die Fasern in $\infty$ die $\mathcal{O}_{X, \infty} \otimes R$-Moduln für alle $i \in \mathbb{Z}$

$$
M_{i, \infty}:=\left\{\alpha \in M \otimes_{A} F \mid \operatorname{deg} \alpha \leq i\right\} .
$$

\section{Lemma 4.33}

Für alle $i \geq 0$ gilt

$$
M_{i+1} / M_{i} \cong M_{i+1, \infty} / M_{i, \infty} .
$$

Beweis Die kanonische Abbildung $M_{i} \longrightarrow M_{i, \infty}$ ist injektiv, es ist $M_{i+1} \cap$ $M_{i, \infty}=M_{i}$ und wir erhalten einen injektiven $R$-linearen Morphismus

$$
M_{i+1} / M_{i} \longleftrightarrow M_{i+1, \infty} / M_{i, \infty} .
$$

$\mathrm{Zu}$ zeigen ist nun die Surjektivität. Sei $m \otimes x \in M_{i+1, \infty} \backslash M_{i, \infty}$ mit $x=\frac{a}{b}$ und $0 \neq a, b \in A$. Dann ist $\operatorname{deg} m=i-\operatorname{deg} x$ und es folgt

$$
a m \in M_{i-\operatorname{deg} x+\operatorname{deg} a, \infty}=M_{i+\operatorname{deg} b, \infty} .
$$

Nach Voraussetzung ist

$$
M_{i} / M_{i-1} \stackrel{b}{\longrightarrow} M_{i+\operatorname{deg} b} / M_{i+\operatorname{deg} b-1}
$$

ein Isomorphismus. Es gibt deshalb ein Element $\tilde{m} \in M_{i}$ mit

$$
b \tilde{m} \equiv a m \quad \bmod M_{i+\operatorname{deg} b-1} .
$$

Es folgt

$$
\begin{array}{cc}
b \tilde{m} \otimes \frac{1}{b}-a m \otimes \frac{1}{b} \equiv 0 & \bmod M_{i-1, \infty} \\
\Longrightarrow \quad \tilde{m} \otimes 1 \equiv m \otimes \frac{a}{b} \quad \bmod M_{i-1, \infty} .
\end{array}
$$

Es folgt die Behauptung. 


\section{Lemma 4.34}

Sei $\mathfrak{p}_{\infty} \subseteq \mathcal{S}_{X}$ das zum Punkt $\infty \in X$ gehörende (homogene) Primideal. Dann gibt es für alle $k \in \mathbb{Z}$ einen kanonischen $\mathcal{O}_{X, \infty} \otimes R$-linearen Isomorphismus

$$
(\mathcal{M}[k])_{\left(\mathfrak{p}_{\infty}\right)} \cong M_{k, \infty}
$$

Beweis Wir betrachten die Abbildung

$$
\begin{aligned}
& M \otimes_{A} F \longrightarrow(\mathcal{M}[k])_{\left(\mathfrak{p}_{\infty}\right)} \\
& m \otimes \frac{1}{f} \longmapsto \frac{m}{f} .
\end{aligned}
$$

Die Abbildung induziert den gewünschten Isomorphismus.

\section{Folgerung 4.35}

Sei $\mathscr{E}_{k}:=\operatorname{Proj}(\mathcal{M}[k])$. Dann gilt

$$
\left.\mathscr{E}_{k}\right|_{\operatorname{spec} \mathcal{O}_{X, \infty} \times S} \cong M_{k, \infty}^{\sim}
$$

\section{Folgerung 4.36}

Für alle $i \in \mathbb{Z}$ gilt

$$
\mathscr{E}_{i}(X \times S)=\{m \in M \mid \operatorname{deg} m \leq i\} .
$$

\section{Folgerung 4.37}

Es gilt:

1) $H^{0}\left(X \times S, \mathscr{E}_{i}\right)=H^{0}\left(X \times s,\left(\mathscr{E}_{i}\right)_{s}\right)=0$ für $i \leq 0$ und $s \in S$.

2) $H^{1}\left(X \times S, \mathscr{E}_{i}\right)=H^{1}\left(X \times s,\left(\mathscr{E}_{i}\right)_{s}\right)=0$ für $i \geq 0$ und $s \in S$.

3) $\lim _{i \in \mathbb{Z}} H^{0}\left(X \times S, \mathscr{E}_{i}\right)=M$

\section{Beweis}

1) Folgt aus Folgerung 4.36.

2) Nach Lemma 2.64 hat $\mathscr{E}_{i} / \mathscr{E}_{i-1}$ nur Träger in $\operatorname{spec} \mathcal{O}_{X, \infty} \otimes S$. Nach Lemma 4.33 ist für alle $i \geq 0$

$$
M_{i+1} / M_{i} \cong M_{i+1, \infty} / M_{i, \infty}
$$

ein Isomorphismus von $R$-Moduln. Nach Voraussetzung ist $M_{i+1} / M_{i}$ endlich erzeugt und folglich wird $M_{i+1, \infty} / M_{i, \infty}$ von globalen Schnitten erzeugt. Es folgt, dass die kanonische Abbildung

$$
H^{0}\left(X \times S, \mathscr{E}_{i+1}\right) \longrightarrow H^{0}\left(X \times S, \mathscr{E}_{i+1} / \mathscr{E}_{i}\right)
$$

für alle $i \geq 0$ surjektiv ist. Insbesondere ist $H^{1}\left(X \times S, \mathscr{E}_{i}\right)=0$ für alle $i \geq 0$. 
3) Folgt aus Folgerung 4.36.

\section{Bemerkung 4.38}

Wählen wir in Satz 4.3 allgemeiner $\chi(\mathscr{E})=l$ für ein $l \in \mathbb{Z}$, so ergibt sich für Kategorie II die Bedingung $\operatorname{rk}\left(M_{i}\right)=\max (l+i d, 0)$ und für alle $0 \neq a \in A$ die Isomorphie

$$
M_{i} / M_{i-1} \stackrel{a}{\longrightarrow} M_{i+\operatorname{deg} a} / M_{i-1+\operatorname{deg} a}
$$

für alle nicht verschwindenden Quotienten $M_{i} / M_{i-1}$.

\section{Bemerkung 4.39}

Ist $S$ ein beliebiges Schema, dann ist

$$
\bigoplus_{i=0}^{\infty} \mathscr{M}_{i}
$$

ein graduierter $\mathcal{S}_{X} \otimes \mathcal{O}_{S}$-Modul. Die Proj-Konstruktion definiert einen $\mathcal{O}_{X \times S^{-}}$ Modul $\mathscr{E}$. Da wir die Daten von Kategorie I auf einer affinen, offenen Überdeckung von $S$ testen können, ist nach dem bereits Gezeigten $\mathscr{E}$ ein $\mathcal{O}_{X \times S^{-}}$ Vektorbündel von allgemeinem Typ.

\subsection{Kategorienäquivalenz}

Wir fassen Kategorie I als eine Unterkategorie der Kategorie der quasikohärenten $\mathcal{O}_{X \times S^{-}}$Moduln und Kategorie II als Unterkategorie der Kategorie der graduierten $\mathcal{S}_{X \times S^{-}}$Moduln auf.

Die Konstruktion in Abschnitt 4.3 wird durch die Anwendung des Funktors $\Gamma(\cdot)_{*}$ durchgeführt und ist folglich funktoriell und die Konstruktion in Abschnitt 4.4 wird durch die Anwendung des Funktors $(\cdot)^{\sim}$ durchgeführt und ist also ebenfalls funktoriell.

Nach [GD61], Kapitel II, 3.3, Seite 56ff, gibt es kanonische Transformationen

$$
\begin{aligned}
& \alpha:(\cdot) \longrightarrow \Gamma_{*}(\cdot \sim) \\
& \beta: \Gamma_{*}(\cdot)^{\sim} \longrightarrow(\cdot)
\end{aligned}
$$

für die Komposition der Funktoren $\Gamma_{*}(\cdot)$ und $(\cdot)^{\sim}$.

\section{Lemma 4.40}

Die natürliche Transformation $\beta$ vermittelt einen Isomorphismus auf den Objekten der Kategorie I.

Beweis Die Aussage ist lokal in $S$, sei also $S$ ein affines Schema. Nach Satz 2.34 induziert $\beta$ für alle Objekte der Kategorie I einen Isomorphismus. Es folgt die Behauptung. 


\section{Lemma 4.41}

Die natürliche Transformation $\alpha$ vermittelt einen Isomorphismus von Objekten der Kategorie II.

Beweis Die Aussage ist lokal in $S$, sei also $S$ ein affines Schema. Sei $\left(M_{i}, M\right)$ ein Objekt der Kategorie II und sei $\mathcal{M}:=\bigoplus_{i \geq 0} M_{i}$. Nach [Har77], Kapitel II, Aufgabe 5.9, Seite 125 induziert $\alpha$ Isomorphismen

$$
M_{i} \cong\left(\Gamma_{*}\left(\mathcal{M}^{\sim}\right)\right)_{i}
$$

für alle $i \geq n_{0}$ und eine geeignete Zahl $n_{0} \in \mathbb{N}$.

Sei $a \in A$ mit $\operatorname{deg} a>0$. Nach Voraussetzung ist

$$
M_{i+1} / M_{i} \stackrel{a}{\longrightarrow} M_{i+1+\operatorname{deg} a} / M_{i+\operatorname{deg} a}
$$

ein Isomorphismus für alle $i \geq 0$. Wir erhalten

$$
\begin{aligned}
& M_{i+1} / M_{i} \cong M_{i+1+\operatorname{deg} a^{n_{0}}} / M_{i+\operatorname{deg} a^{n_{0}}} \\
& \cong\left(\Gamma_{*}\left(\mathcal{M}^{\sim}\right)\right)_{i+1+\operatorname{deg} a^{n_{0}}} /\left(\Gamma_{*}\left(\mathcal{M}^{\sim}\right)\right)_{i+\operatorname{deg} a^{n_{0}}} \\
& \cong\left(\Gamma_{*}\left(\mathcal{M}^{\sim}\right)\right)_{i+1} /\left(\Gamma_{*}\left(\mathcal{M}^{\sim}\right)\right)_{i}
\end{aligned}
$$

für alle $i \geq 0$. Die Behauptung folgt mit Hilfe einer Induktion nach $i \geq 0$.

Damit ist Theorem 4.3 vollständig bewiesen.

\subsection{Drinfelds Theorem, erweiterte Fassung}

Wir erhalten mit Hilfe von Bemerkung 4.38 die folgende Erweiterung von Theorem 4.3.

Theorem 4.42 (Drinfeld, [Dri86])

Die folgenden beiden Kategorien sind äquivalent:

I') Kategorie aufsteigender Familien von $\mathcal{O}_{X \times S^{-}}$Vektorbündeln

$$
\longrightarrow \mathscr{E}_{i} \longleftrightarrow \mathscr{E}_{i+1} \longleftrightarrow \mathscr{E}_{i+2}-\cdots
$$

von Rang $\frac{d}{\operatorname{deg}(\infty)}$ mit:

1) Für alle $i \in \mathbb{Z}$ ist $\mathscr{E}_{i}(\infty) \cong \mathscr{E}_{i+d}$.

2) Für alle $i \in \mathbb{Z}$ ist $\operatorname{pr}_{S *} \mathscr{E}_{i+1} / \mathscr{E}_{i}$ ein $\mathcal{O}_{S}$-Geradenbündel.

3) Es ist $H^{0}\left(X \times S, \mathscr{E}_{-1}\right)=H^{1}\left(X \times S, \mathscr{E}_{-1}\right)=0$. 
II') Kategorie der $\mathcal{O}_{S^{-}}$Moduln $\mathscr{M}$ mit einer aufsteigenden Filtrierung von $\mathcal{O}_{S}$-Moduln $\mathscr{M}_{i}$ und einem Ringhomomorphismus $A \longrightarrow \operatorname{End}_{\mathcal{O}_{S}}(\mathscr{M})$ mit

a) Es ist $\lim _{\longrightarrow} \mathscr{M}_{i}=\mathscr{M}$.

b) Für alle $i \in \mathbb{Z}$ sind die $\mathcal{O}_{S}-$ Moduln $\mathscr{M}_{i}$ Vektorbündel von Rang $\max (i+1,0)$. Die Moduln $\mathscr{M}_{i} / \mathscr{M}_{i-1}$ sind Vektorbündel von Rang 1 für $i \geq 0$, von Rang 0 für $i<0$.

c) Für alle $a \in A, a \neq 0$ ist $a \mathscr{M}_{i} \subseteq \mathscr{M}_{i+d \operatorname{deg}(a)}$ und die Abbildung

$$
\mathscr{M}_{i} / \mathscr{M}_{i-1} \stackrel{a \cdot}{\longrightarrow} \mathscr{M}_{i+d \operatorname{deg}(a)} / \mathscr{M}_{i-1+d \operatorname{deg}(a)}
$$

ist ein Isomorphismus für alle $i \geq 0$.

\section{Satz 4.43}

Sei $\mathscr{E}_{i}$ wie in Theorem 4.42. Dann ist für alle $i \in \mathbb{Z}$ das $\mathcal{O}_{X \times S^{-}}$Vektorbündel $\mathscr{E}_{i}$ von allgemeinem Typ und für jedes $s \in S$ gilt $\chi\left(\left(\mathscr{E}_{i}\right)_{s}\right)=i+1$.

Beweis Da $H^{1}\left(X \times S, \mathscr{E}_{-1}\right)=0$ gilt, ist die Voraussetzung von Folgerung 2.51 erfüllt. Die Kohomologie von $\mathscr{E}_{-1}$ ist also mit beliebigem Basiswechsel in $S$ verträglich. Insbesondere gilt

$$
0=H^{0}\left(X \times S, \mathscr{E}_{-1}\right) \otimes_{\mathcal{O}_{S}} k(s) \cong H^{0}\left(X \times s,\left(\mathscr{E}_{-1}\right)_{s}\right)
$$

und

$$
0=H^{1}\left(X \times S, \mathscr{E}_{-1}\right) \otimes_{\mathcal{O}_{S}} k(s) \cong H^{1}\left(X \times s,\left(\mathscr{E}_{-1}\right)_{s}\right)
$$

für alle $s \in S$. Nach Lemma 4.2,2) ist $\mathscr{E}_{-1}$ ein Vektorbündel von allgemeinem Typ.

Für alle $k \in \mathbb{Z}$ ist nach Lemma $4.2,1)$ auch $\mathscr{E}_{-1}(k \infty)=\mathscr{E}_{-1+k d}$ von allgemeinem Typ. Es reicht also, die Aussage für $0 \leq i<d-1$ zu zeigen. Für $k \geq 0$ folgt aus der Sequenz

$$
0 \longrightarrow \mathscr{E}_{-1+k d} \longrightarrow \mathscr{E}_{i+k d} \longrightarrow \mathscr{E}_{i+k d} / \mathscr{E}_{-1+k d} \longrightarrow 0
$$

$H^{1}\left(X \times s,\left(\mathscr{E}_{i+k d}\right)_{s}\right)=0$ für alle $s \in S$. Für $k<0$ folgt aus der Sequenz

$$
0 \longrightarrow \mathscr{E}_{i+k d} \longrightarrow \mathscr{E}_{-1+(k-1) d} \longrightarrow \mathscr{E}_{-1+(k-1) d} / \mathscr{E}_{i+k d} \longrightarrow 0
$$

$H^{0}\left(X \times s,\left(\mathscr{E}_{i+k d}\right)_{s}\right)=0$ für alle $s \in S$. Nach Definition ist folglich $\mathscr{E}_{i}$ von allgemeinem Typ.

Die Berechnung der Euler-Charakteristik ergibt sich aus $\chi\left(\left(\mathscr{E}_{-1}\right)_{s}\right)=0$, $\chi\left(\left(\mathscr{E}_{i} / \mathscr{E}_{i-1}\right)_{s}\right)=1$ und der Sequenz

$$
0 \longrightarrow\left(\mathscr{E}_{i-1}\right)_{s} \longrightarrow\left(\mathscr{E}_{i}\right)_{s} \longrightarrow\left(\mathscr{E}_{i} / \mathscr{E}_{i-1}\right)_{s} \longrightarrow 0
$$




\section{Beweis (von Theorem 4.42)}

Kategorie I' $\Longrightarrow$ Kategorie II' Nach Satz 4.43 sind für alle $i \in \mathbb{Z}$ die $\mathcal{O}_{X \times S^{-}}$Vektorbündel $\mathscr{E}_{i}$ von allgemeinem Typ und definieren nach Satz 4.3 und Bemerkung 4.38 die $\mathcal{O}_{S^{-}}$Vektorbündel $\mathscr{M}_{i}:=\operatorname{pr}_{S *} \mathscr{E}_{i}$. Nach Voraussetzung ist $\operatorname{pr}_{S *}\left(\mathscr{E}_{i} / \mathscr{E}_{i-1}\right)$ ein $\mathcal{O}_{S}$-Geradenbündel. Für $i \geq 0$ gilt

$$
\mathscr{M}_{i} / \mathscr{M}_{i-1} \cong \operatorname{pr}_{S *} \mathscr{E}_{i} / \operatorname{pr}_{S *} \mathscr{E}_{i-1} \cong \operatorname{pr}_{S *}\left(\mathscr{E}_{i} / \mathscr{E}_{i-1}\right)
$$

und für $i<0$ gilt

$$
\mathscr{M}_{i} \cong \operatorname{pr}_{S *} \mathscr{E}_{i}=0
$$

Theorem 4.3 liefert nun die Behauptung.

Kategorie II' $\Longrightarrow$ Kategorie I' Für jedes $l$ mit $-1 \leq l<d-1$ sind die $\mathcal{O}_{S^{-}}$Vektorbündel $\left(\mathscr{M}_{l+d i}\right)$ Objekte der Kategorie II. Die Anwendung von Theorem 4.3 liefert $\mathcal{O}_{X \times S^{-}}$Vektorbündel $\mathscr{E}_{l}$ von allgemeinem Typ. Für alle $i \in \mathbb{Z}$ definieren wir durch

$$
\mathscr{E}_{l+i}:=\mathscr{E}_{l}(i \infty)
$$

eine aufsteigende Familie von $\mathcal{O}_{X \times S^{-}}$Vektorbündeln. Sie erfüllt nach Konstruktion die Bedingung 1). Aufgrund dieser Eigenschaft reicht es, die Bedingung 2) für $i \geq 0 \mathrm{zu}$ zeigen. In diesem Fall gilt

$$
\operatorname{pr}_{S *}\left(\mathscr{E}_{i} / \mathscr{E}_{i-1}\right) \cong \mathscr{M}_{i} / \mathscr{M}_{i-1}
$$

und Bedingung 2) ist erfüllt.

Für alle $s \in S$ gilt $\chi\left(\left(\mathscr{E}_{-1}\right)_{s}\right)=0$. Nach Folgerung 4.13 und Theorem 2.45 ist $H^{0}\left(X \times S, \mathscr{E}_{-1}\right)=H^{1}\left(X \times S, \mathscr{E}_{-1}\right)=0$. Dies zeigt die Gültigkeit der Bedingung 3$)$.

Die Funktorialität der beiden Konstruktionen und die Äquivalenz der beiden Kategorien ergibt sich analog zu Abschnitt 4.5. 


\section{$5 \quad$ Elliptische Garben}

\subsection{Definition von elliptischen Garben}

Seien $X, S, \infty$ wie im letzten Abschnitt.

\section{Definition $\mathbf{5 . 1}$}

Eine elliptische Garbe $\left(\mathscr{E}_{i}, s_{i}, t_{i}\right)_{i \in \mathbb{Z}}$ besteht aus den folgenden Daten:

1) $\mathscr{E}_{i}$ : Vektorbündel von Rangd auf $X \times S$

2) $s_{i}: \mathscr{E}_{i} \longrightarrow \mathscr{E}_{i+1}$ : Injektive Morphismen von $\mathcal{O}_{X \times S}$-Modulgarben

3) $t_{i}:{ }^{\tau} \mathscr{E}_{i} \longrightarrow \mathscr{E}_{i+1}:$ Injektive Morphismen von $\mathcal{O}_{X \times S}$-Modulgarben mit:

a) Das Diagramm

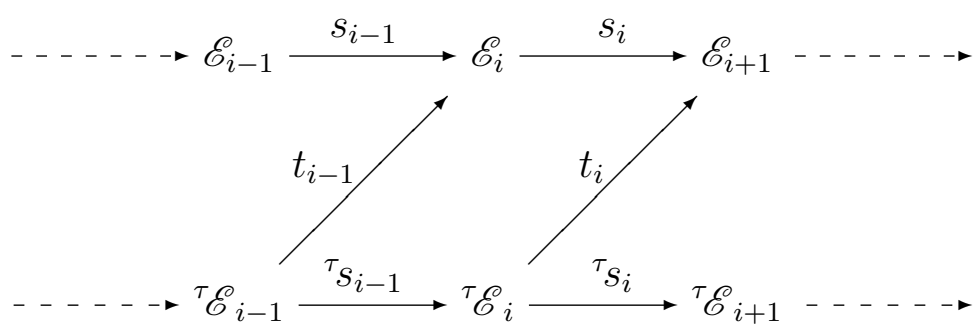

kommutiert.

b) Es gilt $\mathscr{E}_{i+d \operatorname{deg} \infty}=\mathscr{E}_{i} \otimes_{\mathcal{O}_{X \times S}} \operatorname{pr}_{X}^{*} \mathcal{O}_{X}(\infty)$.

c) Das Diagramm

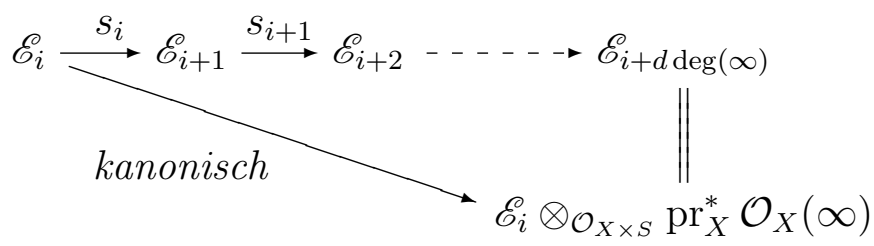

kommutiert.

d) Die $\mathcal{O}_{S}$-Modulgarbe $\left(\operatorname{pr}_{S}\right)_{*} \mathscr{E}_{i} / \mathscr{E}_{i-1}$ ist Geradenbündel auf $S$.

e) Die $\mathcal{O}_{S}$-Modulgarbe $\left(\operatorname{pr}_{S}\right)_{*} \mathscr{E}_{i} /{ }^{\tau} \mathscr{E}_{i-1}$ ist Geradenbündel auf $S$. 


\section{$5.2 \quad$ Eigenschaften}

Für $i \in \mathbb{Z}$ seien $\left(\mathscr{E}_{i}, s_{i}, t_{i}\right)$ Daten einer elliptischen Garbe.

\section{Bemerkung 5.2}

Äquivalent sind:

- Die Abbildungen $t_{i}:{ }^{\tau} \mathscr{E}_{i} /{ }^{\tau} \mathscr{O}_{i-1} \longrightarrow \mathscr{E}_{i+1} / \mathscr{E}_{i}$ sind Isomorphismen für alle $i \in \mathbb{Z}$.

- Für alle $x \in X \times S$ und $i \in \mathbb{Z}$ gilt

1) $\tau^{\tau} \mathscr{E}_{i, x} \cap \mathscr{E}_{i, x}={ }^{\tau} \mathscr{E}_{i-1, x}$

2) $\tau_{\mathscr{E}_{i, x}}+\mathscr{E}_{i, x}=\mathscr{E}_{i+1, x}$

\section{Folgerung 5.3}

Sei eine der äquivalenten Bedingungen aus Bemerkung 5.2 erfüllt. Dann gilt

$$
\mathscr{E}_{i, x} /^{\tau} \mathscr{E}_{i-1, x} \cong \mathscr{E}_{i+1, x} /^{\tau} \mathscr{E}_{i, x}
$$

für alle $i \in \mathbb{Z}$.

Beweis Es ist

$$
\begin{aligned}
\mathscr{E}_{i+1, x} /{ }^{\tau} \mathscr{E}_{i, x} & \cong\left({ }^{\tau} \mathscr{E}_{i, x}+\mathscr{E}_{i, x}\right) /^{\tau} \mathscr{E}_{i, x} \\
& \cong \mathscr{E}_{i, x} / \mathscr{E}_{i, x} \cap{ }^{\tau} \mathscr{E}_{i, x} \\
& \cong \mathscr{E}_{i, x} / \mathscr{\mathscr { E }}_{i-1, x} .
\end{aligned}
$$

\section{Folgerung 5.4}

In der Situation von Folgerung 5.3 ist für alle $i \in \mathbb{Z}$ die Sequenz

$$
0 \longrightarrow \tau_{\mathscr{E}_{i-1}} \stackrel{\left(t_{i-1},-\tau_{s_{i-1}}\right)}{\longrightarrow} \mathscr{E}_{i} \oplus^{\tau} \mathscr{E}_{i} \stackrel{\left(s_{i}+t_{i}\right)}{\longrightarrow} \mathscr{E}_{i+1} \longrightarrow 0
$$

exakt.

\section{Satz 5.5}

Äquivalent sind:

a) Für alle $i \in \mathbb{Z}$ ist $\operatorname{supp} \mathscr{E}_{i} / \mathscr{E}_{i-1} \cap \operatorname{supp} \mathscr{E}_{i} /{ }^{\tau} \mathscr{E}_{i-1}=\emptyset$.

b) Für alle $i \in \mathbb{Z}$ sind die Abbildungen $t_{i}:{ }^{\tau} \mathscr{E}_{i} /{ }^{\tau} \mathscr{E}_{i-1} \longrightarrow \mathscr{E}_{i+1} / \mathscr{E}_{i}$ Isomorphismen.

Wir zeigen zunächst a) impliziert b). 


\section{Lemma 5.6}

Für alle $i \in \mathbb{Z}$ sei $\operatorname{supp} \mathscr{E}_{i} / \mathscr{E}_{i-1} \cap \operatorname{supp} \mathscr{E}_{i} /{ }^{\tau} \mathscr{E}_{i-1}=\emptyset$. Dann sind die Abbildungen $t_{i}:{ }^{\tau} \mathscr{E}_{i} /{ }^{\tau} \mathscr{E}_{i-1} \longrightarrow \mathscr{E}_{i+1} / \mathscr{E}_{i}$ Isomorphismen für alle $i \in \mathbb{Z}$.

Beweis Da nach Voraussetzung für alle $x \in X \times S$ entweder $\left(s_{i}\right)_{x}$ oder $\left(t_{i}\right)_{x}$ ein Isomorphismus ist, folgt die Behauptung.

Nach Lemma 2.64 gilt $\operatorname{supp} \mathscr{E}_{i} / \mathscr{E}_{i-1} \subseteq \operatorname{spec} k(\infty) \times S$ für alle $i \in \mathbb{Z}$. Die Behauptung „b) impliziert a)" folgt aus dem folgenden Lemma.

\section{Lemma 5.7}

Für alle $i \in \mathbb{Z}$ ist $\operatorname{supp}\left(\mathscr{E}_{i+1} /{ }^{\tau} \mathscr{E}_{i}\right) \subseteq \operatorname{spec} A \times S$.

Beweis Die Aussage ist lokal in $S$. Sei deshalb $S=\operatorname{spec} R$ ein affines Schema. Sei $x \in \operatorname{spec} k(\infty) \otimes R$ und sei

$$
\iota: \operatorname{spec} k(\infty) \otimes R \longrightarrow \operatorname{spec} \mathcal{O}_{X, \infty} \otimes R
$$

die kanonische Einbettung. Sei $\mathfrak{p} \subseteq \mathcal{O}_{X, \infty} \otimes R$ das zu $\iota(x)$ gehörende Primideal mit $\mathfrak{p} \supseteq \mathfrak{m}_{X, \infty} \otimes R$. Es gilt für alle $i \in \mathbb{Z}$

$$
\begin{aligned}
\mathscr{E}_{i} & ={ }^{\tau} \mathscr{E}_{i-1}+\mathscr{E}_{i-1} \\
& ={ }^{\tau} \mathscr{E}_{i-1}+{ }^{\tau} \mathscr{E}_{i-2}+\mathscr{E}_{i-2}={ }^{\tau} \mathscr{E}_{i-1}+\mathscr{E}_{i-2} \\
& =\ldots \\
& ={ }^{\tau} \mathscr{E}_{i-1}+\mathscr{E}_{i-d}={ }^{\tau} \mathscr{E}_{i-1}+\mathscr{E}_{i} \otimes_{\mathcal{O}_{X \times S}} \mathcal{O}_{X \times S}(-\infty) .
\end{aligned}
$$

Lokalisierung nach $x$ ergibt

$$
\mathscr{E}_{i, x}={ }^{\tau} \mathscr{E}_{i-1, x}+\mathscr{E}_{i, x} \otimes_{\mathcal{O}_{X \times S, x}} \mathcal{O}_{X \times S}(-\infty)_{x} .
$$

Nach Definition von $\mathcal{O}_{X \times S}(-\infty)$ gilt

$$
\mathcal{O}_{X \times S}(-\infty)_{x}=\left(\mathfrak{m}_{X, \infty} \otimes R\right)_{\mathfrak{p}}
$$

Es folgt

$$
\mathscr{E}_{i, x}={ }^{\tau} \mathscr{E}_{i-1, x}+\mathscr{E}_{i, x} \otimes\left(\mathcal{O}_{X, \infty} \otimes R\right)_{\mathfrak{p}}\left(\mathfrak{m}_{X, \infty} \otimes R\right)_{\mathfrak{p}}
$$

Nach Voraussetzung ist $\mathscr{E}_{i, x}$ ein endlich erzeugter, projektiver $\left(\mathcal{O}_{X, \infty} \otimes R\right)_{\mathfrak{p}^{-}}$ Modul und es folgt

$$
\mathscr{E}_{i, x}={ }^{\tau} \mathscr{E}_{i-1, x}+\mathscr{E}_{i, x}\left(\mathfrak{m}_{X, \infty} \otimes R\right)_{\mathfrak{p}}
$$

Es ist $\left(\mathfrak{m}_{X, \infty} \otimes R\right)_{\mathfrak{p}} \subseteq \operatorname{Rad}\left(\left(\mathcal{O}_{X, \infty} \otimes R\right)_{\mathfrak{p}}\right)$ und mit dem Lemma von Nakayama folgt $\mathscr{E}_{i, x}=\tau_{\mathscr{E}_{i-1, x}}$ für alle $i \in \mathbb{Z}$. Es folgt die Behauptung.

\section{Folgerung $\mathbf{5 . 8}$}

Sei eine der beiden äquivalenten Bedingungen aus Satz 5.5 erfüllt. Dann gilt

$$
\operatorname{pr}_{S *} \mathscr{E}_{i} /^{\tau} \mathscr{E}_{i-1}=\operatorname{pr}_{S *}\left(\mathscr{E}_{i} / /\left.^{\tau} \mathscr{E}_{i-1}\right|_{\operatorname{spec} A \times S}\right)
$$

für alle $i \in \mathbb{Z}$. 


\subsection{Drinfeld-Moduln und elliptische Garben}

\section{Definition 5.9}

Sei $\mathscr{E} \ell \ell_{X}^{(d)}(S)$ die Kategorie der Elliptische Garben von Rang d mit den Eigenschaften:

1) Es ist $\chi\left(\mathscr{E}_{-1, s}\right)=0$ für alle $s \in S$.

2) Isomorphismen $t_{i}:{ }^{\tau} \mathscr{E}_{i} /{ }^{\tau} \mathscr{E}_{i-1} \longrightarrow \mathscr{E}_{i+1} / \mathscr{E}_{i}$ für alle $i \in \mathbb{Z}$.

\section{Theorem 5.10}

Es gibt eine Äquivalenz der Kategorien:

$$
\left\{\begin{array}{l}
\text { Drinfeld-Moduln von Rang } \\
d \text { über } S \text { in Standardform }
\end{array}\right\} \leftrightarrow\left\{\begin{array}{l}
\text { Elliptische Moduln der Ka- } \\
\text { tegorie } \mathscr{E} \ell \ell_{X}^{(d)}(S)
\end{array}\right\}
$$

Wir beweisen im Folgenden das Theorem 5.10.

\subsubsection{Elliptische Garben $\Longrightarrow$ Drinfeld-Moduln}

\section{Bemerkung 5.11}

Sei $S=\operatorname{spec} L$ ein Körper. Dann ist der Basiswechsel Frob $: L \longrightarrow L$ flach und es gilt $H^{i}\left(X \times S,{ }^{\tau} \mathscr{E}\right) \cong L \otimes_{L} H^{i}(X \times S, \mathscr{E})$ und $h^{i}(\mathscr{E})=h^{i}\left({ }^{\tau} \mathscr{E}\right)$ für $i=0,1$.

\section{Lemma 5.12}

Sei $\left(\mathscr{E}_{i}, s_{i}, t_{i}\right)$ ein Objekt der Kategorie $\mathscr{E}_{\ell} \ell_{X}^{(d)}(S)$ und sei $S=\operatorname{spec} L$ ein Körper. Sei $n_{0} \in \mathbb{Z}$ maximal mit $h^{0}\left(\mathscr{E}_{n_{0}}\right)=0$. Dann ist $n_{0}=-1$.

Beweis Wir zeigen zunächst, dass $H^{1}\left(X \times S,{ }^{\tau} \mathscr{E}_{n_{0}+k-1}\right)=0$ für alle $k \geq 1$ gilt. Es reicht, die Behauptung für $k=1$ zu zeigen, da $h^{1}\left(\mathscr{E}_{j}\right) \geq h^{1}\left(\mathscr{E}_{i}\right)$ für alle $i \geq j$ gilt. Sei

$$
\begin{aligned}
\mathscr{E}_{n_{0}+1}(X \times S) & \longleftrightarrow{ }^{\tau} \mathscr{E}_{n_{0}+1}(X \times S) \\
v & \longmapsto{ }^{\tau} v:=v \otimes 1
\end{aligned}
$$

für alle $i \in \mathbb{Z}$ die kanonische Abbildung. Sei $0 \neq v \in \mathscr{E}_{n_{0}+1}(X \times S)$ und sei $\tau v:=t\left({ }^{\tau} v\right)$.

Annahme: $\tau v \in \mathscr{E}_{n_{0}+1}(X \times S)$. Dann folgt

$$
\tau v \in \mathscr{E}_{n_{0}+1}(X \times S) \cap^{\tau} \mathscr{E}_{n_{0}+1}(X \times S)={ }^{\tau} \mathscr{E}_{n_{0}}(X \times S)=0
$$

im Widerspruch zu $\tau v \neq 0$. Da $h^{0}\left(\mathscr{E}_{n_{0}+2}\right) \leq 2$ gilt, folgt, dass die Abbildung

$$
\mathscr{E}_{n_{0}+1}(X \times S) \oplus^{\tau} \mathscr{E}_{n_{0}+1}(X \times S) \longrightarrow \mathscr{E}_{n_{0}+2}(X \times S)
$$


surjektiv ist und damit $H^{1}\left(X \times S,{ }^{\tau} \mathscr{E}_{n_{0}}\right)=0$ gilt (vgl. Folgerung 5.4).

Aus der exakten Sequenz

$$
0 \longrightarrow \mathscr{E}_{i-1} \longrightarrow \mathscr{E}_{i} \longrightarrow \mathscr{E}_{i} / \mathscr{E}_{i-1} \longrightarrow 0
$$

folgt $0 \leq h^{0}\left(\mathscr{E}_{i+1}\right)-h^{0}\left(\mathscr{E}_{i}\right) \leq 1$ für alle $i \in \mathbb{Z}(\operatorname{vgl}$. Beweis von Satz 4.9). Wir zeigen durch Induktion, dass $h^{0}\left(\mathscr{E}_{n_{0}+k}\right)=k$ gilt. Für $k=0,1$ ist dies die Definition von $n_{0}$.

Wir betrachten für $k \geq 1$ die exakte Sequenz aus Folgerung 5.4

$$
0 \longrightarrow \mathscr{E}_{n_{0}+k-1} \longrightarrow \mathscr{E}_{n_{0}+k} \oplus^{\tau} \mathscr{E}_{n_{0}+k} \longrightarrow \mathscr{E}_{n_{0}+k+1} \longrightarrow 0
$$

Übergang zu den globalen Schnitten ergibt, da $H^{1}\left(X \times S,{ }^{\tau} \mathscr{E}_{n_{0}+k-1}\right)=0$ gilt, die exakte Sequenz

$$
\begin{gathered}
0 \longrightarrow H^{0}\left(X \times S,{ }^{\tau} \mathscr{E}_{n_{0}+k-1}\right) \longrightarrow \\
\longrightarrow H^{0}\left(X \times S, \mathscr{E}_{n_{0}+k}(X \times S) \oplus H^{0}\left(X \times S,{ }^{\tau} \mathscr{E}_{n_{0}+k}\right) \longrightarrow\right. \\
\longrightarrow H^{0}\left(X \times S, \mathscr{E}_{n_{0}+k+1}\right) \longrightarrow 0
\end{gathered}
$$

für alle $k \geq 1$. Es folgt

$$
-h^{0}\left({ }^{\tau} \mathscr{E}_{n_{0}+k-1}\right)+h^{0}\left({ }^{\tau} \mathscr{E}_{n_{0}+k}\right)+h^{0}\left(\mathscr{E}_{n_{0}+k}\right)=h^{0}\left(\mathscr{E}_{n_{0}+k+1}\right)
$$

Also ist

$$
-(k-1)+2 k=k+1=h^{0}\left(\mathscr{E}_{n_{0}+k+1}\right) .
$$

Andererseits gilt $\chi\left(\mathscr{E}_{n_{0}+k}\right)=h^{0}\left(\mathscr{E}_{n_{0}+k}\right)=n_{0}+k+1$ für $k \geq 0$. Für $k \geq 1$ erhalten wir insgesamt

$$
k+1=h^{0}\left(\mathscr{E}_{n_{0}+k+1}\right)=n_{0}+k+2 \Longrightarrow n_{0}=-1 .
$$

\section{Folgerung 5.13}

Seien die Voraussetzungen wie in Lemma 5.12 erfüllt. Sei $0 \neq m_{0} \in H^{0}(X \times$ $\left.S, \mathscr{E}_{0}\right)$. Dann ist $m_{0}, \tau m_{0}, \ldots, \tau^{i} m_{0}$ eine $L$-Basis von $H^{0}\left(X \times S, \mathscr{E}_{i}\right)$ für alle $i \geq 0$.

Beweis Wir zeigen durch Induktion nach $i$ : Für alle $i \geq 0$ ist $\tau^{i} m_{0} \notin H^{0}(X \times$ $\left.S, \mathscr{E}_{i-1}\right)$.

Für $i=0$ ist $H^{0}\left(X \times S, \mathscr{E}_{-1}\right)=0$. Sei $i>0$. Annahme $\tau^{i} m_{0} \in H^{0}\left(X \times S, \mathscr{E}_{i-1}\right)$. Dann gilt

$$
\begin{gathered}
\tau^{i} m_{0} \in H^{0}\left(X \times S, \mathscr{E}_{i-1}\right) \cap H^{0}\left(X \times S,{ }^{\tau} \mathscr{E}_{i-1}\right) \\
\Longrightarrow{ }^{\tau}\left(\tau^{i-1} m_{0}\right) \in H^{0}\left(X \times S,{ }^{\tau} \mathscr{E}_{i-2}\right) .
\end{gathered}
$$


Andererseits ist die Abbildung

$H^{0}\left(X \times S, \mathscr{E}_{i-1}\right) / H^{0}\left(X \times S, \mathscr{E}_{i-2}\right) \longrightarrow H^{0}\left(X \times S,{ }^{\tau} \mathscr{E}_{i-1}\right) / H^{0}\left(X \times S,{ }^{\tau} \mathscr{E}_{i-2}\right)$

injektiv, also gilt $\tau^{i-1} m_{0} \in H^{0}\left(X \times S, \mathscr{E}_{i-2}\right)$ im Widerspruch zur Induktionsannahme.

\section{Satz 5.14}

Sei $\left(\mathscr{E}_{i}, s_{i}, t_{i}\right)$ ein Objekt der Kategorie $\mathscr{E}_{\ell} \ell_{X}^{(d)}(S)$. Dann ist $\mathscr{E}_{-1}$ ein Vektorbündel von allgemeinem Typ.

Beweis Aus Lemma 5.12 folgt

$$
H^{0}\left(X \times s,\left(\mathscr{E}_{-1}\right)_{s}\right)=0 \text { und } H^{1}\left(X \times s,\left(\mathscr{E}_{-1}\right)_{s}\right)=0
$$

für alle $s \in S$. Die Behauptung folgt aus Lemma 4.2,2).

Satz 5.15

Sei $\left(\mathscr{E}_{i}, s_{i}, t_{i}\right)$ ein Objekt der Kategorie $\mathscr{E} \ell \ell_{X}^{(d)}(S)$. Dann ist $\left(\mathscr{E}_{i}, s_{i}\right)$ ein Objekt der Kategorie I' (vgl. 4.62).

Beweis Die Bedingungen 1) und 2) sind nach Definition einer elliptischen Garbe bereits erfüllt. Bedingung 3) folgt aus Satz 5.15, Theorem 2.45, Satz 2.49, Satz 2.50 und Satz 4.15.

\section{Folgerung 5.16}

Für alle $i \in \mathbb{Z}$ ist

$$
\operatorname{pr}_{S *} \mathscr{E}_{i} /^{\tau} \mathscr{E}_{i-1} \cong \operatorname{pr}_{S *} \mathscr{E}_{0} /^{\tau} \mathscr{E}_{-1} \cong \operatorname{pr}_{S *} \mathscr{E}_{0}
$$

\section{Folgerung 5.17}

In der Situation von Folgerung 5.16 erhalten wir durch die $\mathcal{O}_{X}$-Modul-Struktur auf $\mathscr{E}_{i}$ nach Folgerung 5.16 eine A-Modul-Struktur auf $\mathrm{pr}_{S *} \mathscr{E}_{0}$. Wir erhalten also einen Ringhomomorphismus

$$
A \longrightarrow \operatorname{End}_{\mathcal{O}_{S}}\left(\operatorname{pr}_{S *} \mathscr{E}_{0}\right) \cong \mathcal{O}_{S}(S)
$$

\section{Definition 5.18}

Sei $\left(\mathscr{E}_{i}, s_{i}, t_{i}\right)$ ein Objekt der Kategorie $\mathscr{E} \ell \ell_{X}^{(d)}(S)$. Wir bezeichnen die Abbildung

$$
\text { char }: A \longrightarrow \operatorname{End}_{\mathcal{O}_{S}}\left(\operatorname{pr}_{S *} \mathscr{E}_{0}\right) \cong \mathcal{O}_{S}(S)
$$

aus Folgerung 5.17 als Charakteristik der elliptischen Garbe. 


\section{Folgerung 5.19}

Sei $S=\operatorname{spec} R$ ein affines Schema. Ist $\operatorname{pr}_{S_{*}} \mathscr{E}_{0}$ ein freier $R$-Modul, dann sind auch für alle $i \geq 0$ die $R$-Moduln $\operatorname{pr}_{S *} \mathscr{E}_{i}$ frei.

Beweis Sei $m_{0} \in H^{0}\left(X \times S, \mathscr{E}_{0}\right)$ ein nirgends verschwindender Schnitt, das heißt $0 \neq\left(m_{0}\right)_{s} \in H^{0}\left(X \times s,\left(\mathscr{E}_{0}\right)_{s}\right)$ für alle $s \in S$. Dann bilden die Elemente

$$
\left(m_{0}\right)_{s}, \tau\left(m_{0}\right)_{s}, \ldots \tau^{i}\left(m_{0}\right)_{s} \in H^{0}\left(X \times s,\left(\mathscr{E}_{i}\right)_{s}\right)
$$

nach Folgerung 5.13 eine Basis von $H^{0}\left(X \times s,\left(\mathscr{E}_{i}\right)_{s}\right)$ für alle $s \in S$ und definieren eine Trivialisierung des $R$-Moduls $\operatorname{pr}_{S *} \mathscr{E}_{i}$ für alle $i \geq 0$.

\section{Folgerung 5.20}

In der Situation von Folgerung 5.19 ist $H^{0}\left(U \times S, \mathscr{E}_{0}\right)$ ein freier $R\{\tau\}$-Modul von Rang 1.

Beweis Nach 2.70 ist $H^{0}\left(U \times S, \mathscr{E}_{0}\right) \cong \lim _{i} H^{0}\left(X \times S, \mathscr{E}_{i}\right)$. Wir definieren analog zu Lemma 5.12 eine $R\{\tau\}$-Modul-Struktur auf $H^{0}\left(U \times S, \mathscr{E}_{0}\right)$. Für $m \in H^{0}\left(X \times S, \mathscr{E}_{i}\right)$ setzen wir

$$
\tau m:=t_{i}\left({ }^{\tau} m\right) \in H^{0}\left(X \times S, \mathscr{E}_{i+1}\right) .
$$

Ist $m_{0} \in H^{0}\left(X \times S, \mathscr{E}_{0}\right)$ ein Basiselement, dann definiert die Abbildung

$$
\begin{aligned}
R\{\tau\} & \longrightarrow H^{0}\left(U \times S, \mathscr{E}_{0}\right) \\
1 & \longmapsto m_{0}
\end{aligned}
$$

nach Folgerung 5.19 einen Isomorphismus von $R\{\tau\}$-Moduln.

\subsubsection{Konstruktion eines Drinfeld-Modul}

\section{Satz 5.21}

Sei $\left(\mathscr{E}_{i}, s_{i}, t_{i}\right)$ ein Objekt der Kategorie $\mathscr{E}_{\ell} \ell_{X}^{(d)}(S)$ und sei die Voraussetzung von Lemma 5.19 erfüllt. Dann definiert $\left(\mathscr{E}_{i}, s_{i}, t_{i}\right)$ einen Drinfeld-Modul von Rang d in Standardform.

Beweis Wir wenden Theorem 4.42 an und erhalten projektive $R$-Moduln $\left(M, M_{i}\right)$ für alle $i \in \mathbb{Z}$, die der Bedingung von Kategorie II' genügen. Nach Folgerung 5.19 sind für alle $i \in \mathbb{Z}$ die $R$-Moduln $M_{i}$ frei von Rang $\max (0, i+$ 1). Folgerung 5.20 definiert nach Wahl eines Basiselements $m_{0} \in M_{0}$ einen Isomorphismus $M \cong R\{\tau\}$ von $R\{\tau\}$-Moduln. Wir definieren durch

$$
\begin{aligned}
& A \longrightarrow M \stackrel{\cong}{\longrightarrow} R\{\tau\} \\
& a \longmapsto a m_{0} \longrightarrow \varphi(a)
\end{aligned}
$$


eine $\mathbb{F}_{q}$-lineare Abbildung $\varphi: A \longrightarrow R\{\tau\} \operatorname{mit}^{\operatorname{deg}_{\tau}} \varphi(a) \leq d \operatorname{deg}(\infty) \operatorname{deg}(a)$ für alle $0 \neq a \in A$. Da für alle $i \in \mathbb{Z}$ die Abbildung $t_{i}$ nach Voraussetzung $\mathcal{O}_{X}$-linear ist, gilt

$$
a(\tau m)=\tau(a m)
$$

für alle $m \in M$. Es folgt $\varphi(a b)=\varphi(a) \varphi(b)$ und also ist $\varphi$ ein Ringhomomorphismus.

Weiterhin erhalten wir nach Definition der Kategorie II' für jedes Element $0 \neq a \in A$ einen Isomorphismus

$$
M_{0} \stackrel{a}{\longrightarrow} M_{d \operatorname{deg} \infty \operatorname{deg}(a)} / M_{d \operatorname{deg} \infty \operatorname{deg}(a)-1}
$$

Sei

$$
R\{\tau\}_{\leq n}:=\left\{f(\tau) \in R\{\tau\} \mid \operatorname{deg}_{\tau} f(\tau) \leq n\right\}
$$

für alle $n \in \mathbb{N}$. Der Isomorphismus $M \cong R\{\tau\}$ vermittelt nach Konstruktion einen $R$-linearen Isomorphismus

$$
\begin{aligned}
M_{d \operatorname{deg} \infty \operatorname{deg} a} / M_{d \operatorname{deg} \infty \operatorname{deg}(a)-1} & \cong R\{\tau\}_{\leq d \operatorname{deg}(\infty) \operatorname{deg} a} / R\{\tau\}_{\leq d \operatorname{deg}(\infty) \operatorname{deg} a-1} \\
& \cong R .
\end{aligned}
$$

Sei $\varphi(a):=\sum_{n=0}^{d \operatorname{deg}(\infty) \operatorname{deg}(a)} r_{n} \tau^{n}$. Wir erhalten einen $R$-linearen Isomorphismus

$$
\begin{aligned}
& M_{0} \stackrel{a}{\longrightarrow} R \\
& m_{0} \longmapsto r_{d \operatorname{deg} \infty \operatorname{deg} a} .
\end{aligned}
$$

Da $m_{0}$ den $R$-Modul $M_{0}$ erzeugt, ist folglich $r_{d \operatorname{deg} \infty \operatorname{deg} a}$ eine Einheit in $R$. Der konstruierte Drinfeld-Modul ist also von Rang $d$ und in Standardform. Die Charakteristik des Drinfeld-Moduls erhalten wir durch Komposition der Abbildung $\varphi$ mit der Abbildung $\partial: R\{\tau\} \longrightarrow R$.

\subsubsection{Drinfeld-Moduln $\Longrightarrow$ Elliptische Garben}

Die Daten 1), 2) und 3) einer elliptischen Garbe (vgl. 5.1) ergeben sich durch die Konstruktion in Abschnitt 3 und Bemerkung 3.19. Ebenso ergibt sich die Kommutativität des Diagramms in a) aus der Konstruktion.

zu b), c) Es ist $\left.\left(\operatorname{pr}_{X}^{*} \mathcal{O}_{X}(\infty)\right)\right|_{\operatorname{spec}(A) \times S} \cong \mathcal{O}_{\text {spec } A \times S}$ und die Behauptung ergibt sich aus der Konstruktion und Folgerung 3.17.

zu d) Nach Konstruktion ist $\operatorname{supp} \mathscr{E}_{i} / \mathscr{E}_{i-1} \subseteq \operatorname{spec} \mathcal{O}_{X, \infty} \times S$. Die Behauptung ergibt sich nun aus 2) in Beweis zu Satz 3.10 und Lemma 5.3. 
zu e) Folgerung 3.16 zeigt, dass $\operatorname{supp} \mathscr{E}_{i} /{ }^{\tau} \mathscr{E}_{i-1} \subseteq \operatorname{spec} A \times S$ gilt. Dann ergibt sich die Behauptung aus der Beziehung

$$
\operatorname{pr}_{S *} \mathscr{E}_{i} /^{\tau} \mathscr{E}_{i-1} \cong \operatorname{pr}_{S *}\left(\mathscr{E}_{i} /\left.^{\tau} \mathscr{E}_{i-1}\right|_{\text {spec } A \times S}\right) \cong \operatorname{pr}_{S *} \mathscr{E}_{0} \cong R
$$

für alle $i \in \mathbb{Z}$.

\subsection{Beliebiges Basisschema}

Wir konstruieren im Folgenden die Korrespondenz zwischen Drinfeld-Moduln und elliptischen Garben über einem beliebigem Basisschema $S$. Hierzu benötigen wir das folgende Lemma.

\section{Lemma 5.22}

Es gilt

$$
\operatorname{pr}_{S *}\left(\left.\mathscr{E}_{0}\right|_{\mathrm{spec} A \times S}\right) \cong \bigoplus_{n=0}^{\infty}\left(\operatorname{pr}_{S *} \mathscr{E}_{0}\right)^{\tau^{n}}
$$

Beweis Nach Satz 2.70 ist

$$
\operatorname{pr}_{S *}\left(\left.\mathscr{E}_{0}\right|_{\operatorname{spec} A \times S} \cong \underset{i \geq 0}{\lim _{i \geq 0}} \operatorname{pr}_{S *} \mathscr{E}_{i}\right.
$$

Für alle $i \in \mathbb{Z}$ gibt es nach Voraussetzung die exakte Sequenz

$$
0 \longrightarrow \mathscr{E}_{i-1} \longrightarrow \mathscr{E}_{i} \longrightarrow \mathscr{E}_{i} /^{\tau} \mathscr{E}_{i-1} \longrightarrow 0
$$

Die Anwendung des Funktors $\operatorname{pr}_{S *}$ auf die Sequenz ist für $i \geq 0$ exakt. Mit Hilfe der Einbettung

$$
s_{i-1} \circ \cdots \circ s_{0}: \mathscr{E}_{0} \longrightarrow \mathscr{E}_{i}
$$

und Folgerung 5.16 erhalten wir das Diagramm

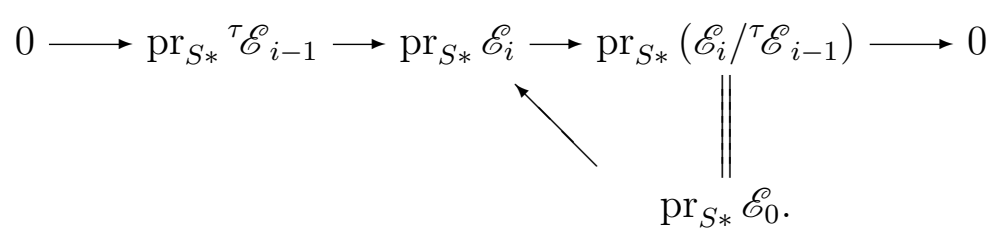

Die Sequenz spaltet also und wir erhalten für alle $i \geq 0$

$$
\operatorname{pr}_{S *} \mathscr{E}_{i} \cong \operatorname{pr}_{S *}{ }^{\tau} \mathscr{E}_{i-1} \oplus \operatorname{pr}_{S *} \mathscr{E}_{0}
$$

Nach Folgerung 2.51 gilt $\operatorname{pr}_{S *}{ }^{\tau} \mathscr{E}_{i-1} \cong \tau\left(\operatorname{pr}_{S *} \mathscr{E}_{i-1}\right)$. Der Übergang zum induktiven Limes liefert die Behauptung. 


\subsubsection{Drinfeld-Moduln $\Longrightarrow$ Elliptische Garben}

Sei $E=\left(\mathbb{G}_{a / \mathscr{L}}, e\right)$ ein Drinfeld-Modul über $S$ in Standardform. Wie in Abschnitt 3.8 beschrieben, konstruieren wir für alle $i \in \mathbb{Z}$ die $\mathcal{O}_{X \times S^{-}}$Vektorbündel

$$
\mathscr{E}_{i}:=\operatorname{Proj}\left(\bigoplus_{k=0}^{\infty} \bigoplus_{n=0}^{i+k d \operatorname{deg} \infty} \mathscr{L}^{q^{-n}}\right)
$$

Für alle $i \in \mathbb{Z}$ induzieren die kanonischen Einbettungen

$$
\bigoplus_{n=0}^{i+k d \operatorname{deg} \infty} \mathscr{L}^{-q^{n}} \longrightarrow \bigoplus_{n=0}^{i+1+k d \operatorname{deg} \infty} \mathscr{L}^{-q^{n}}
$$

homogene, $\mathcal{S}_{X} \otimes \mathcal{O}_{S}$-lineare Abbildungen

$$
\mathcal{M}_{i} \hookrightarrow \mathcal{M}_{i+1}
$$

von Grad 0 und damit $\mathcal{O}_{X \times S^{-}}$-lineare Abbildungen

$$
s_{i}: \mathscr{E}_{i} \longrightarrow \mathscr{E}_{i+1}
$$

Es gilt für alle $i \in \mathbb{Z}$

$$
\left(\bigoplus_{n=0}^{i+k d \operatorname{deg} \infty} \mathscr{L}^{-q^{n}}\right)^{\tau} \cong \bigoplus_{n=0}^{i+k d \operatorname{deg} \infty} \mathscr{L}^{-q^{n+1}} \cong \bigoplus_{n=1}^{i+1+k d \operatorname{deg} \infty} \mathscr{L}^{-q^{n}}
$$

Wir erhalten also durch die kanonischen Inklusionen

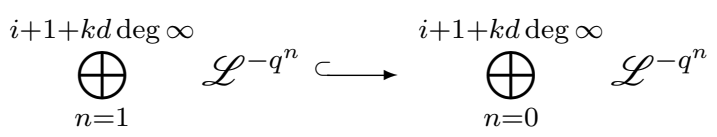

homogene, $\mathcal{S}_{X} \otimes \mathcal{O}_{S^{-}}$lineare Abbildungen

$$
\mathcal{M}_{i}^{\tau} \smile \mathcal{M}_{i+1}
$$

von Grad 0. Diese induzieren $\mathcal{O}_{X \times S^{-}}$lineare Abbildungen

$$
t_{i}:{ }^{\tau} \mathscr{E}_{i} \longrightarrow \mathscr{E}_{i+1}
$$

Für alle $j \in \mathbb{Z}$ induziert die kanonische Abbildung

$$
\mathcal{S}_{X}[1]_{j} \otimes_{\mathcal{S}_{X}} \bigoplus_{n=0}^{i+k d \operatorname{deg} \infty} \mathscr{L}^{-q^{n}} \longrightarrow \bigoplus_{n=0}^{i+k d \operatorname{deg} \infty+(j+1) d \operatorname{deg} \infty} \mathscr{L}^{-q^{n}}
$$


homogene, $\mathcal{S}_{X} \otimes \mathcal{O}_{S^{-}}$lineare Abbildungen

$$
\mathcal{S}_{X}[1] \otimes_{\mathcal{S}_{X}} \mathcal{M}_{i} \longrightarrow \mathcal{M}_{i+d \operatorname{deg} \infty}
$$

ebenfalls von Grad 0. Diese induzieren $\mathcal{O}_{X \times S^{-}}$lineare Abbildungen

$$
\mathcal{O}_{X \times S}(\infty) \otimes_{\mathcal{O}_{X \times S}} \mathscr{E}_{i} \longrightarrow \mathscr{E}_{i+d \operatorname{deg} \infty}
$$

Die Konstruktion stimmt lokal in $S$ mit der Konstruktion in Abschnitt 5.3.3 überein. Die Bedingungen an ein Objekt der Kategorie $\mathscr{E} \ell \ell_{X}^{(d)}(S)$ lassen sich lokal in $S$ testen und sind in diesem Fall bereits in Abschnitt 5.3.3 gezeigt.

\subsubsection{Elliptische Garben $\Longrightarrow$ Drinfeld-Moduln}

Sei $\left(\mathscr{E}_{i}, s_{i}, t_{i}\right)$ eine elliptische Garbe der Kategorie $\mathscr{E} \ell \ell_{X}^{(d)}(S)$. Sei $U:=\operatorname{spec} A$ $\subseteq X$. Nach Lemma 5.22 gilt

$$
\operatorname{pr}_{S *}\left(\left.\mathscr{E}_{0}\right|_{U \times S}\right) \cong \bigoplus_{i \geq 0}\left(\operatorname{pr}_{S *} \mathscr{E}_{0}\right)^{\tau^{n}}
$$

Sei

$$
\mathscr{L}^{-1}:=\operatorname{pr}_{S *} \mathscr{E}_{0}
$$

Dann gilt

$$
\left.\operatorname{pr}_{S *} \mathscr{E}_{0}\right|_{U \times S} \cong \bigoplus_{n \geq 0} \mathscr{L}^{-q^{n}} \cong \mathscr{H}_{0 m_{\mathbb{F}_{q}}}\left(\mathbb{G}_{a / \mathscr{L}}, \mathbb{G}_{a / \mathcal{O}_{S}}\right)
$$

Sei $a \in A$. Dann vermittelt $a$ eine $\mathcal{O}_{S^{-}}$lineare Abbildung

$$
\operatorname{pr}_{S *}\left(\left.\mathscr{E}_{0}\right|_{U \times S}\right) \stackrel{a}{\longrightarrow} \operatorname{pr}_{S *}\left(\left.\mathscr{E}_{0}\right|_{U \times S}\right)
$$

Wir erhalten das Diagramm

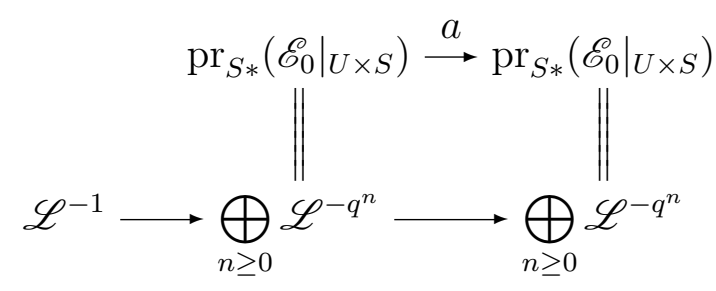

und dadurch eine Abbildung

$$
A \longrightarrow \mathscr{E} n d_{\mathbb{F}_{q}}\left(\mathbb{G}_{a / \mathscr{L}}\right) .
$$


Durch Nachschalten der kanonischen Projektion $\bigoplus_{n \geq 0} \mathscr{L}^{-q^{n}} \longrightarrow \mathscr{L}^{-1}$ erhalten wir eine Abbildung

$$
\operatorname{char}: A \longrightarrow \operatorname{End}_{\mathcal{O}_{S}}\left(\mathscr{L}^{-1}\right) \cong \mathcal{O}_{S}(S) .
$$

Dies ist die Charakteristik.

Die Konstruktion stimmt lokal in $S$ mit der Konstruktion in Abschnitt 5.3.1 überein. Die Bedingungen an einen Drinfeld-Modul lassen sich lokal in $S$ überprüfen und sind in diesem Fall bereits in Abschnitt 5.3.1 gezeigt.

\subsubsection{Drinfeld-Moduln $\Longrightarrow$ Kategorie II'}

In der in Abschnitt 5.4.1 durchgeführten Konstruktion einer elliptischen Garbe ist implizit die Konstruktion eines Objekts der Kategorie II' verborgen. Wir beschreiben sie im Folgenden.

Sei $E=\left(\mathbb{G}_{a / \mathscr{L}}, e\right)$ ein Drinfeld-Modul in Standardform. Sei $\mathscr{L}$ das zu $E$ gehörende $\mathcal{O}_{S^{-}}$Geradenbündel. Wir definieren

$$
\mathscr{M}_{i}:=\bigoplus_{n=0}^{i} \mathscr{L}^{-q^{n}}
$$

für alle $i \geq 0$. Für $i<0$ sei $\mathscr{M}_{i}=0$. Für alle $a \in A$ definiert die Abbildung

$$
e_{a}: \mathscr{L}^{-1} \longrightarrow \bigoplus_{n=0}^{d \operatorname{deg} \infty \operatorname{deg} a} \mathscr{L}^{q^{-n}}
$$

$\mathcal{O}_{S}$-lineare Morphismen

$$
\mathscr{M}_{i} \stackrel{a}{\longrightarrow} \mathscr{M}_{i+d \operatorname{deg} \infty \operatorname{deg} a} .
$$

Nach Definition eines Drinfeld-Moduls in Standardform ist für alle $0 \neq a \in A$ die Komposition der Abbildungen

$$
\mathscr{L}^{-1} \stackrel{e_{a}}{\longrightarrow} \bigoplus_{n=0}^{d \operatorname{deg} \infty \operatorname{deg} a} \mathscr{L}^{q^{-n}} \stackrel{\operatorname{pr}}{\longrightarrow} \mathscr{L}^{q^{-d \operatorname{deg} \infty \operatorname{deg} a}}
$$

ein Isomorphismus. Es folgt, dass für alle $i \geq 0$ auch die Abbildung

$$
\mathscr{M}_{i} / \mathscr{M}_{i-1} \stackrel{a}{\longrightarrow} \mathscr{M}_{i+d \operatorname{deg} \infty \operatorname{deg} a} / \mathscr{M}_{i-1+d \operatorname{deg} \infty \operatorname{deg} a}
$$

ein Isomorphismus ist. Wir erhalten also ein Objekt der Kategorie II'.

Seien $E=\left(\mathbb{G}_{a / \mathscr{L}}, e\right)$ und $E^{\prime}=\left(\mathbb{G}_{a / \mathscr{L}^{\prime}}, e^{\prime}\right)$ zwei Drinfeld-Moduln von Rang $d$ in Standardform. Sei $\varphi \in \operatorname{Hom}_{\mathbb{F}_{q}}\left(\mathbb{G}_{a / \mathscr{L}}, \mathbb{G}_{a / \mathscr{L}^{\prime}}\right)$ ein Morphismus von DrinfeldModuln. Nach Satz 3.1 gilt

$$
\varphi=\oplus_{m=0}^{\infty} \varphi_{m} \quad \text { mit } \quad \varphi_{m}: \mathscr{L}^{\prime-1} \longrightarrow \mathscr{L}^{-q^{m}} .
$$


Für alle $a \in A$ und alle $m \in \mathbb{N}$ erhalten wir das kommutative Diagramm

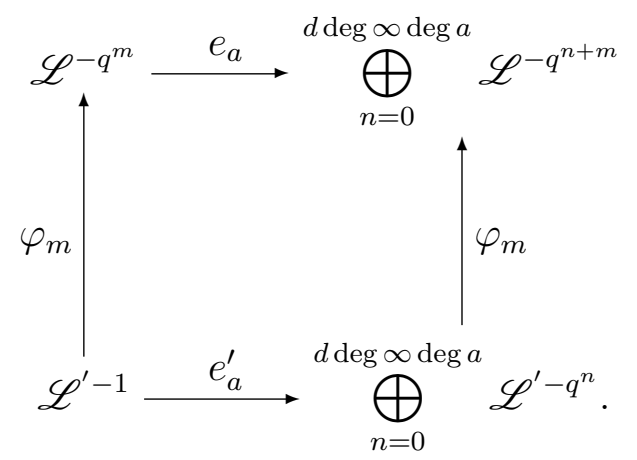

Sind $\left(\mathscr{M}_{i}, \mathscr{M}\right)$ und $\left(\mathscr{M}_{i}^{\prime}, \mathscr{M}^{\prime}\right)$ die zu den Drinfeld-Moduln $E$ und $E^{\prime}$ assoziierten Objekte der Kategorie II', dann erhalten wir folglich einen Morphismus

$$
\left(\mathscr{M}_{i}^{\prime}, \mathscr{M}^{\prime}\right) \stackrel{\varphi}{\longrightarrow}\left(\mathscr{M}_{i}, \mathscr{M}\right)
$$

von Objekten der Kategorie II'.

Wir erhalten also einen kontravarianten, treuen Funktor

$$
\left\{\begin{array}{l}
\text { Drinfeld-Moduln über } S \text { in } \\
\text { Standardform von Rang } d
\end{array}\right\} \leftrightarrow\{\text { Kategorie II' }\} \text {. }
$$

\subsubsection{Kategorienäquivalenz}

Wir kommen zum Schluss des Beweises von Theorem 5.10.

Durch die Konstruktion in den Abschnitten 5.4.1 und 5.4.2 erhalten wir Funktoren zwischen der Kategorie der Drinfeld-Modul von Rang $d$ in Standardform und der Kategorie $\mathscr{E} \ell \ell_{X}^{(d)}(S)$. Der Vergleich der Kategorie der DrinfeldModuln mit Kategorie II' (Abschnitt 5.4.3) und der Kategorie I' mit Kategorie II' (Theorem 4.42) zeigt die Funktorialität und Äquivalenz der beiden Kategorien. 


\section{Teilungspunkte und Level-Strukturen}

\subsection{Teilungspunkte und Level-Strukturen von Drinfeld-Moduln}

Sei $S$ ein Schema über dem endlichen Körper $\mathbb{F}_{q}$ und sei $E=\left(\mathbb{G}_{a / \mathscr{L}}, e\right)$ ein Drinfeld-Modul über $S$. Ist $S=\operatorname{spec} R$ ein affines Schema, dann bezeichen wir für alle $a \in A$ mit $e_{a} \in R\{\tau\}$ das Bild von $a$ bezüglich der Abbildung $e$. Jedes Element $f \in R\{\tau\}$ lässt sich als additives Polynom auffassen. Wir schreiben dann $f(X) \in R[X]$.

Sei $0 \neq I \subsetneq A$ ein Ideal.

\section{Definition 6.1}

Sei E $[I]$ der kontravariante Funktor auf der Kategorie der Schemata über $S$ mit Werten in der Kategorie der A/I-Moduln, gegeben durch die Zuordnung

$$
T / S \longmapsto\{x \in E(T) \mid I x=0\}=H_{A o m}(A / I, E(T))
$$

für alle Schemata $T / S$.

\section{Eigenschaften 6.2}

1) $E[I] \subseteq E$ ist ein abgeschlossenes Untergruppenschema. Ist $I=\left(a_{1}, \ldots a_{n}\right)$ für geeignete Elemente $a_{1}, \ldots, a_{n} \in A$, dann ist

$$
E[I]=\operatorname{Ker}\left(E \stackrel{e_{a_{1}, \ldots, e_{a_{n}}}^{\longrightarrow}}{\longrightarrow} E \times_{S} \cdots \times_{S} E\right) .
$$

Im affinen Fall $S=\operatorname{spec} R$ gilt

$$
E[I]=\operatorname{spec} R[X] /\left(e_{a_{1}}(X), \ldots, e_{a_{n}}(X)\right) .
$$

2) Sind $I, J$ teilerfremde Ideale in $A$, dann gilt

$$
E[I J] \cong E[I] \times{ }_{S} E[J]
$$

3) Das Gruppenschema $E[I]$ ist endlich und flach über $S$ von Rang $|A / I|^{d}$.

4) Ist $I$ teilerfremd zur Charakteristik des Drinfeld-Moduls $E$, dann ist $E[I]$ étale über $S$.

5) Das Gruppenschema $E[I]$ ist verträglich mit Basiswechsel, das heißt für jedes Schema $T / S$ gilt

$$
E[I] \times_{S} T \cong\left(E \times{ }_{S} T\right)[I]
$$


Beweis Siehe [Leh00], Kapitel 2, Satz 4.1, Seite 27 f.

Ist $S=\operatorname{spec} L$ ein Körper, dann ist die Charakteristik des Drinfeld-Moduls $(L, e)$ ein Primideal in $A$ und wir können die Höhe von $(L, e)$ definieren. Sie sei mit $h$ bezeichnet.

Im Falle eines algebraisch abgeschlossenen Körpers erhalten wir die folgende explizite Beschreibung der $I$-Teilungspunkte:

\section{Satz 6.3}

Sei $\mathfrak{p} \subseteq A$ ein Primideal und sei $I=\mathfrak{p}^{n}$ für ein $n>0$. Dann ist

$$
E\left[\mathfrak{p}^{n}\right](L) \cong \begin{cases}\left(\mathfrak{p}^{-n} / A\right)^{d} & \text { für } \mathfrak{p} \neq \text { char } E \\ \left(\mathfrak{p}^{-n} / A\right)^{d-h} & \text { für } \mathfrak{p}=\text { char } E .\end{cases}
$$

Beweis Siehe [Leh00], Kapitel 2, Korollar 2.4, Seite 24.

\section{Definition 6.4 ([Dri76])}

Sei $E=\left(\mathbb{G}_{a / \mathscr{L}}, e\right)$ ein Drinfeld-Modul von Rangd über $S$ und sei $0 \neq I \subsetneq A$ ein Ideal. Eine Level-I-Struktur ist eine A-lineare Abbildung

$$
\iota:\left(I^{-1} / A\right)^{d} \longrightarrow E(S)
$$

so dass für alle Primideale $0 \neq \mathfrak{p} \in V(I)$ eine Gleichheit der CartierDivisoren

$$
E[\mathfrak{p}]=\sum_{\alpha \in\left(\mathfrak{p}^{-1} / A\right)^{d}} \iota(\alpha)
$$

besteht.

\section{Bemerkung 6.5}

1) Sei $I$ teilerfremd zu char $E$. Dann ist eine Level- $I$-Struktur ein Isomorphismus von Gruppenschemata

$$
\left(I^{-1} / A\right)_{S}^{d} \simeq E[I]
$$

2) Ist $\iota$ eine Level- $I$-Struktur, dann gilt die Gleichheit der Cartier-Divisoren

$$
E[I]=\sum_{\alpha \in\left(I^{-1} / A\right)^{d}} \iota(\alpha) .
$$

Beweis Siehe [Leh00], Kapitel 2, Beispiel 4.3, Seite 29 und Kapitel 3, Satz 3.3, Seite 49. 


\section{Lemma 6.6}

Sei $R$ eine $\mathbb{F}_{q}$-Algebra. Sei $H \subset \mathbb{G}_{a / R}$ ein endliches, flaches Untergruppenschema von Rang $n$ über $R$. Dann gibt es ein eindeutig bestimmtes, normiertes, additives Polynom $h \in R[X]$ von Grad $n$ mit $H=V(h)$.

Beweis Siehe [Leh00], Kapitel 1, Lemma 3.3, Seite 9.

Sei $(R, e)$ ein Drinfeld-Modul von Rang $d$ über $R$ und sei $I \subsetneq A$ ein Ideal. Nach Lemma 6.6 gibt es ein additives Polynom $h_{I} \in R[X]$ von Rang $\left|I^{-1} / A\right|^{d}$ mit $E[I]=V\left(h_{I}\right)$. Die in der Definition der Level- $I$-Strukturen geforderte Gleichheit der Cartier-Divisoren bedeutet in diesem Fall die Gleichheit der Polynome

$$
h_{\mathfrak{p}}=\prod_{\alpha \in\left(\mathfrak{p}^{-1} / A\right)^{d}}(X-\iota(\alpha))
$$

für alle $\mathfrak{p} \supset I$.

\section{Satz 6.7}

Sei $R$ ein reduzierter Ring. Sei

$$
\iota:\left(I^{-1} / A\right)^{d} \longrightarrow E(R)
$$

eine A-lineare Abbildung. Ist $h_{I}=\prod_{\alpha \in\left(I^{-1} / A\right)^{d}}(X-\iota(\alpha))$, dann ist $\iota$ eine Level-I-Struktur.

\section{Bemerkung 6.8}

Ist char $E$ teilerfremd zu $I$, dann folgt die Behauptung bereits aus Bemerkung $6.5,1)$.

Beweis (von Satz 6.7) Wir zeigen die Behauptung zunächst für den Fall, dass $R=L$ ein Körper ist. Aus 6.2, 2) ergibt sich, dass es ausreicht, den Fall $I=\mathfrak{p}^{n}$ zu betrachten.

Nach Voraussetzung gilt $h_{\mathfrak{p}^{n}}=\prod_{\alpha \in\left(\mathfrak{p}^{-n} / A\right)^{d}}(X-\iota(\alpha))$. Nach Satz 6.3 gilt

$$
E\left[\mathfrak{p}^{n}\right](L) \cong\left(\mathfrak{p}^{-n} / A\right)^{d-h}
$$

Wir erhalten die exakte Sequenz von $A / \mathfrak{p}^{n}$-Moduln

$$
0 \longrightarrow \operatorname{ker} \iota \longrightarrow\left(\mathfrak{p}^{-n} / A\right)^{d} \stackrel{\iota}{\longrightarrow}\left(\mathfrak{p}^{-n} / A\right)^{d-h} \longrightarrow 0 .
$$

Folglich ist $\operatorname{ker} \iota \cong\left(\mathfrak{p}^{-n} / A\right)^{h}$ ein freier $A / \mathfrak{p}^{n}$-Modul. Für alle $n \in \mathbb{N}$ sei $k_{\mathfrak{p}^{n}}:=\left|\mathfrak{p}^{-n} / A\right|^{h}$, dann gilt

$$
h_{\mathfrak{p}^{n}}=\prod_{l \in \operatorname{Im} \iota}(X-l)^{k_{\mathfrak{p}} n} .
$$


Mit Hilfe der kanonischen Einbettung $\mathfrak{p}^{-1} / A \hookrightarrow \mathfrak{p}^{-n} / A$ definieren wir das Polynom

$$
\tilde{h}_{\mathfrak{p}}:=\prod_{\alpha \in\left(\mathfrak{p}^{-1} / A\right)^{d}}\left(X-\left.\iota\right|_{\left(\mathfrak{p}^{-1} / A\right)^{d}}(\alpha)\right) .
$$

Zu zeigen ist, dass $\tilde{h}_{\mathfrak{p}}=h_{\mathfrak{p}}$ gilt. Da Ker $\iota \cong\left(\mathfrak{p}^{-n} / A\right)^{h}$ gilt, folgt

$$
\left.\operatorname{Ker} \iota\right|_{\left(\mathfrak{p}^{-1} / A\right)^{d}} \cong\left(\mathfrak{p}^{-1} / A\right)^{h} \text {. }
$$

Also gilt

$$
\tilde{h}_{\mathfrak{p}}=\prod_{\left.l \in \operatorname{Im} \iota\right|_{(\mathfrak{p}-1 / A)^{d}}}(X-l)^{k_{\mathfrak{p}}} .
$$

Außerdem ist jedes $\left.l \in \operatorname{Im} \iota\right|_{\left(\mathfrak{p}^{-1} / A\right)^{d}}$ aufgrund der $A$-Linearität der Abbildung $\iota$ eine Nullstelle von $h_{\mathfrak{p}}$, da nach Definition $E[\mathfrak{p}]=V\left(h_{\mathfrak{p}}\right)$ gilt. Nach Satz 2.3 ist $E[\mathfrak{p}](L) \cong\left(\mathfrak{p}^{-1} / A\right)^{h}$. Das additive Polynom $h_{\mathfrak{p}}$ hat also genau $\left|\mathfrak{p}^{-1} / A\right|^{d-h}$ verschiedene Nullstellen und jede Nullstelle hat die Vielfachheit $k_{\mathfrak{p}}$. Es folgt die Behauptung.

Ist $R$ ein Integritätsring, so ergibt sich die Behauptung durch Übergang zum Quotientenkörper von $R$. Ist $R$ ein reduzierter Ring, dann können wir die Behauptung modulo aller Primideale $\mathfrak{P}$ von $R$ testen und erhalten die Behauptung zunächst für die Integritätsringe $R / \mathfrak{P}$. Im Fall eines reduzierten Ringes gilt jedoch $\bigcap_{\mathfrak{P} \in \text { spec } R}=(0)$ und es folgt wiederum die Behauptung.

\section{Bemerkung 6.9}

Im Falle eines beliebigen Ringes $R$ ist es dem Autor trotz einiger Bemühungen leider nicht gelungen, einen (elementaren) Beweis von Satz $6.7 \mathrm{zu}$ finden. Auch ein Gegenbeispiel ließ sich nicht konstruieren. Manche Autoren (z.B. [DH87], Definition 6.1) verwenden Satz 6.7 als Definition von Level- $I$ Strukturen. Eine Klärung des Problems wäre daher wünschenswert.

Vielleicht würde eine genaue Analyse des Beweises von [Leh00], Kapitel 3, Satz 3.3, einen Beweis des Satzes ermöglichen. Insbesondere müssten die benötigten Deformationsargumente auf den obigen Fall übertragen werden.

\section{$6.2 \quad F$-Garben}

Sei $S$ ein Schema über $\mathbb{F}_{q}$ und sei $\mathscr{E}$ ein $\mathcal{O}_{S^{-}}$Vektorbündel von Rang $n$. Mit $\mathscr{E}^{\vee}:=\mathscr{H}_{0} m_{\mathcal{O}_{S}}\left(\mathscr{E}, \mathcal{O}_{S}\right)$ sei das duale Vektorbündel bezeichnet.

Definition 6.10 ([Dri87])

Eine $\mathcal{O}_{S}$-lineare Abbildung

$$
\varphi: \mathscr{E} \tau \longrightarrow \mathscr{E}
$$


heißt Frobenius-Struktur auf $\mathscr{E}$. Wir nennen das Paar $(\mathscr{E}, \varphi)$ eine F-Garbe. Die Vektorbündel mit Frobenius-Struktur bilden in offensichtlicher Weise eine Kategorie. Sie heißt Kategorie der F-Garben über S.

\section{Bemerkung 6.11}

Der Frobenius-Homomorphismus $\mathscr{E}^{\vee} \longrightarrow \tau \mathscr{E}^{\vee}$ induziert einen Gruppenhomomorphismus

$$
\operatorname{Frob}^{g}: \mathbb{G}_{a / \mathscr{E} \vee} \longrightarrow \mathbb{G}_{a / \tau \mathscr{E} \vee} .
$$

\section{Definition 6.12 ([Dri87])}

Sei $(\mathscr{E}, \varphi)$ eine Frobenius-Struktur. Sie induziert einen Gruppenhomomorphismus

$$
\varphi^{g}: \mathbb{G}_{a / \mathscr{E} \vee} \longrightarrow \mathbb{G}_{a / \tau \mathscr{E}} \vee
$$

Sei

$$
\operatorname{Gr}(\mathscr{E}):=\operatorname{Ker}\left(\mathbb{G}_{a / \mathscr{E} \vee} \stackrel{\varphi^{g}-\mathrm{Frob}^{g}}{\longrightarrow} \mathbb{G}_{a / \mathscr{\tau}_{\mathscr{E}} \vee}\right)
$$

\section{Bemerkung 6.13}

Sei $(\mathscr{E}, \varphi)$ eine $F$-Garbe von Rang $n$.

1) Nach Konstruktion ist Gr ein Funktor von der Kategorie der F-Garben in die Kategorie der kommutativen Gruppenschemata über $S$.

2) Das Schema $\operatorname{Gr}(\mathscr{E})$ ist ein endliches, flaches Gruppenschema über $S$ von Rang $q^{n}$.

3) Das Schema $\operatorname{Gr}(\mathscr{E})$ ist genau dann étale über $S$, wenn $\varphi$ ein Isomorphismus ist.

4) Der Funktor Gr ist exakt, voll und treu.

Beweis Siehe [[Dri87]], Satz 2.1, Seite 110.

\section{Beispiel 6.14}

Sei $\mathscr{E} \cong \mathcal{O}_{S}^{\oplus n}$. Der kanonische Isomorphismus $\varphi:{ }^{\tau} \mathcal{O}_{S}^{\oplus n} \longrightarrow \mathcal{O}_{S}^{\oplus n}$ definiert eine Frobenius-Struktur auf $\mathcal{O}_{S}^{\oplus n}$. Für alle Schemata $T / S$ erhalten wir

$$
\begin{aligned}
\operatorname{Gr}\left(\mathcal{O}_{S}^{\oplus n}\right)(T) & =\left\{\alpha \in \operatorname{Hom}_{\mathcal{O}_{T}}\left(\mathcal{O}_{T}^{n}, \mathcal{O}_{T}\right) \mid \alpha^{q}=\alpha\right\} \\
& =\left(\operatorname{Hom}_{\mathbb{F}_{q}}\left(\mathbb{F}_{q}^{n}, \mathbb{F}_{q}\right)\right)_{S}(T)=\left(\mathbb{F}_{q}^{n \vee}\right)_{S}(T) .
\end{aligned}
$$

Es folgt $\operatorname{Gr}\left(\mathcal{O}_{S}^{\oplus n}\right) \cong\left(\mathbb{F}_{q}^{n \vee}\right)_{S}$. 


\section{Beispiel 6.15}

Sei $B$ eine endliche $\mathbb{F}_{q}$-Algebra von Rang $n$. Sei $\mathscr{E}:=\mathcal{O}_{S} \otimes B$. Wie im vorherigen Beispiel erhalten wir durch die Isomorphie ${ }^{\tau} \mathcal{O}_{S} \cong \mathcal{O}_{S}$ eine FrobeniusStruktur auf $\mathscr{E}$. Sei $T / S$ ein Schema. Es folgt

$$
\begin{aligned}
\operatorname{Gr}(\mathscr{E})(T) & =\left\{\alpha \in \operatorname{Hom}_{\mathcal{O}_{T}}\left(\mathcal{O}_{T} \otimes B, \mathcal{O}_{T}\right) \mid \alpha^{q}=\alpha\right\} \\
& =\operatorname{Hom}_{\mathbb{F}_{q}}\left(B, \mathcal{O}_{T}(T)\right) \\
& =\left(B^{\vee}\right)_{S}(T) .
\end{aligned}
$$

Die Funktoren $\operatorname{Gr}(\mathscr{E})$ und $\left(B^{\vee}\right)_{S}$ sind in natürlicher Weise $B$-Moduln und der Isomorphismus $\operatorname{Gr}(\mathscr{E}) \cong\left(B^{\vee}\right)_{S}$ ist $B$-linear.

\subsection{Teilungspunkte und Level-Strukturen elliptischer Garben}

Sei $\left(\mathscr{E}_{i}, s_{i}, t_{i}\right)$ eine elliptische Garbe der Kategorie $\mathscr{E} \ell \ell_{X}^{(d)}(S)$. Sei $0 \neq I \subsetneq A$ ein Ideal und sei $V(I) \subseteq U=\operatorname{spec} A$ das zugehörige abgeschlossene Unterschema von $U$.

Wir definieren

$$
\mathscr{F}:=\left.\operatorname{pr}_{S *} \mathscr{E}_{i}\right|_{U \times S}, \quad \mathscr{F}_{I}:=\left.\operatorname{pr}_{S *} \mathscr{E}_{i}\right|_{V(I) \times S}
$$

Die Definitionen sind unabhängig von der Wahl der Zahl $i$, da nach Voraussetzung $\left.\left.\mathscr{E}_{i}\right|_{U \times S} \cong \mathscr{E}_{i+1}\right|_{U \times S}$ für alle $i \in \mathbb{Z}$ gilt. Es folgt, dass $\mathscr{F}_{I}$ ein $\mathcal{O}_{S^{-}}$Vektorbündel von Rang $n:=|A / I|^{d}$ ist. Außerdem induziert die $\mathcal{O}_{X^{-}}$ Modul-Struktur auf $\mathscr{E}$ eine $A$-Modul-Struktur auf $\mathscr{F}_{I}$. Sei $\mathcal{O}_{S}\{\tau\}$ die $\mathcal{O}_{S^{-}}$ Algebren-Garbe definiert durch

$$
\mathcal{O}_{S}\{\tau\}(V):=\mathcal{O}_{S}(V)\{\tau\}
$$

für alle offenen Teilmengen $V \subseteq S$. Durch die $\mathcal{O}_{S^{-}}$lineare Abbildung $t:{ }^{\tau} \mathscr{F}_{I} \longrightarrow \mathscr{F}_{I}$ wird $\mathscr{F}_{I}$ zu einer $\varphi$-Garbe.

Es ist

$$
\mathscr{F}_{I} \cong \bigoplus_{n=0}^{\infty} \mathscr{L}^{-q^{n}} \otimes_{A} A / I
$$

Sei

$$
\bigoplus_{n=0}^{\infty} \mathscr{L}^{-q^{n}} \longrightarrow \bigoplus_{n=0}^{\infty} \mathscr{L}^{-q^{n}} \otimes_{A} A / I
$$

die kanonische Projektion.

Es folgt

$$
\operatorname{Gr}\left(\mathscr{F}_{I}\right)(T)=\operatorname{Hom}_{\mathcal{O}_{T}\{\tau\}}\left(\mathscr{F}_{T}, \mathcal{O}_{T}\right) \hookrightarrow \operatorname{Hom}_{\mathcal{O}_{T}\{\tau\}}\left(\bigoplus_{n=0}^{\infty} \mathscr{L}_{T}^{-q^{n}}, \mathcal{O}_{T}\right) \cong \mathscr{L}(T) .
$$


Wir erhalten einen kanonischen, A-linearen Gruppenhomomorphismus

$$
\operatorname{Gr}\left(\mathscr{F}_{I}\right) \hookrightarrow \mathbb{G}_{a / \mathscr{L}}
$$

Definition 6.16

Eine Level-I-Struktur einer elliptischen Garbe $\left(\mathscr{E}_{i}, s_{i}, t_{i}\right)$ ist eine A-lineare Abbildung

$$
\iota:(A / I)^{\vee d} \longrightarrow \mathscr{L}(S)
$$

so dass für alle Primideale $\mathfrak{p} \supseteq$ I eine Gleichheit der Divisoren

$$
\prod_{\alpha \in(A / \mathfrak{p})^{\vee d}}(X-\iota(\alpha))=\operatorname{Gr}\left(\mathscr{F}_{\mathfrak{p}}\right)
$$

besteht.

\section{Bemerkung 6.17}

Sind die Charakteristik der elliptischen Garbe und $V(I)$ disjunkt, dann ist Gr $\mathscr{F}_{I}$ ein étales Gruppenschema. Die Definition 6.16 ist in diesem Fall äquivalent mit der Angabe eines Isomorphismus

$$
\iota:\left((A / I)^{\vee d}\right)_{S} \longrightarrow \operatorname{Gr} \mathscr{F}_{I}
$$

(vgl. Bemerkung 6.5, 1)).

\section{Bemerkung 6.18}

Im Artikel [BS97] wird im Fall, dass die Charakteristik der Elliptischen Garbe und $V(I)$ disjunkt sind, eine Level- $I$-Struktur wie folgt definiert. Eine Level$I$-Struktur ist ein Isomorphismus $\iota$, so dass das Diagramm

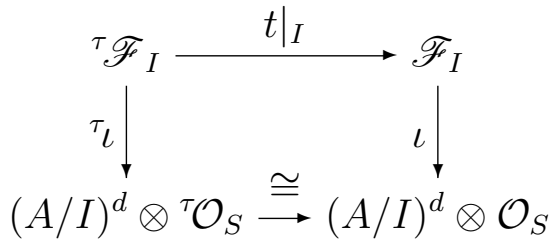

kommutiert. Die Abbildung $\iota$ ist also ein Isomorphismus der $F$-Garben $\left(\mathscr{F}_{I},\left.t\right|_{I}\right)$ und $\left((A / I)^{d} \otimes \mathcal{O}_{S}, \cong\right)$ (vgl. Beispiel 6.14). Wir zeigen die Äquivalenz der beiden Definitionen:

1) Die Anwendung des Funktors Gr liefert einen Isomorphismus

$$
\left((A / I)^{\vee d}\right)_{S} \longrightarrow \operatorname{Gr} \mathscr{F}_{I}
$$

(vgl. Beispiel 6.14).

2) Nach Bemerkung 6.13 ist der Funktor Gr voll und treu. Der Isomorphismus $\left((A / I)^{\vee d}\right)_{S} \longrightarrow$ Gr $\mathscr{F}_{I}$ induziert also einen Isomorphismus der zugehörigen $F$-Garben. 


\subsection{Vergleich der beiden Konzepte}

Wir zeigen im Folgenden, dass die Angabe einer Level-I-Struktur eines Drinfeld-Moduls äquivalent zur Angabe einer Level- $I$-Struktur einer elliptischen Garbe ist. Wir benötigen hierfür die beiden folgenden Lemmata:

\section{Lemma 6.19}

Sei $0 \neq I \subsetneq A$ ein Ideal. Dann sind die $A / I-M o d u l n A / I$ und $\operatorname{Hom}_{\mathbb{F}_{q}}\left(A / I, \mathbb{F}_{q}\right)$ isomorph.

Beweis Es reicht, die Behauptung für ein Ideal der Form $I=\mathfrak{p}^{e}$ zu zeigen. Sei also $e \in \mathbb{N}, 0 \neq \mathfrak{p}$ ein Primideal von $A$ und sei $\pi_{\mathfrak{p}} \in A$ ein uniformisierendes Element für das Primideal p. Dann bilden die Elemente

$$
\overline{1}, \bar{\pi}_{\mathfrak{p}}, \ldots, \bar{\pi}_{\mathfrak{p}}^{e-1} \in A / \mathfrak{p}^{e}
$$

eine $\mathbb{F}_{q}$-Basis von $A / \mathfrak{p}^{e}$. Sei $\psi \in \operatorname{Hom}_{\mathbb{F}_{q}}\left(A / \mathfrak{p}^{e}, \mathbb{F}_{q}\right)$ mit

$$
\psi(\overline{1})=1, \ldots, \psi\left(\bar{\pi}_{\mathfrak{p}}^{e-1}\right)=1 \text {. }
$$

Wir definieren die Abbildung

$$
\begin{array}{ccc}
\alpha: A / \mathfrak{p}^{e} & \longrightarrow & \operatorname{Hom}_{\mathbb{F}_{q}}\left(A / \mathfrak{p}^{e}, \mathbb{F}_{q}\right) \\
\bar{a} & \longmapsto & \bar{a} \psi .
\end{array}
$$

Nachrechnen zeigt, dass die Abbildung $A / \mathfrak{p}^{e}$-linear, wohldefiniert und injektiv ist. Als injektive Abbildung zwischen endlichen Mengen ist sie auch surjektiv und folglich ein Isomorphismus.

\section{Lemma 6.20}

Sei $0 \neq I \subsetneq A$ ein Ideal. Dann sind die $A / I$-Moduln $A / I$ und $I^{-1} / A$ isomorph.

Beweis Sei $I=\mathfrak{p}_{1}^{e_{1}} \cdots \mathfrak{p}_{s}^{e_{s}}$ die Primfaktorzerlegung des Ideals $I$. Es gibt ein Element $x \in A$ mit $v_{\mathfrak{p}_{1}}(x)=e_{1}, \ldots, v_{\mathfrak{p}_{s}}(x)=e_{s}$. Wir erhalten durch

$$
\begin{aligned}
I^{-1} / A & \longrightarrow A / I \\
a & \longmapsto x a
\end{aligned}
$$

eine $A / I$-lineare Abbildung. Die Abbildung ist ein Isomorphismus, da sie es nach Wahl des Elements $x$ an allen Primstellen von $A / I$ ist. 


\section{Satz 6.21}

Sei $\left(\mathscr{E}_{i}, s_{i}, t_{i}\right)$ eine elliptische Garbe der Kategorie $\mathscr{E}_{\ell} \ell_{X}^{(d)}(S)$ und sei $E=$ $\left(\mathbb{G}_{a / \mathscr{L}}, e\right)$ der assoziierte Drinfeld-Modul, über $S$ von Rang d. Sei $0 \neq I \subsetneq A$ ein Ideal und sei $\mathscr{F}_{I}$ die zugehörige F-Garbe. Dann ist die Angabe einer Level-I-Struktur für die elliptische Garbe $\left(\mathscr{E}_{i}, s_{i}, t_{i}\right)$ und die Angabe einer Level-I-Struktur für den Drinfeld-Modul E funktoriell äquivalent.

Beweis Nach Abschnitt 6.3 gilt für alle Schemata $T / S$

$$
\operatorname{Gr}\left(\mathscr{F}_{I}\right)(T)=\operatorname{Hom}_{\mathcal{O}_{T}\{\tau\}}\left(\mathscr{F}_{T}, \mathcal{O}_{T}\right) \hookrightarrow \operatorname{Hom}_{\mathcal{O}_{T}\{\tau\}}\left(\bigoplus_{n=0}^{\infty} \mathscr{L}_{T}^{-q^{n}}, \mathcal{O}_{T}\right) \cong \mathscr{L}(T) .
$$

Wir erhalten aus

$$
\operatorname{Hom}_{\mathcal{O}_{T}\{\tau\}}\left(\mathscr{F}_{T}, \mathcal{O}_{T}\right)=\left\{x \in \operatorname{Hom}_{\mathcal{O}_{T}\{\tau\}}\left(\bigoplus_{n=0}^{\infty} \mathscr{L}_{T}^{-q^{n}}, \mathcal{O}_{T}\right) \mid I x=0\right\}
$$

die Beschreibung

$$
\operatorname{Gr}\left(\mathscr{F}_{I}\right)(T)=\{x \in \mathscr{L}(T) \mid I x=0\} .
$$

Nach Definition der $I$-Teilungspunkte $E[I]$ eines Drinfeld-Moduls gilt

$$
E[I](T)=\{x \in \mathscr{L}(T) \mid I x=0\}
$$

und es gibt einen kanonischen Isomorphismus der Gruppenschemata

$$
\operatorname{Gr}\left(\mathscr{F}_{I}\right) \cong E[I]
$$

Nach Lemma 6.19 und Lemma 6.20 gibt es einen $A / I$-linearen Isomorphismus

$$
I^{-1} / A \stackrel{\sim}{\longrightarrow} \operatorname{Hom}_{\mathbb{F}_{q}}\left(A / I, \mathbb{F}_{q}\right) .
$$

Es folgt die Behauptung.

Wir folgern aus Satz 6.21 das Hauptresultat der Arbeit:

\section{Theorem 6.22}

Sei $0 \neq I \subsetneq A$ ein Ideal. Es gibt eine Äquivalenz der Kategorien:

$$
\left\{\begin{array}{l}
\text { Drinfeld-Moduln über } S \text { in } \\
\text { Standardform von Rang d } \\
\text { mit Level-I-Struktur }
\end{array}\right\} \leftrightarrow\left\{\begin{array}{l}
\text { Elliptische Moduln der Ka- } \\
\text { tegorie } \mathscr{E} \ell \ell_{X}^{(d)}(S) \text { mit Level- } \\
\text { I-Struktur }
\end{array}\right\}
$$

\section{Bemerkung 6.23}

Die Korrespondenz der beiden Kategorien hängt von der Wahl des Isomorphismus

$$
I^{-1} / A \stackrel{\sim}{\longrightarrow} \operatorname{Hom}_{\mathbb{F}_{q}}\left(A / I, \mathbb{F}_{q}\right)
$$

ab. 


\section{Ausblick}

\section{1 $\mathscr{D}$-elliptische Garben}

Wir stellen in diesem Abschnitt eine im Artikel [LRS93] beschriebene Verallgemeinerung der elliptischen Garben vor. Die verwendeten Notationen sind aus Kapitel 3 entnommen.

Sei $\eta \in X$ der generische Punkt der Kurve $X$. Sei $D$ eine zentrale, einfache $F$-Algebra und sei $\mathscr{D}_{X}$ eine lokal freie, kohärente $\mathcal{O}_{X}$-Algebra mit $\mathscr{D}_{X, \eta} \cong D$. Die Algebra $D$ zerfällt an fast allen Punkten $x \in X$. Wir nennen die endliche Menge der Punkte von $X$, an denen die Algebra nicht zerfällt, $\operatorname{Bad} \subseteq X$.

Sei $S$ ein Schema über $\mathbb{F}_{q}$ und sei $\mathscr{D}_{X \times S}:=\operatorname{pr}_{X}^{*} \mathscr{D}_{X}$.

Definition 7.1 ( $\mathscr{D}$-elliptische Garben, [LRS93])

Eine $\mathscr{D}$-elliptische Garbe $\left(\mathscr{E}_{i}, s_{i}, t_{i}\right)_{i \in \mathbb{Z}}$ besteht aus den folgenden Daten:

1) $\mathscr{E}_{i}$ : Vektorbündel von Rang $d^{2}$ auf $X \times S$ versehen mit einer Rechtsoperation der Algebrengarbe $\mathscr{D}_{X \times S}$, die mit der von links operierenden Strukturgarbe $\mathcal{O}_{X \times S}$ verträglich ist.

2) $s_{i}: \mathscr{E}_{i} \longrightarrow \mathscr{E}_{i+1}:$ Injektive Morphismen von $\mathcal{O}_{X \times S^{-}}$Modulgarben, die auch mit der $\mathscr{D}_{X \times S^{-}}$Operation verträglich sind.

3) $t_{i}: \tau_{\mathscr{E}_{i}} \longrightarrow \mathscr{E}_{i+1}:$ Injektive Morphismen von $\mathcal{O}_{X \times S}-$ Modulgarben, die ebenfalls mit der $\mathscr{D}_{X \times S^{-}}$Operation verträglich sind.

mit:

a) Das Diagramm

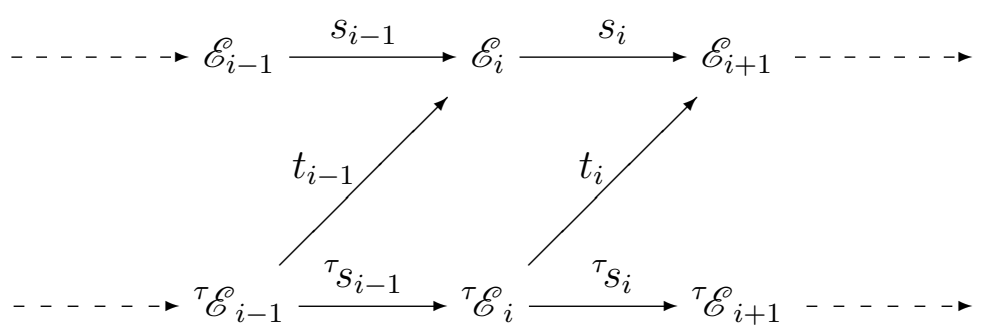

kommutiert.

b) Es gilt $\mathscr{E}_{i+d \operatorname{deg} \infty} \cong \mathscr{E}_{i} \otimes_{\mathcal{O}_{X \times S}} \operatorname{pr}_{X}^{*} \mathcal{O}_{X}(\infty)$.

c) Das Diagramm

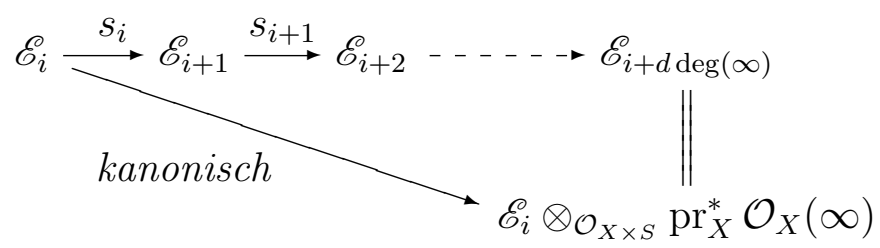


kommutiert.

d) Die $\mathcal{O}_{S}$-Modulgarbe $\left(\operatorname{pr}_{S}\right)_{*} \mathscr{E}_{i} / \mathscr{E}_{i-1}$ ist ein Vektorbündel von Rang d auf $S$.

e) Die $\mathcal{O}_{S}$-Modulgarbe $\left(\operatorname{pr}_{S}\right)_{*} \mathscr{E}_{i} / /^{\tau} \mathscr{E}_{i-1}$ ist ein Vektorbündel von Rang d auf $S$.

\section{Bemerkung 7.2}

Die Bedingungen b)und d) induzieren eine Abbildung (Pol-Morphismus)

$$
\mathrm{pol}: S \longrightarrow \operatorname{spec} k(\infty) \hookrightarrow X
$$

und die Bedingung e) induziert eine Abbildung (Zero-Morphismus)

$$
\text { zero }: S \longrightarrow X \text {. }
$$

\section{Bemerkung 7.3}

Analog zur Situation der elliptischen Garben sind für alle $i \in \mathbb{Z}$ die Abbildungen

$$
t_{i}:{ }^{\tau} \mathscr{E}_{i} /{ }^{\tau} \mathscr{E}_{i-1} \longrightarrow \mathscr{E}_{i+1} / \mathscr{E}_{i}
$$

Isomorphismen, falls $\infty \notin \operatorname{zero}(S)$ gilt.

\section{Bemerkung 7.4}

Die Autoren von [LRS93] fordern, um Probleme mit den Verzweigungspunkten der Algebra $D$ zu vermeiden, zusätzlich zu der Bedingung in Bemerkung 7.3, dass der Zero-Morphismus die Verzweigungspunkte nicht trifft, also insgesamt

$$
\operatorname{zero}(S) \subseteq X \backslash\{\infty \cup \operatorname{Bad}\}
$$

gilt.

\section{Beispiel 7.5}

Sei $D=\mathrm{M}_{d}(F)$ die $F$-Algebra der $d \times d$-Matrizen und sei $\mathscr{D}_{X \times S}:=\mathrm{M}_{d}\left(\mathcal{O}_{X \times S}\right)$. Dann erhalten wir mit Hilfe der Morita-Äquivalenz aus einer $\mathscr{D}$-elliptischen Garbe eine elliptische Garbe (vgl. [LRS93], 2.3, f).

\subsection{Level-Strukturen}

Mit Hilfe der F-Garben (vgl. Abschnitt 6.2 und 6.3) lassen sich auch für $\mathscr{D}$ elliptische Garbe Teilungspunkte definieren. Sei $0 \neq I \subsetneq A$ ein Ideal und sei $\left(\mathscr{E}_{i}, s_{i}, t_{i}\right)_{i \in \mathbb{Z}}$ eine $\mathscr{D}$-elliptische Garbe. Sei zero $(S) \subseteq X \backslash \infty$. Dann definieren wir analog zur Situation der elliptischen Garben

$$
\mathscr{F}:=\left.\operatorname{pr}_{S *} \mathscr{E}_{i}\right|_{U \times S}, \quad \mathscr{F}_{I}:=\left.\operatorname{pr}_{S *} \mathscr{E}_{i}\right|_{V(I) \times S}
$$

Die Definitionen sind nach Voraussetzung an den Zero-Morphismus unabhängig von der Wahl der Zahl $i$ und wir erhalten eine $F$-Garbe $\mathscr{F}_{I}$. 


\section{Definition 7.6 ([LRS93])}

Das Gruppenschema $\operatorname{Gr}\left(\mathscr{F}_{I}\right)$ heißt Schema der I-Teilungspunkte der $\mathscr{D}$ elliptischen Garbe.

Im Fall, dass $V(I) \subseteq X \backslash\{\operatorname{Bad} \cup \operatorname{zero}(S)\}$ gilt, definieren wir Level- $I$-Strukturen wie folgt:

Definition 7.7 ([LRS93])

Sei $\left.\mathscr{D}\right|_{I}:=\left.\mathscr{D}_{X}\right|_{V(I)}$. Eine Level-I-Struktur ist die Wahl eines Isomorphismus

$$
\iota:\left.\mathscr{F}_{I} \longrightarrow \mathscr{D}\right|_{I} \otimes \mathcal{O}_{S}
$$

von $\mathcal{O}_{V(I) \times S}$-Moduln, der mit der $\left.\mathscr{D}\right|_{I^{-}}$Operation verträglich ist und für den das Diagramm

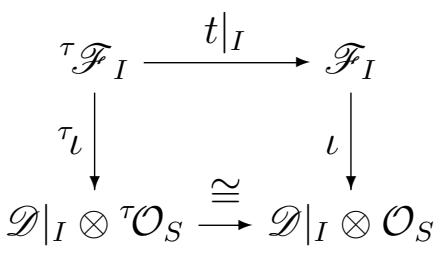

kommutiert.

Wir erhalten also einen Isomorpismus der $F$-Garben $\left(\mathscr{F}_{I},\left.t\right|_{I}\right)$ und $\left(\left.\mathscr{D}\right|_{I} \otimes\right.$ $\left.\mathcal{O}_{S}, \cong\right)$ der mit der $\left.\mathscr{D}\right|_{I^{-}}$Operation kompatibel ist (vgl. Beispiel 6.14).

\subsection{Level-Strukturen in der Charakteristik}

Es ist naheliegend, die Definition von Level-Stukturen aus Abschnitt 6.3 mit Hilfe der Morita-Äquivalenz auf den Fall $\mathscr{D}$-elliptischer Garben zu übertragen. Wir beschreiben im Folgenden kurz die Idee dieser Konstruktion.

Sei zero $(S) \subseteq X \backslash \infty$ und sei $0 \neq I \subsetneq A$ ein Ideal mit $V(I) \subseteq X \backslash\{\operatorname{Bad}\}$. Sei $\mathscr{L}:=\operatorname{pr}_{S *} \mathscr{E}_{0}$. Wie in Theorem 5.10 normieren wir die $\mathscr{D}$-elliptische Garbe so, dass $\mathscr{L}$ ein $\mathcal{O}_{S^{-}}$Vektorbündel von Rang $d$ ist.

Nach Abschnitt 6.3 erhalten wir für die F-Garbe $\mathscr{F}_{I}$ und alle Schemata $T / S$ die kanonische Abbildung

$$
\operatorname{Gr}\left(\mathscr{F}_{I}\right)(T)=\operatorname{Hom}_{\mathcal{O}_{T}\{\tau\}}\left(\mathscr{F}_{T}, \mathcal{O}_{T}\right) \hookrightarrow \operatorname{Hom}_{\mathcal{O}_{T}\{\tau\}}\left(\bigoplus_{n=0}^{\infty} \mathscr{L}_{T}^{-q^{n}}, \mathcal{O}_{T}\right) \cong \mathscr{L}(T)
$$

Sie ist nach Konstruktion auch $\left.\mathscr{D}\right|_{I}$-linear. Wir erhalten folglich einen kanonischen, $\left.\mathscr{D}\right|_{I^{-}}$linearen Gruppenhomomorphismus

$$
\operatorname{Gr}\left(\mathscr{F}_{I}\right) \smile \mathbb{G}_{a / \mathscr{L}} .
$$


Da $V(I)$ keinen der Verzweigungspunkte der Algebra $D$ trifft, können wir obige Abbildung mit Morita-Äquivalenz zur Situation im Fall der elliptischen Garben absteigen lassen und die für diesen Fall definierten Level- $I$ Strukturen auf den Fall $\mathscr{D}$-elliptischer Moduln übertragen. 


\section{Literaturverzeichnis}

[Bou98] Bourbaki, N.: Commutative Algebra. Springer, 1998.

[BS97] Blum, A. und U. Stuhler: Drinfeld Modules and Elliptic Sheaves. Springer. Lect. Notes Math., 1649:110-188, 1997.

[DH87] Deligne, P. und D. Husemöller: Survey of Drinfeld Modules. Contemporary Mathematics, 67:25-91, 1987.

[Dri76] Drinfeld, V. G.: Elliptic modules. Math. USSR Sbornic, 23:561592, 1976.

[Dri77] Drinfeld, V. G.: Elliptic modules II. Math. USSR Sbornic, 31:159-170, 1977.

[Dri86] Drinfeld, V. G.: Commutative subrings of certain noncommutative rings. Functional Analysis and its Application, 21:107-122, 1986.

[Dri87] Drinfeld, V. G.: Varieties of Modules of F-Sheaves. Functional Analysis and its Application, 21:107-122, 1987.

[Dri03] Drinfeld, V. G.: Infinite-Dimensional Vector Bundles in Algebraic Geometry, 2003.

[Eis94] Eisenbud, D.: Commutative Algebra with a View Toward Algebraic Geometry. Springer, 1994.

[GD61] Grothendieck, A. und J. Dieudonné: Éléments de Géométrie algébrique. Publ. Math. IHÉS, 8, 1961. EGA II.

[GD66] Grothendieck, A. und J. Dieudonné: Éléments de Géométrie algébrique. Publ. Math. IHÉS, 28:5-255, 1966. EGA IV.

[Gos98] Goss, D.: Basic Structures of Function Field Arithmetic. Springer, 1998.

[Har] Harder, G.: Lectures on Algebraic Geometry.

[Har77] Hartshorne, R.: Algebraic Geometry. Springer, 1977.

[Kap58] Kaplansky, I.: Projective modules. Annals of Math., 68, 1958. 
[Koc00] KocH, H.: Number Theory, Algebraic Numbers and Functions, Band 24 der Reihe Graduate Studies in Mathematics. American Mathematical Society, 2000.

[Leh00] Lenmkuhl, T.: Compactification of the Drinfeld modular surfaces. Habilitationsschrift, 2000.

[Liu02] LiU, Q.: Algebraic Geometry and Arithmetic Curves. Oxford University Press, 2002.

[LRS93] Laumon, G., M. Rappoport und U. Stuhler: D-elliptic sheaves and the Langlands correspondence. Invent. Math., 113:217-338, 1993.

[Mac94] Mac Lane, S.: Homology. Springer, 1994.

[Mat86] Matsumura, H.: Commutative ring theory. Cambrige University Press, 1986.

[Neu92] Neukirch, J.: Algebraische Zahlentheorie. Springer, 1992.

[RG71] Raynaud, M. und L. Gruson: Critères de platitude et de projectivité. Inventiones mathematicae, 13:1-89, 1971.

[Thi01] Tнімм, M.: Vektorbündel auf der nichtkommutativen projektiven Geraden. Diplomarbeit, Mathematisches Institut Göttingen, 2001.

[Wat79] Waterhouse, W. C.: Introduction to Affine Group Schemes. Springer, 1979.

[Wei94] Weibel, C. A.: An introduction to homological algebra. Cambridge University Press, 1994.

[Wie98] Wiedmann, S.: Hochschild-Homologie und Drinfeld-Moduln. Diplomarbeit, Mathematisches Institut Göttingen, 1998. 


\title{
Lebenslauf
}

\author{
19.01.1968 Geboren in Ochsenhausen, Deutschland \\ 1974 - 1977 Besuch der Grundschule Hürbel \\ 1977 - 1978 Besuch der Grund- und Hauptschule Gutenzell \\ 1978 - 1987 Besuch des Gymnasiums Ochsenhausen \\ $1987 \quad$ Abitur \\ 1987 - 1989 Zivildienst \\ 1989 - 1990 Studium der Physik \\ an der Georg-August-Universität Göttingen \\ 1990 - 1998 Studium der Mathematik \\ an der Georg-August-Universität Göttingen \\ 1998 Diplom in Mathematik \\ seit 1998 Wissenschaftlicher Mitarbeiter am Mathematischen \\ Institut der Georg-August-Universität Göttingen
}

